\title{
OPTIMAL CONTROL MODEL FOR THE TRANSMISSION DYNAMICS OF MALARIA-PNEUMONIA CO-INFECTION WITH MASS ACTION INCIDENCE
}

\author{
${ }^{1 *}$ ADENIYI Michael Olaniyi \\ Department of Mathematics and Statistics \\ Lagos State Polytechnic, Ikorodu \\ Lagos State, Nigeria \\ Email: adeniyi.m@mylaspotech.edu.ng \\ ${ }^{2}$ OLUYO Temitayo Olabisi
}

Department of Pure and Applied Mathematics Ladoke Akintola University of Technology

Ogbomoso, Oyo State Nigeria

Email: tooluyo@lautech.edu.ng

\begin{abstract}
Malaria and Pneumonia are leading causes of serious illness in children and adults worldwide with their death rate and prevalence on the rise. Such alarming statistics may retard the milestones so far achieved in meeting the Millennium Development Goals 4 and 6 whose targets are to improve child survival and reverse the high prevalence of diseases such as pneumonia and malaria respectively. Two sub-models of malaria-pneumonia co-infection namely malaria model and pneumonia model were considered first and then followed by the full malariapneumonia co-infection model. The malaria model, pneumonia model and co-infection model basic reproduction numbers denoted by $R_{m}, R_{p}$ and $R_{m p}$ respectively was obtained using the Next Generation Matrix method. The model disease free equilibrium's local and global stability was analysed using Descartes' Rule of signs and Comparison method. The bifurcation analysis for the malaria, pneumonia and co-infection models was studied using the Centre Manifold Theory. The sensitivity indices of the model basic reproduction numbers $R_{m}, R_{p}$ and $R_{m p}$ to the parameters in the models were calculated. Optimal control theory was applied using the Pontryagins' Maximum Principle to investigate optimal strategies for controlling the spread of malaria, pneumonia and coinfection models using insecticide treated bed nets $\left(u_{1}(t)\right)$, spraying of mosquitoes insecticides $\left(u_{2}(t)\right)$, sanitation $\left(u_{3}(t)\right)$, vaccination $\left(u_{4}(t)\right)$, anti-malaria drugs $\left(u_{5}(t)\right)$, anti-pneumonia drugs $\left(u_{6}(t)\right)$, both anti-malaria drugs and anti-pneumonia drugs $\left(u_{7}(t)\right)$ as the system time control variables. Numerical simulations using a set of parameter values were provided to validate the analytical results.
\end{abstract}

Keyword: Malaria, Mass Action, Malaria-Pneumonia, Optimal Control, Pneumonia

\subsection{INTRODUCTION}

Pneumonia and Malaria are two of the most deadly diseases of our time. The geographic overlap of these diseases in sub-Saharan Africa facilitates their co-infection. Although the consequences of the co-infection with pneumonia and malaria parasites are not fully understood in literature, available evidence now suggests that the infections act synergistically and their combination results in worse outcomes which poses a major public health concern. Malaria in an individual is an illness or disease that is caused by the parasites of the genus Plasmodium (Phylum Apicomplexa) in the blood or tissues [22]. The parasitic disease malaria is transmitted to the human through a biting from an infected female Anopheles mosquito [3]. The female Anopheles mosquito gets infected when it takes a blood meal from a person carrying the malaria parasite. There are four species of the plasmodium parasites, namely Plasmodium falciparum, Plasmodium ovale, Plasmodium vivax and Plasmodium malariae, of the four species, Plasmodium Falciparum is the most virulent, lethal and responsible for the majority of morbidity 
and mortality due to malaria [10]. Evidence of human infections with several species of Plasmodium from higher apes has been documented except for $P$. knowlesi - a zoonotic species that causes malaria in macaques these are mostly of limited public health importance [14]. Many children living in malaria-endemic areas are frequently exposed to other diseases such as pneumonia. Children have a higher risk of developing pneumonia if they have weakened immune system. A weak immune system may be as a result of prolonged malaria exposure, malnutrition among other factors. Statistics has shown that of all children out patients suffering from respiratory complications $25 \%$ of the cases are confirmed to be pneumonia [24].

Pneumonia is one of the forgotten killer diseases [37]. It is one of the major causes of children's' death in Africa and it kills more children per year than any other illness in the world [37]. The death rates are around 2 million children worldwide; every year [37]. Pneumonia is an inflammatory condition of the lungs; it is an airborne disease acquired through inhalation or aspiration of pulmonary pathogenic organisms into a lung segment or lobe [26]. Pneumonia could be caused by bacterial, virus, fungi and parasites [26]. The parasites infections usually enter the body through the skin or the mouth and progresses through the body to the lungs, usually through the blood [32]. Prevention of diseases such as pneumonia includes vaccination, environmental measures and appropriate treatment of other health problems [32].

Malaria and Pneumonia account for about $8 \%$ and 19\% of annual deaths in children [21]. The combination of both diseases contributes about $40 \%$ of the deaths in children below five years of age in sub-Saharan Africa [21]. Over six million new cases of pneumonia are estimated annually in Nigeria while childhood fevers presumed to be malaria account for $30 \%$ of all childhood deaths [31]. The WHO/UNICEF guidelines defines "malaria" as the presence or history of fever and symptoms that also occur in children with pneumonia, while "pneumonia" includes history of cough or difficulty in breathing in the presence of increased respiratory rate according to age and symptoms that may also indicate malaria [17]. Children who have malaria-pneumonia symptom overlap are given dual integrated management of childhood illness (IMCI) classifications and are treated with both antimalarial and antibiotics [19]. In Eastern Africa, the extent of the overlap has been documented but not in routine IMCI practice at the health centre level in Nigeria [22].

Mathematical models has been widely used to study and explains the transmission dynamics of the spread of malaria following the WHO position statement [36] that it is important to carry out modelling studies to determine the impact of various combinations of control strategies on the transmission dynamics of malaria. Series of studies have been done to quantify the impact of malaria infection in humans ([8], [18] and [20]). Many of these studies discusses only the transmission of the malaria disease in human and the vector populations, however, recent studies by Chiyaka et al.[13] focused on a deterministic system of differential equations involving two latent periods in the non-constant host and vector populations in order to analytically assess the potential impact of personal protection, treatment and possible vaccination strategies on the transmission dynamics of malaria. Blayneh et al. [7] formulated a time dependent model to consider the effects of prevention and treatment on malaria, while in similar fashion, Okosun et al. [28] studied a time dependent model on the possible impact of vaccination with treatment strategies in controlling the spread of malaria in a model that incorporate treatment and vaccination with waning immunity. Agusto et al. [2] studied a deterministic system of differential equations for the transmission of malaria and thereafter considered the optimal control strategies to investigate optimal strategies for controlling the spread of malaria disease using treatment, insecticide treated bed nets and spraying of mosquito insecticide as the system control variables. The possible impact of using combinations of the three controls either one at a time or two at a time on the spread of the disease was also examined in their study. 
Many mathematical model already exist describing malaria infection or pneumonia infection but the best control for malaria infection, pneumonia infection and the co-infection of both diseases still remain a subject of debate. Previous mathematical models have considered treatment, sleeping under mosquito treated bedside nets (MTBN) and the use of insecticide coil as controls. However, these have their limitations. The insecticides used for treating bedside nets is lethal to the mosquitoes, other insects and also repels the mosquitoes, thus reducing the number of mosquitoes who attempt to feed on people in the sleeping areas with the nets [2]. However, the mosquitoes can still feed on humans outside these protective areas, hence; Agusto et al [2] included the spraying of insecticides in their model. The latter control by [2] will be more effective in a closed area, hence, the inclusion of sanitation as control in the present work. The preventive and treatment controls for pneumonia includes vaccination, environmental measures and appropriate treatment of other health problems [32]. It is believed that, if appropriate preventive measures were instituted globally, mortality among children could be reduced by 400,000; and, if proper treatment were universally available, childhood deaths could be decreased by another $600,000[38]$.

The rest of the paper are organised in the following way: The mathematical formulation for the full co-infection model was presented in section 2, section 3 focused on the analysis of the sub models namely: malaria only model and pneumonia only model, this was later followed by the analysis of the full model on malaria-pneumonia co-infection. The optimal control analysis for the malaria only, pneumonia only and the co-infection model was considered in section 4 while the numerical simulations and the discussion of results was presented in section 5 .

\subsection{MATHEMATICAL FORMULATION}

Lawi et al [24] studied the following model on malaria-pneumonia co-infection with standard incidence:

$$
\begin{aligned}
& S_{h}{ }^{\prime}(t)=\Lambda_{h}-\frac{\alpha \beta_{m} I_{v}}{N_{h}} S_{h}-\beta_{p} c\left(I_{p}+k I_{m p}\right) S_{h}-\mu_{h} S_{h}+\pi I_{m}+\tau I_{p}+\phi I_{m p} \\
& I_{m}{ }^{\prime}(t)=\frac{\alpha \beta_{m} I_{v}}{N_{h}} S_{h}-\frac{\vartheta \beta_{p} c\left(I_{p}+k I_{m p}\right)}{N_{h}} I_{m}-\left(\sigma_{m}+\pi+\mu_{h}\right) I_{m} \\
& I_{p}{ }^{\prime}(t)=\frac{\beta_{p} c\left(I_{p}+k I_{m p}\right)}{N_{h}} S_{h}-\frac{\varepsilon \alpha \beta_{m} I_{v}}{N_{h}} I_{p}-\left(\sigma_{p}+\tau+\mu_{h}\right) I_{p} \\
& I_{m p}{ }^{\prime}(t)=\frac{\varepsilon \alpha \beta_{m} I_{v}}{N_{h}} I_{p}+\frac{\vartheta \beta_{p} c\left(I_{p}+k I_{m p}\right)}{N_{h}} I_{m}-\left(\sigma_{m}+\sigma_{p}+\sigma_{m p}+\phi+\mu_{h}\right) I_{m p} \\
& S_{v}{ }^{\prime}(t)=\Lambda_{v} N_{v}-\frac{\alpha \beta_{v} c\left(I_{m}+\delta I_{m p}\right)}{N_{h}} S_{v}-\mu_{v} S_{v} \\
& I_{v}{ }^{\prime}(t)=\frac{\alpha \beta_{v} c\left(I_{m}+\delta I_{m p}\right)}{N_{h}} S_{v}-\mu_{v} I_{v}
\end{aligned}
$$

Cai et al [11] replaced the standard incidence with the mass action incidence in their study of malaria model with partial immunity to reinfection. Thus, following the modification of the work of Lawi et al [24], Oluyo and Adeniyi [29] studied the following model (2.2) on the coinfection of malaria-pneumonia using the mass action incidence. 


$$
\begin{aligned}
& S_{h}{ }^{\prime}(t)=\Lambda_{h}-\alpha \beta_{m} I_{v} S_{h}-\beta_{p} c\left(I_{p}+k I_{m p}\right) S_{h}-\mu_{h} S_{h}+\pi I_{m}+\tau I_{p}+\phi I_{m p} \\
& I_{m}{ }^{\prime}(t)=\alpha \beta_{m} I_{v} S_{h}-\vartheta \beta_{p} c\left(I_{p}+k I_{m p}\right) I_{m}-\left(\sigma_{m}+\pi+\mu_{h}\right) I_{m} \\
& I_{p}{ }^{\prime}(t)=\beta_{p} c\left(I_{p}+k I_{m p}\right) S_{h}-\varepsilon \alpha \beta_{m} I_{v} I_{p}-\left(\sigma_{p}+\tau+\mu_{h}\right) I_{p} \\
& I_{m p}{ }^{\prime}(t)=\varepsilon \alpha \beta_{m} I_{v} I_{p}+\vartheta \beta_{p} c\left(I_{p}+k I_{m p}\right) I_{m}-\left(\sigma_{m}+\sigma_{p}+\sigma_{m p}+\phi+\mu_{h}\right) I_{m p} \\
& S_{v}{ }^{\prime}(t)=\Lambda_{v}-\alpha \beta_{v}\left(I_{m}+\delta I_{m p}\right) S_{v}-\mu_{v} S_{v} \\
& I_{v}^{\prime}(t)=\alpha \beta_{v}\left(I_{m}+\delta I_{m p}\right) S_{v}-\mu_{v} I_{v}
\end{aligned}
$$

One intervention recommended to control malaria transmission in the work of [29] was keeping clean environment. For pneumonia, intervention can be in two ways: reducing the number of susceptible individuals through vaccination or reducing the rate of contact by good sanitation. Based on the above submission, the model in (2.2) was further extended to include the exposed classes for malaria, pneumonia and malaria-pneumonia, vaccination for those exposed to pneumonia and the sanitation function for malaria and pneumonia. Thus, the improved model is:

$$
\begin{aligned}
& S_{h}{ }^{\prime}(t)=\Lambda_{h}-\alpha \beta(H) I_{v} S_{h}-\beta_{p} c(H)\left(I_{p}+k I_{m p}\right) S_{h}-\mu_{h} S_{h}+\pi I_{m} \\
& \quad+\pi E_{m}+\tau I_{p}+\tau E_{p}+V_{p} E_{p}+\phi I_{m p}+\phi E_{m p} \\
& E_{m}{ }^{\prime}(t)=\alpha \beta(H) I_{v} S_{h}-\left(k_{m}+\pi+\mu_{h}\right) E_{m} \\
& I_{m}{ }^{\prime}(t)=k_{m} E_{m}-\vartheta \beta_{p} c(H)\left(I_{p}+k I_{m p}\right) I_{m}-\left(\sigma_{m}+\pi+\mu_{h}\right) I_{m} \\
& E_{p}{ }^{\prime}(t)=\beta_{p} c(H)\left(I_{p}+k I_{m p}\right) S_{h}-\left(k_{p}+\tau+V_{p}+\mu_{h}\right) E_{p} \\
& I_{p}{ }^{\prime}(t)=k_{p} E_{p}-\varepsilon \alpha \beta(H) I_{v} I_{p}-\left(\sigma_{p}+\tau+\mu_{h}\right) I_{p} \\
& E_{m p}{ }^{\prime}(t)=\varepsilon \alpha \beta(H) I_{v} I_{p}+\vartheta \beta_{p} c(H)\left(I_{p}+k I_{m p}\right) I_{m}-\left(k_{m p}+\phi+\mu_{h}\right) E_{m p} \\
& I_{m p}{ }^{\prime}(t)=k_{m p} E_{m p}-\left(\sigma_{m}+\sigma_{p}+\sigma_{m p}+\phi+\mu_{h}\right) I_{m p} \\
& S_{v}{ }^{\prime}(t)=\Lambda_{v}-\alpha \beta_{v}\left(I_{m}+\delta I_{m p}\right) S_{v}-\mu_{v} S_{v} \\
& E_{v}{ }^{\prime}(t)=\alpha \beta_{v}\left(I_{m}+\delta I_{m p}\right) S_{v}-\left(k_{v}+\mu_{v}\right) E_{v} \\
& I_{v}{ }^{\prime}(t)=k_{v} E_{v}-\mu_{v} I_{v}
\end{aligned}
$$

The model (2.3) subdivides the total human population of interest into two sub population depending on the malaria and pneumonia status of individuals. The classes consist of Susceptible $(S)$ representing the number of individuals who are at risk of acquiring malaria or pneumonia or both infections, Individuals Exposed to malaria or pneumonia or both infections $(E)$, Infective $(I)$ representing infectious malaria individuals or infectious pneumonia individuals or infectious malaria-pneumonia individuals capable of transmitting infection to susceptible individuals. The constant per capita recruitment rate into susceptible human population is $\Lambda_{h}$ while humans die naturally at a rate $\mu_{h}$, the modification parameter according to the increased susceptibility to infection with pneumonia is denoted $\vartheta$, humans die as a result of malaria infection at rate $\sigma_{m}$ while humans die of pneumonia infection at a rate $\sigma_{p}$. Individual become infected with pneumonia by coming in contact with one infectious individual at rate $\beta_{p}$. Malaria and pneumonia induced mortality occur at rate $\sigma_{m p}$, rate of recovery from malaria, pneumonia and malaria-pneumonia back to the susceptible class are denoted by $\pi, \tau$ and $\phi$ respectively while $\alpha$ denote the number of bites per human per mosquito. The per capita rate of progression of humans from the exposed class $E_{m}$ to the infectious class $I_{m}$ is $k_{m}$ with $\frac{1}{k_{m}}$ taking as the average duration of the latent period, $k_{p}$ represent the per capita rate of progression 
of humans from the exposed class $E_{p}$ to the infectious class $I_{p}$ with $\frac{1}{k_{p}}$ denoting the average duration of the latent period while $k_{m p}$ is the per capita rate of progression of humans from the exposed class $E_{m p}$ to the infectious class $I_{m p} \cdot \frac{1}{k_{m p}}$ is the average duration of the latent period. Exposed individuals to pneumonia are vaccinated at constant rate $V_{p}$. The mosquitoes (vector) population are sub-divided into susceptible mosquitoes $\left(S_{v}\right)$, mosquitoes exposed to malaria $\left(E_{v}\right)$ and infected mosquitoes $\left(I_{v}\right)$ respectively. The per capita recruitment rate of mosquitos into susceptible vector population is $\Lambda_{v}$, mosquito become infected with malaria after taking a blood from any infected human with malaria at a rate $\beta_{v}$, exposed mosquitoes $\left(E_{v}\right)$ progress to the infectious class $\left(I_{v}\right)$ at a constant rate $k_{v}$ with $\frac{1}{k_{v}}$ being the average duration of the latent period, the mosquito natural death rate is $\mu_{v} . \varepsilon$, denote the expected decrease in contact due to ill health as a result of pneumonia disease such that $0<\varepsilon<1$. The modification parameters accounting for the relative infectiousness of the co-infected individual as compared to their counterparts are $\delta$ and $k$ respectively. $\beta(H)$ is the transmission rate of malaria in humans expressed as function (linearly decreasing function) of the effectiveness of sanitation $H$ and is defined by

$\beta(H)=\beta_{\max }-\gamma H$

$\gamma$ represent the expected reduction / increase in transmission rate of malaria as sanitation level $H$ increases / decreases. $H$ is the sanitation level of the community and is defined to be $H \in[0,1]$, so that if $H=0$, then there is maximum transmission of malaria in the community and if $H=1$ means there is access to maximum sanitation facilities in the community, hence, minimum transmission rate of malaria is achieved. $C(H)$ is the contracting rate of pneumonia expressed as function (linearly decreasing function) of the effectiveness of sanitation $H$ and is defined by

$$
C(H)=C_{\max }-\gamma H
$$

when $H=0$, then there is maximum transmission of pneumonia in the community and when $H=$ 1 means there is minimum contacting rate of pneumonia in the community.

The assumptions for the model in system of equations (2.3) are as follows:

i. This model assumes a homogeneous mixing of individuals in the population where all individuals have equal likelihood of contracting the infection if they come into effective contact with infectious individuals or infectious mosquitoes and that transmission of the infection occurs with a mass action incidence rate.

ii. Infected individuals are assumed to recover with no permanent immunity and return to the susceptible class.

iii. Human population is assumed not to be constant since birth, immigration, emigration and death occur in the population.

iv. The probability of survival till the infectious state for individual exposed to malaria as well as those exposed to pneumonia is less or equal to unity.

v. That as a result of the assumption (iv) above, the exposed class of individuals in the population are therefore included.

\subsection{Malaria - only sub model}

Before the full model of system (2.3) is analysed, it is important to gain insight into the dynamics of the malaria - only sub model obtained by setting $E_{p}(t)=E_{m p}(t)=I_{p}(t)=$ $I_{m p}(t)=0$ in (2.3) given by 


$$
\begin{aligned}
& S_{h}{ }^{\prime}(t)=\Lambda_{h}-\alpha \beta(H) I_{v} S_{h}-\mu_{h} S_{h}+\pi E_{m}+\pi I_{m} \\
& E_{m}{ }^{\prime}(t)=\alpha \beta(H) I_{v} S_{h}-\left(k_{m}+\pi+\mu_{h}\right) E_{m} \\
& I_{m}{ }^{\prime}(t)=k_{m} E_{m}-\left(\sigma_{m}+\pi+\mu_{h}\right) I_{m} \\
& S_{v}{ }^{\prime}(t)=\Lambda_{v}-\alpha \beta_{v} I_{m} S_{v}-\mu_{v} S_{v} \\
& E_{v}{ }^{\prime}(t)=\alpha \beta_{v} I_{m} S_{v}-\left(k_{v}+\mu_{v}\right) E_{v} \\
& I_{v}{ }^{\prime}(t)=k_{v} E_{v}-\mu_{v} I_{v}
\end{aligned}
$$

Let the total human population and vector population at any time $t$ be denote by $N_{h}(t)$ and $N_{v}(t)$ respectively, then the total human and vector population for the malaria - only model of (3.1) are given by

$N_{h}(t)=S_{h}(t)+E_{m}(t)+I_{m}(t)$

and

$N_{v}(t)=S_{v}(t)+E_{v}(t)+I_{v}(t)$

respectively. It is easy to show that both human and vector population will reach a constant value over time. i.e. $N_{h}(t)=\frac{\Lambda_{h}}{\mu_{h}}$ and $N_{v}(t)=\frac{\Lambda_{v}}{\mu_{v}}$.

For system (3.1), it is straightforward to verify that the region $\Gamma_{h v}=\left\{\left(S_{h}, E_{m}, I_{m}, S_{v}, E_{v}, I_{v}\right) \in\right.$ $\left.\mathcal{R}_{+}{ }^{6}: S_{h}+E_{m}+I_{m} \leq \frac{\Lambda_{h}}{\mu_{h}}, S_{v}+E_{v}+I_{v} \leq \frac{\Lambda_{v}}{\mu_{v}}\right\}$ is positively invariant and attracting. Thus the dynamics of malaria - only model will be analysed in $\Gamma_{h v}$.

The malaria - only model of (3.1) has a disease-free equilibrium point given by

$M_{0}=\left(S_{h 0}{ }^{*}, E_{m 0}{ }^{*}, I_{m 0}{ }^{*}, S_{v 0}{ }^{*}, E_{v 0}{ }^{*}, I_{v 0}{ }^{*}\right)=\left(\frac{\Lambda_{h}}{\mu_{h}}, 0,0, \frac{\Lambda_{v}}{\mu_{v}}, 0,0\right)$

and endemic equilibriums $M_{1}{ }^{*}=\left(S_{h 1}{ }^{*}, E_{m 1}{ }^{*}, I_{m 1}{ }^{*}, S_{v 1}{ }^{*}, E_{v 1}{ }^{*}, I_{v 1}{ }^{*}\right)$ and $M_{2}{ }^{*}=\left(S_{h 2}{ }^{*}, E_{m 2}{ }^{*}, I_{m 2}{ }^{*}, S_{v 2}{ }^{*}, E_{v 2}{ }^{*}, I_{v 2}{ }^{*}\right)$ given by the positive roots of

$A I_{m}^{* 2}+B I_{m}^{*}+C=0$

with

$$
\begin{aligned}
& A=\alpha^{3} \beta(H) \beta_{v}{ }^{2} k_{m} k_{v} \Lambda_{v} \pi\left(\sigma_{m}+\pi+\mu_{h}\right)+\alpha^{3} \beta_{v}{ }^{2} k_{m} \pi-\left(k_{m}+\pi+\mu_{h}\right)\left(\sigma_{m}+\pi+\right. \\
& \left.\mu_{h}\right) \alpha^{3} \beta(H) \beta_{v}{ }^{2} k_{v} \Lambda_{v}-\left(k_{m}+\pi+\mu_{h}\right)\left(\sigma_{m}+\pi+\mu_{h}\right) \alpha^{2} \beta_{v}{ }^{2} \mu_{h} \mu_{v}\left(k_{v}+\mu_{v}\right)
\end{aligned}
$$

$B=\alpha^{3} \beta(H) \beta_{v}{ }^{2} k_{m} k_{v} \Lambda_{v} \Lambda_{h}+\alpha^{2} \beta(H) \beta_{v} k_{m} k_{v} \Lambda_{v} \mu_{v} \pi\left(\sigma_{m}+\pi+\mu_{h}\right)+$

$\alpha^{2} \beta(H) \beta_{v} k_{m}{ }^{2} k_{v} \Lambda_{v} \mu_{v} \pi-2\left(k_{m}+\pi+\mu_{h}\right)\left(\sigma_{m}+\pi+\mu_{h}\right) \alpha \beta_{v} \mu_{v}{ }^{2} \mu_{h}\left(k_{v}+\mu_{v}\right)-$

$\alpha^{2} \beta(H) \beta_{v} \mu_{v} k_{v} \Lambda_{v}\left(k_{m}+\pi+\mu_{h}\right)\left(\sigma_{m}+\pi+\mu_{h}\right)$

$C=k_{m} k_{v} \alpha^{2} \beta(H) \beta_{v} \Lambda_{v} \Lambda_{h} \mu_{v}-\mu_{h} \mu_{v}{ }^{3}\left(k_{m}+\pi+\mu_{h}\right)\left(\sigma_{m}+\pi+\mu_{h}\right)\left(k_{v}+\mu_{v}\right)$

The local stability of the equilibrium $M_{0}$ is governed by the basic reproduction number $R_{m}$. The basic reproduction number for the malaria model of (3.1) will be investigated using the next generation approach ( [15], [35]). Using the notations in [35] on system (3.1), the matrices $F$ and $V$, representing the new infections terms and transferred terms respectively are given by

$$
F=\left(\begin{array}{cccc}
0 & 0 & 0 & \left(\alpha \beta_{v} \Lambda_{v} / \mu_{v}\right) \\
0 & 0 & 0 & 0 \\
0 & \left(\alpha \beta_{v} \Lambda_{v} / \mu_{v}\right) & 0 & 0 \\
0 & 0 & 0 & 0
\end{array}\right)
$$




$$
V=\left(\begin{array}{cccc}
\left(k_{m}+\pi+\mu_{h}\right) & 0 & 0 & 0 \\
-k_{m} & \left(\sigma_{m}+\pi+\mu_{h}\right) & 0 & 0 \\
0 & 0 & \left(k_{v}+\mu_{v}\right) & 0 \\
0 & 0 & -k_{v} & \mu_{v}
\end{array}\right)
$$

Thus, the basic reproduction number for system (3.1), denoted by $R_{m}$ is given by the spectral radius $\rho$ of $F V^{-1}$ (the dominant eigenvalue in magnitude)

$$
R_{m}=\rho\left(F V^{-1}\right)=\sqrt{\frac{\alpha^{2} \beta(H) \beta_{v} k_{m} k_{v} \Lambda_{h} \Lambda_{v}}{\mu_{h} \mu_{v}^{2}\left(k_{v}+\mu_{v}\right)\left(\sigma_{m}+\pi+\mu_{h}\right)\left(k_{m}+\pi+\mu_{h}\right)}}
$$

The reproduction number $R_{m}$ is the average number of secondary malaria infectious cases produced by a single infected mosquito during its infectious period when introduced in a population of mostly malaria susceptible individuals.

Theorem 3.1: The malaria disease - free equilibrium $M_{0}$ of system (3.1) is locally asymptotically stable if $R_{m}<1$ and unstable if $R_{m}>1$.

Proof: The Jacobian matrix of system (3.1) evaluated at $M_{0}$ is $J\left(M_{0}\right)=$

$\left(\begin{array}{cccccc}-\mu_{h} & \pi & \pi & 0 & 0 & \left(-\alpha \beta(H) \Lambda_{h} / \mu_{h}\right) \\ 0 & -\left(k_{m}+\pi+\mu_{h}\right) & 0 & 0 & 0 & \left(\alpha \beta(H) \Lambda_{h} / \mu_{h}\right) \\ 0 & k_{m} & -\left(\sigma_{m}+\pi+\mu_{h}\right) & 0 & 0 & 0 \\ 0 & 0 & \left(-\alpha \beta_{v} \Lambda_{v} / \mu_{v}\right) & -\mu_{v} & 0 & 0 \\ 0 & 0 & \left(\alpha \beta_{v} \Lambda_{v} / \mu_{v}\right) & 0 & -\left(k_{v}+\mu_{v}\right) & 0 \\ 0 & 0 & 0 & 0 & k_{v} & -\mu_{v}\end{array}\right)$

The characteristic equation of equation (3.7) is

$\left(-\mu_{h}-\lambda\right)\left(-\mu_{v}-\lambda\right) g(\lambda)=0$

where

$g(\lambda)=\left(\lambda^{4}+\left(k_{m}+2 \pi+2 \mu_{h}+2 \mu_{v}+\sigma_{m}+k_{v}\right) \lambda^{3}+\left(k_{m}+\pi+\mu_{h}\right)\left(\sigma_{m}+\pi+\mu_{h}\right)+\right.$ $\left.\left(k_{m}+2 \pi+2 \mu_{h}+\sigma_{m}\right)\left(k_{v}+\mu_{v}\right)+\left(k_{m}+2 \pi+2 \mu_{h}+2 \mu_{v}+\sigma_{m}+k_{v}\right) \mu_{v}\right) \lambda^{2}+\left(\mu_{v}\left(\left(k_{m}+\pi+\right.\right.\right.$ $\left.\left.\mu_{h}\right)\left(\sigma_{m}+\pi+\mu_{h}\right)+\left(k_{m}+2 \pi+2 \mu_{h}+\sigma_{m}\right)\left(k_{v}+\mu_{v}\right)\right)+\left(k_{m}+\pi+\mu_{h}\right)\left(\sigma_{m}+\pi+\mu_{h}\right)\left(k_{v}+\right.$ $\left.\left.\mu_{v}\right)\right) \lambda+\mu_{v}\left(k_{v}+\mu_{v}\right)\left(\sigma_{m}+\pi+\mu_{h}\right)\left(k_{m}+\pi+\mu_{h}\right)-\frac{\alpha^{2} \beta(H) \beta_{v} k_{m} k_{v} \Lambda_{h} \Lambda_{v}}{\mu_{h} \mu_{v}}$

Clearly, $\lambda_{1}=-\mu_{h}, \lambda_{2}=-\mu_{v}$ while $\lambda_{3}, \lambda_{4}, \lambda_{5}$ and $\lambda_{6}$ are obtained from $g(\lambda)=0$ i.e $\lambda^{4}+\left(k_{m}+2 \pi+2 \mu_{h}+2 \mu_{v}+\sigma_{m}+k_{v}\right) \lambda^{3}+\left(\left(k_{m}+\pi+\mu_{h}\right)\left(\sigma_{m}+\pi+\mu_{h}\right)+\left(k_{m}+2 \pi+\right.\right.$ $\left.\left.2 \mu_{h}+\sigma_{m}\right)\left(k_{v}+\mu_{v}\right)+\left(k_{m}+2 \pi+2 \mu_{h}+2 \mu_{v}+\sigma_{m}+k_{v}\right) \mu_{v}\right) \lambda^{2}+\left(\mu_{v}\left(\left(k_{m}+\pi+\mu_{h}\right)\left(\sigma_{m}+\right.\right.\right.$ $\left.\left.\left.\pi+\mu_{h}\right)+\left(k_{m}+2 \pi+2 \mu_{h}+\sigma_{m}\right)\left(k_{v}+\mu_{v}\right)\right)+\left(k_{m}+\pi+\mu_{h}\right)\left(\sigma_{m}+\pi+\mu_{h}\right)\left(k_{v}+\mu_{v}\right)\right) \lambda+$ $\mu_{v}\left(k_{v}+\mu_{v}\right)\left(\sigma_{m}+\pi+\mu_{h}\right)\left(k_{m}+\pi+\mu_{h}\right)-\frac{\alpha^{2} \beta(H) \beta_{v} k_{m} k_{v} \Lambda_{h} \Lambda_{v}}{\mu_{h} \mu_{v}}=0$

Equation (3.9) will have four negative real roots (by Descartes rule of positive solutions) if $\frac{\alpha^{2} \beta(H) \beta_{v} k_{m} k_{v} \Lambda_{h} \Lambda_{v}}{\mu_{h} \mu_{v}}<\mu_{v}\left(k_{v}+\mu_{v}\right)\left(\sigma_{m}+\pi+\mu_{h}\right)\left(k_{m}+\pi+\mu_{h}\right)$

$\Rightarrow \frac{\alpha^{2} \beta(H) \beta_{v} k_{m} k_{v} \Lambda_{h} \Lambda_{v}}{\mu_{h} \mu_{v}^{2}\left(k_{v}+\mu_{v}\right)\left(\sigma_{m}+\pi+\mu_{h}\right)\left(k_{m}+\pi+\mu_{h}\right)}<1$

$R_{m}^{2}<1$

$\therefore R_{m}<1$ 
Hence, the malaria disease - free equilibrium $M_{0}$ of system (3.1) is locally asymptotically stable if $R_{m}<1$. The result follows immediately that $M_{0}$ is unstable if $R_{m}>1$.

Theorem 3.2: The malaria disease - free equilibrium $M_{0}$ of system (3.1) is globally asymptotically stable if $R_{m}<1$ and unstable if $R_{m}>1$.

Proof:The Comparison method as implemented in Lashmkantham, et al [23] and Mushayabasa et al [27] is used here. The rate of change of the exposed and infected components of system (3.1) can be written as

$\left(\begin{array}{l}\frac{d E_{m}}{d t} \\ \frac{d I_{m}}{d t} \\ \frac{d E_{v}}{d t} \\ \frac{d I_{v}}{d t}\end{array}\right)=(F-V)\left(\begin{array}{c}E_{m} \\ I_{m} \\ E_{v} \\ I_{v}\end{array}\right)-\left(1-\frac{S_{h}}{N_{h}}\right)\left(1-\frac{S_{v}}{N_{v}}\right) F\left(\begin{array}{c}E_{m} \\ I_{m} \\ E_{v} \\ I_{v}\end{array}\right)$

where $F$ and $V$ are as defined above. Since at the disease free $E_{m}=I_{m}=E_{v}=I_{v}=0 \rightarrow(0,0,0,0)$ and $S_{h} \leq N_{h}, S_{v} \leq N_{v}$ as $t \rightarrow \infty$ in $\Gamma_{h v}$. Thus,

$\left(\begin{array}{l}\frac{d E_{m}}{d t} \\ \frac{d I_{m}}{d t} \\ \frac{d E_{v}}{d t} \\ \frac{d I_{v}}{d t}\end{array}\right) \leq(F-V)\left(\begin{array}{c}E_{m} \\ I_{m} \\ E_{v} \\ I_{v}\end{array}\right)$

Then all eigenvalues of the matrix $(F-V)$ have negative real parts i.e

$\lambda^{4}+\left(k_{m}+2 \pi+2 \mu_{h}+2 \mu_{v}+\sigma_{m}+k_{v}\right) \lambda^{3}+\left(\left(k_{m}+\pi+\mu_{h}\right)\left(\sigma_{m}+\pi+\mu_{h}\right)+\left(k_{m}+2 \pi+\right.\right.$ $\left.\left.2 \mu_{h}+\sigma_{m}\right)\left(k_{v}+\mu_{v}\right)+\left(k_{m}+2 \pi+2 \mu_{h}+2 \mu_{v}+\sigma_{m}+k_{v}\right) \mu_{v}\right) \lambda^{2}+\left(\mu_{v}\left(\left(k_{m}+\pi+\mu_{h}\right)\left(\sigma_{m}+\right.\right.\right.$ $\left.\left.\left.\pi+\mu_{h}\right)+\left(k_{m}+2 \pi+2 \mu_{h}+\sigma_{m}\right)\left(k_{v}+\mu_{v}\right)\right)+\left(k_{m}+\pi+\mu_{h}\right)\left(\sigma_{m}+\pi+\mu_{h}\right)\left(k_{v}+\mu_{v}\right)\right) \lambda+$ $\mu_{v}\left(k_{v}+\mu_{v}\right)\left(\sigma_{m}+\pi+\mu_{h}\right)\left(k_{m}+\pi+\mu_{h}\right)-\frac{\alpha^{2} \beta(H) \beta_{v} k_{m} k_{v} \Lambda_{h} \Lambda_{v}}{\mu_{h} \mu_{v}}=0$

Equation (3.12) have four negative roots by Descartes rule of signs if $R_{m}<1$. It follows that the linearized differential inequality (3.11) is stable whenever $R_{m}<1$. Consequently, $\left(E_{m}, I_{m}, E_{v}, I_{v}\right) \rightarrow(0,0,0,0)$ as $t \rightarrow \infty$. Evaluating system (3.1) at $E_{m}=I_{m}=E_{v}=I_{v}=0$ gives, $S_{h} \rightarrow 1$ and $S_{v} \rightarrow 1$ for $R_{m}<1$. Hence, the malaria diseases free equilibrium $M_{0}$ of system (3.1) is globally asymptotically stable if $R_{m}<1$. The result also follow immediately that the malaria disease - free equilibrium $M_{0}$ of system (3.1) is unstable if $R_{m}>1$.

\subsection{Local Asymptotic Stability of Malaria Endemic Equilibrium}

The Centre Manifold theorem is now applied to study the local stability of the malaria endemic equilibrium.

Let $\beta(H)=\beta(H)^{*}$ be a bifurcation parameter and if the case $R_{m}=1$ is considered, then

$\beta(H)=\beta(H)^{*}=\frac{\mu_{h} \mu_{v}^{2}\left(k_{v}+\mu_{v}\right)\left(k_{m}+\pi+\mu_{h}\right)\left(\sigma_{m}+\pi+\mu_{h}\right)}{\alpha^{2} \beta_{v} k_{m} k_{v} \Lambda_{h} \Lambda_{v}}$

Firstly, the Jacobian matrix of system (3.1) at point $\left(M_{0}, \beta(H)^{*}\right)$ is 


$$
\begin{aligned}
& J\left(M_{0}, \beta(H)^{*}\right) \\
& =\left(\begin{array}{cccccc}
-\mu_{h} & \pi & \pi & 0 & 0 & \left(-\alpha \beta(H)^{*} \Lambda_{h} / \mu_{h}\right) \\
0 & -\left(k_{m}+\pi+\mu_{h}\right) & 0 & 0 & 0 & \left(\alpha \beta(H)^{*} \Lambda_{h} / \mu_{h}\right) \\
0 & k_{m} & -\left(\sigma_{m}+\pi+\mu_{h}\right) & 0 & 0 & 0 \\
0 & 0 & \left(-\alpha \beta_{v} \Lambda_{v} / \mu_{v}\right) & -\mu_{v} & 0 & 0 \\
0 & 0 & \left(\alpha \beta_{v} \Lambda_{v} / \mu_{v}\right) & 0 & -\left(k_{v}+\mu_{v}\right) & 0 \\
0 & 0 & 0 & 0 & k_{v} & -\mu_{v}
\end{array}\right)
\end{aligned}
$$

The characteristic equation of (3.14) has a simple zero eigenvalue i.e.

$\lambda_{1}=-\mu_{h}, \lambda_{2}=-\mu_{v}, \lambda_{3}=0$, while the remaining equation is written as

$\lambda^{3}+\left(k_{m}+2 \pi+2 \mu_{h}+2 \mu_{v}+\sigma_{m}+k_{v}\right) \lambda^{2}+\left(\left(k_{m}+\pi+\mu_{h}\right)\left(\sigma_{m}+\pi+\mu_{h}\right)+\left(k_{m}+2 \pi+\right.\right.$

$\left.\left.2 \mu_{h}+\sigma_{m}\right)\left(k_{v}+\mu_{v}\right)+\left(k_{m}+2 \pi+2 \mu_{h}+2 \mu_{v}+\sigma_{m}+k_{v}\right) \mu_{v}\right) \lambda+\left(\mu_{v}\left(\left(k_{m}+\pi+\mu_{h}\right)\left(\sigma_{m}+\right.\right.\right.$

$\left.\left.\left.\pi+\mu_{h}\right)+\left(k_{m}+2 \pi+2 \mu_{h}+\sigma_{m}\right)\left(k_{v}+\mu_{v}\right)\right)+\left(k_{m}+\pi+\mu_{h}\right)\left(\sigma_{m}+\pi+\mu_{h}\right)\left(k_{v}+\mu_{v}\right)\right)=0$

Equation (3.15) has three negative eigenvalues as its roots (by Descartes rule of signs). Thus, $\lambda_{3}=$ 0 is a simple zero eigenvalue and the other eigenvalues are real and negative, then the assumptions of theorem A.1 (Centre Manifold theorem) in the appendix is then verified. Furthermore, the right and left eigenvectors associated with the zero eigenvalue $\lambda_{3}=0$ are

$w$

$=\left[\begin{array}{l}\frac{\left(\pi \mu_{h} \mu_{v}{ }^{2}\left(k_{v}+\mu_{v}\right)\left(\sigma_{m}+\pi+\mu_{h}\right)+\pi \mu_{h} \mu_{v}{ }^{2} k_{m}\left(k_{v}+\mu_{v}\right)-\alpha^{2} \beta(H)^{*} \beta_{v} k_{m} k_{v} \Lambda_{h} \Lambda_{v}\right) w_{2}}{\mu_{h}\left(k_{v}+\mu_{v}\right)\left(\sigma_{m}+\pi+\mu_{h}\right)}, w_{2}, \\ \frac{k_{m} w_{2}}{\left(\sigma_{m}+\pi+\mu_{h}\right)},-\frac{\alpha \beta_{v} \Lambda_{v} k_{m} w_{2}}{\mu_{v}{ }^{2}\left(\sigma_{m}+\pi+\mu_{h}\right)}, \frac{\alpha \beta_{v} \Lambda_{v} k_{m} w_{2}}{\mu_{v}\left(k_{v}+\mu_{v}\right)\left(\sigma_{m}+\pi+\mu_{h}\right)}, \frac{\alpha \beta_{v} k_{m} k_{v} \Lambda_{v} w_{2}}{\mu_{v}{ }^{2}\left(k_{v}+\mu_{v}\right)\left(\sigma_{m}+\pi+\mu_{h}\right)}\end{array}\right]^{T}$ and

$\bar{v}=\left[\begin{array}{c}0, \bar{v}_{2}, \frac{\left(k_{m}+\pi+\mu_{h}\right) \bar{v}_{2}}{k_{m}}, 0, \frac{\mu_{v}\left(k_{m}+\pi+\mu_{h}\right)\left(\sigma_{m}+\pi+\mu_{h}\right) \bar{v}_{2}}{\Lambda_{v} k_{m} \alpha \beta_{v}} \\ \frac{\left(k_{v}+\mu_{v}\right)\left(k_{m}+\pi+\mu_{h}\right)\left(\sigma_{m}+\pi+\mu_{h}\right) \mu_{v} \bar{v}_{2}}{\Lambda_{v} k_{v} k_{m} \alpha \beta_{v}}\end{array}\right]$

where $w_{2}>0$ and $\bar{v}_{2}>0$ are free right and left eigenvectors.

\section{Computation of the Coefficient $\boldsymbol{a}$ and $\boldsymbol{b}$ for the Malaria Model}

After some rigorous mathematical manipulations using the associated partial non-zero partial derivatives of (3.1) at $M_{0}$, it can be shown that

$\boldsymbol{a}=\frac{2 \bar{v}_{2} \alpha w_{2}^{2} \beta_{v}\left(k_{m}+\pi+\mu_{h}\right)}{\mu_{v}\left(\sigma_{m}+\pi+\mu_{h}\right)}\left[a_{0}-1\right], \quad \boldsymbol{b}=\bar{v}_{2} \frac{\alpha^{2} \beta_{v} k_{m} k_{v} w_{2}}{\mu_{v}\left(k_{v}+\mu_{v}\right)\left(\sigma_{m}+\pi+\mu_{h}\right)}$

where

$a_{0}=\left(\frac{\left(\pi \mu_{h} \mu_{v}{ }^{2}\left(k_{v}+\mu_{v}\right)\left(\sigma_{m}+\pi+\mu_{h}\right)+\pi \mu_{h} \mu_{v}{ }^{2} k_{m}\left(k_{v}+\mu_{v}\right)-\alpha^{2} \beta(H)^{*} \beta_{v} k_{m} k_{v} \Lambda_{h} \Lambda_{v}\right)}{\Lambda_{h} k_{m}\left(k_{v}+\mu_{v}\right)}\right) \mu_{v}$

According to theorem A.1 in the appendix it is the sign of the coefficient $\boldsymbol{a}$ - which depend on $a_{0}$ that decides the local dynamics around the disease free equilibrium for $\beta(H)=\beta(H)^{*}$. Thus,

1. If $a_{0}>1$, then $a>0$, thus a backward bifurcation occurs

2. If $a_{0}<1$, then $a<0$, thus a forward bifurcation occurs

The foregoing discussion is summarized in the following results from theorem A.1 items (a) and (d):

Lemma 3.1: The malaria only model of (3.1) has a positive endemic equilibrium which is unstable 
if $R_{m}<1$ otherwise stable.

Lemma 3.2: The malaria only model of (3.1) has a unique positive endemic equilibrium which is locally asymptotically stable (LAS) if $R_{m}>1$ otherwise unstable.

\subsection{Sensitivity Analysis of $\left(R_{m}\right)$}

The sensitivity index of the reproduction number is used to assess the impact on the relevant parameters to disease transmission. The sensitivity of the reproduction number, $R_{m}$ is analysed to determine the parameter that are most sensitive to $R_{m}$. Following the approach in [6], the forward sensitivity index with respect to biting rates of the mosquitoes, $\alpha$ and treatment rate, $\pi$ used in the malaria only model is presented below using the following formula

$r_{q}^{R_{m}}=\frac{\partial R_{m}}{\partial q} \times \frac{q}{R_{m}}$

$\frac{\partial R_{m}}{\partial \alpha} \times \frac{q \alpha}{R_{m}}=1$

$\frac{\partial R_{m}}{\partial \pi} \times \frac{\pi}{R_{m}}=-\frac{\pi\left(k_{m}+\sigma_{m}+2 \pi+2 \mu_{h}\right)}{2\left(\sigma_{m}+\pi+\mu_{h}\right)\left(k_{m}+\pi+\mu_{h}\right)}$

From the calculation in (3.17), it was observed that $R_{m}$ is most sensitive to changes in the biting rates of the mosquitoes, $\alpha$. An increase in number of mosquito bites $\alpha$ will bring about an increase of the same proportion in $R_{m}$ and a decrease in $\alpha$ will result in a decrease in $R_{m}$ with about an equivalent magnitude. Equation (3.18) imply that an increase in treatment of malaria infected individuals have a positive impact in controlling malaria in the population.

\subsection{Pneumonia only Disease Transmission Model}

Here, pneumonia disease transmission model only are investigated and analysed.

Let $E_{m}(t)=I_{m}(t)=E_{v}(t)=I_{v}(t)=E_{m p}(t)=I_{m p}(t)=0$ in model system (2.3), then we have the following system of equations is obtained

$\left.\begin{array}{l}S_{h}{ }^{\prime}(t)=\Lambda_{h}-\beta_{p} c(H) I_{p} S_{h}-\mu_{h} S_{h}+\tau E_{p}+\tau I_{p}+V_{p} E_{p} \\ E_{p}{ }^{\prime}(t)=\beta_{p} c(H) I_{p} S_{h}-\left(k_{p}+\tau+V_{p}+\mu_{h}\right) E_{p} \\ I_{p}{ }^{\prime}(t)=k_{p} E_{p}-\left(\sigma_{p}+\tau+\mu_{h}\right) I_{p} \\ S_{v}{ }^{\prime}(t)=\Lambda_{v}-\mu_{v} S_{v}\end{array}\right\}$

Note that the fourth equation in system (3.19) is independent of the other three equations then equation (3.19) can be re-written as

$\left.\begin{array}{l}S_{h}{ }^{\prime}(t)=\Lambda_{h}-\beta_{p} c(H) I_{p} S_{h}-\mu_{h} S_{h}+\tau E_{p}+\tau I_{p}+V_{p} E_{p} \\ E_{p}{ }^{\prime}(t)=\beta_{p} c(H) I_{p} S_{h}-\left(k_{p}+\tau+V_{p}+\mu_{h}\right) E_{p} \\ I_{p}{ }^{\prime}(t)=k_{p} E_{p}-\left(\sigma_{p}+\tau+\mu_{h}\right) I_{p}\end{array}\right\}$

with $N_{h}(t)=S_{h}(t)+E_{p}(t)+I_{p}(t)$. For system (3.20) it can be shown that the region $\Gamma=\Gamma_{h} \subset$ $\mathcal{R}_{+}{ }^{3}$ with $\Gamma_{h}=\left\{\left(S_{h}, E_{p}, I_{p}\right) \in \mathcal{R}_{+}{ }^{3}: S_{h}+E_{p}+I_{p} \leq \frac{\Lambda_{h}}{\mu_{h}}\right\}$

is positively invariant. Thus, in this region the model system (3.20) can be considered to be epidemiologically well-posed. The pneumonia free equilibrium is obtained to be

$P_{0}=\left(S_{h 0}{ }^{*}, E_{p 0}{ }^{*}, I_{p 0}{ }^{*}\right)=\left(\frac{\Lambda_{h}}{\mu_{h}}, 0,0\right)$

Following the approach in [15] and [35] as in section 3.0 on the analysis of malaria model. It can be shown that the reproduction number for system (3.20) denoted by $R_{p}$ is given by 
$R_{p}=\frac{k_{p} \beta_{p} c(H) \Lambda_{h}}{\mu_{h}\left(\sigma_{p}+\tau+\mu_{h}\right)\left(k_{p}+\tau+V_{p}+\mu_{h}\right)}$

The basic reproduction number $R_{p}$ is the average number of new infections caused by a single pneumonia infected individual (but not infected with malaria) during his or her infectious period in a population of pneumonia susceptible $\mathrm{s}$ who have no malaria.

\subsubsection{Sensitivity Analysis of the Reproduction Number, $\boldsymbol{R}_{p}$}

Here, the impact of treatment on pneumonia is investigated using the sensitivity of the reproduction number for pneumonia $R_{p}$. It follows from (3.22), that the sensitivity of $R_{p}$ with respect to $\tau$ can be obtained as

$$
\frac{\partial R_{p}}{\partial q} \times \frac{q}{R_{p}}=-\frac{\tau\left(k_{p}+\sigma_{p}+2 \tau+2 \mu_{h}\right)}{\left(k_{p}+\tau+\mu_{h}\right)\left(\sigma_{p}+\tau+\mu_{h}\right)}
$$

Hence, the treatment of pneumonia patients will have a positive impact in reducing pneumonia burden. Thus the foregoing discussion is summarized in the following result:

Theorem 3.3: The pneumonia disease - free equilibrium $P_{0}$ of system (3.20) is locally asymptotically stable if $R_{p}<1$ and unstable if $R_{p}>1$.

Theorem 3.3 implies that pneumonia can be eliminated from the population (when $R_{p}<1$ ) if the initial size of the sub-population of the model system (3.20) are in the basin of attraction of the disease free equilibrium $P_{0}$. In order to ensure that pneumonia elimination does not depend on the initial size of the sub-populations, it is important to establish that the DFE is globally asymptotically stable.

\subsubsection{Global Stability of Pneumonia - Free Equilibrium}

The rate of change of the exposed and the infected components of system (3.25) can be written as $\left(\begin{array}{l}\frac{d E_{p}}{d t} \\ \frac{d I_{p}}{d t}\end{array}\right)=(F-V)\left(\begin{array}{c}E_{p} \\ \mathrm{I}_{p}\end{array}\right)-\left(1-\frac{S_{h}}{N_{h}}\right) F\left(\begin{array}{c}E_{p} \\ \mathrm{I}_{p}\end{array}\right)$

where $F=\left(\begin{array}{cc}0 & \frac{\beta_{p} c(H) \Lambda_{h}}{\mu_{h}} \\ 0 & 0\end{array}\right) \quad, \quad V=\left(\begin{array}{cc}\left(k_{p}+\tau+V_{p}+\mu_{h}\right) & 0 \\ -k_{p} & \left(\sigma_{p}+\tau+\mu_{h}\right)\end{array}\right)$

Since at the disease free $E_{p}=I_{p}=0 \rightarrow(0,0)$ and $S_{h} \leq N_{h}$ as $t \rightarrow \infty$ in $\Gamma_{h}$. Thus, $\left(\begin{array}{l}\frac{d E_{p}}{d t} \\ \frac{d I_{p}}{d t}\end{array}\right) \leq(F-V)\left(\begin{array}{c}E_{p} \\ \mathrm{I}_{p}\end{array}\right)$

Then all eigenvalues of the matrix $(F-V)$ have negative real parts i.e $\lambda^{2}+\left(k_{p}+2 \tau+V_{p}+2 \mu_{h}+\sigma_{p}\right) \lambda+\left(k_{p}+\tau+V_{p}+\mu_{h}\right)\left(\sigma_{p}+\tau+\mu_{h}\right)-k_{p} \beta_{p} c(H)=0$

Equation (3.26) has its entire roots negative and real if $R_{p}<1$

It follows that the linearized differential inequality (3.25) is stable whenever $R_{p}<1$. Consequently, $\left(E_{p}, I_{p},\right) \rightarrow(0,0)$ as $t \rightarrow \infty$. Evaluating system (3.20) at $E_{p}=I_{p}=0$ gives $S_{h} \rightarrow 1$ for $R_{p}<1$. The result is summarized in the following theorem:

Theorem 3.4: The pneumonia diseases free equilibrium $P_{0}$ of system (3.20) is globally asymptotically stable if $R_{p}<1$ and unstable if $R_{p}>1$. 


\subsubsection{Local Asymptotic Stability of Pneumonia Endemic Equilibrium}

The Centre Manifold Theorem as used in [9] is employed here to establish the local asymptotic stability of the endemic equilibrium. The following change of variables are made in order to apply the Centre Manifold theory: Let $S_{h}=x_{1}, E_{p}=x_{2}, I_{p}=x_{3}$; so that $x_{1}+x_{2}+x_{3}=$ $N_{h}(t)$. Furthermore, let the vector $X=\left(x_{1}, x_{2}, x_{3}\right)^{T}$, then the model in system (3.20) can now be written in the form

$\frac{d X}{d t}=f(x)$, where $f=\left(f_{1}, f_{2}, f_{3}\right)^{T}$. It implies that system (3.20) can be written in term of the new variables as

$$
\left.\begin{array}{l}
\frac{d x_{1}}{d t}=f_{1}=\Lambda_{h}-\beta_{p} c(H) x_{3} x_{1}-\mu_{h} x_{1}+\tau x_{2}+V_{p} x_{2}+\tau x_{3} \\
\frac{d x_{2}}{d t}=f_{2}=\beta_{p} c(H) x_{3} x_{1}-\left(k_{p}+\tau+V_{p}+\mu_{h}\right) x_{2} \\
\frac{d x_{3}}{d t}=f_{3}=k_{p} x_{2}-\left(\sigma_{p}+\tau+\mu_{h}\right) x_{3}
\end{array}\right\}
$$

It can be shown that system (3.26) has a right and left eigenvectors associated with zero eigenvalue at $c(H)=c(H)^{*}$ given by

$w$

$$
=\left(\frac{\left(\mu_{h}\left(\tau+V_{p}\right)\left(\sigma_{p}+\tau+\mu_{h}\right)+k_{p}\left(\mu_{h} \tau-\beta_{p} c(H)^{*} \Lambda_{h}\right)\right) w_{2}}{\mu_{h}^{2}\left(\sigma_{p}+\tau+\mu_{h}\right)}, w_{2}, \frac{k_{p} w_{2}}{\left(\sigma_{p}+\tau+\mu_{h}\right)}\right)^{T}
$$

and

$\bar{v}=\left(\bar{v}_{1}, \bar{v}_{2}, \bar{v}_{3}\right)=\left(0, \bar{v}_{2}, \frac{\left(k_{p}+\tau+V_{p}+\mu_{h}\right) \bar{v}_{2}}{k_{p}}\right)$

where $w_{2}>0$ and $\bar{v}_{2}>0$ are free right eigenvector and left eigenvector.

\section{Computation of the coefficient $a$ and $b$}

For system (3.26), the associated non-zero partial derivatives at $P_{0}$ can be shown to be

$\frac{\partial^{2} f_{2}}{\partial x_{1} \partial x_{3}}=\beta_{p} c(H)^{*}$

From (3.29), it follows that

$a=\frac{2 \bar{v}_{2}\left(k_{p}+\tau+V_{p}+\mu_{h}\right) w_{2}^{2} a_{1}}{\Lambda_{h}\left(\sigma_{p}+\tau+\mu_{h}\right)}, \quad b=\bar{v}_{2} w_{3} \beta_{p}=\frac{\bar{v}_{2} k_{p} \beta_{p} w_{2}}{\left(\sigma_{p}+\tau+\mu_{h}\right)}$

where $a_{1}=\mu_{h}\left(\tau+V_{p}\right)\left(\sigma_{p}+\tau+\mu_{h}\right)+k_{p}\left(\mu_{h} \tau-\Lambda_{h} \beta_{p} c(H)^{*}\right)$

Thus, (i) If $a_{1}>0$, a backward bifurcation occurs (ii) If $a_{1}<0$, a forward bifurcation occurs.

The above analysis is summarized in the following results from theorem A.1 item (d):

Lemma 3.3: The pneumonia only model of (3.20) has a unique positive endemic equilibrium which is locally asymptotically stable (LAS) if $R_{p}>1$ otherwise unstable.

\subsection{Analysis of Malaria-Pneumonia Co-infection Disease Transmission Model}

The full model on the co-infection of Malaria and Pneumonia disease transmission (2.3) is now considered. Firstly, the co-infection dynamics of the full model system (2.3) is reduced to an eight dimensional system by neglecting the susceptible classes for both human and vector populations respectively and also using the fact that

$S_{h}(t)=1-E_{m}(t)-I_{m}(t)-E_{p}(t)-I_{p}(t)-E_{m p}(t)-I_{m p}(t)$ and

$S_{v}(t)=1-E_{v}(t)-I_{v}(t)$. Thus, 


$$
\begin{aligned}
& E_{m}{ }^{\prime}(t)=\alpha \beta(H) I_{v}\left(1-E_{m}-I_{m}-E_{p}-I_{p}-E_{m p}-I_{m p}\right)-\left(k_{m}+\pi+\mu_{h}\right) E_{m} \\
& I_{m}{ }^{\prime}(t)=k_{m} E_{m}-\vartheta \beta_{p} c(H)\left(I_{p}+k I_{m p}\right) I_{m}-\left(\sigma_{m}+\pi+\mu_{h}\right) I_{m} \\
& E_{p}{ }^{\prime}(t)=\beta_{p} c(H)\left(I_{p}+k I_{m p}\right)\left(1-E_{m}-I_{m}-E_{p}-I_{p}-E_{m p}-I_{m p}\right)-\left(k_{p}+\tau+V_{p}+\mu_{h}\right) E_{p} \\
& I_{p}{ }^{\prime}(t)=k_{p} E_{p}-\varepsilon \alpha \beta(H) I_{v} I_{p}-\left(\sigma_{p}+\tau+\mu_{h}\right) I_{p} \\
& E_{m p}{ }^{\prime}(t)=\varepsilon \alpha \beta(H) I_{v} I_{p}+\vartheta \beta_{p} c(H)\left(I_{p}+k I_{m p}\right) I_{m}-\left(k_{m p}+\phi+\mu_{h}\right) E_{m p} \\
& I_{m p}{ }^{\prime}(t)=k_{m p} E_{m p}-\left(\sigma_{m}+\sigma_{p}+\sigma_{m p}+\phi+\mu_{h}\right) I_{m p} \\
& E_{v}{ }^{\prime}(t)=\alpha \beta_{v}\left(I_{m}+\delta I_{m p}\right)\left(1-E_{v}-I_{v}\right)-k_{v} E_{v}-\mu_{v} E_{v} \\
& I_{v}{ }^{\prime}(t)=k_{v} E_{v}-\mu_{v} I_{v}
\end{aligned}
$$

The disease-free equilibrium is given by

$P_{m p f}=\left(E_{m}{ }^{0}, I_{m}{ }^{0}, E_{p}{ }^{0}, I_{p}{ }^{0}, E_{m p}{ }^{0}, I_{m p}{ }^{0}, E_{v}{ }^{0}, I_{v}{ }^{0}\right)=(0,0,0,0,0,0,0,0)$

It can be shown that the basic reproduction number, denoted by $R_{m p}$ for the full malariapneumonia co-infection model (3.31) by using the next generation matrix approach is given by $R_{m p}=\max \left\{R_{m}, R_{p}\right\}$

So that the following result follows from theorem 2 in [35]:

Theorem 3.5: The malaria-pneumonia free equilibrium $\boldsymbol{P}_{\boldsymbol{m p f}}$ of system (3.31) is locally asymptotically stable if $R_{m}<1, R_{p}<1$ and unstable if $R_{m}>1$ and $R_{m}>1$.

\subsubsection{Global Stability of Malaria-Pneumonia Free Equilibrium $\boldsymbol{P}_{\boldsymbol{m p f}}$ Model}

Theorem 3.6: The malaria-pneumonia free equilibrium $\boldsymbol{P}_{\boldsymbol{m p} \boldsymbol{f}}$ of system (3.31) is globally asymptotically stable if $R_{p}<1, R_{m}<1$, otherwise unstable.

\section{Proof:}

The rate of change of the exposed and infected components of system (3.31) can be written as $\left(\begin{array}{c}\frac{d E_{m}}{d t} \\ \frac{d I_{m}}{d t} \\ \frac{d E_{p}}{d t} \\ \frac{d I_{p}}{d t} \\ \frac{d E_{m p}}{d t} \\ \frac{d I_{m p}}{d t} \\ \frac{d E_{v}}{d t} \\ \frac{d I_{v}}{d t}\end{array}\right)=(F-V)\left(\begin{array}{c}E_{m} \\ I_{m} \\ E_{p} \\ I_{p} \\ E_{m p} \\ I_{m p} \\ E_{v} \\ I_{v}\end{array}\right)-\left(1-\frac{S_{h}}{N_{h}}\right)\left(1-\frac{S_{v}}{N_{v}}\right) F\left(\begin{array}{c}E_{m} \\ I_{m} \\ E_{p} \\ I_{p} \\ E_{m p} \\ I_{m p} \\ E_{v} \\ I_{v}\end{array}\right)$

where $F$ and $V$ are given by 
$F=\left(\begin{array}{cccccccc}0 & 0 & 0 & 0 & 0 & 0 & 0 & \alpha \beta(H) \\ 0 & 0 & 0 & 0 & 0 & 0 & 0 & 0 \\ 0 & 0 & 0 & \beta_{p} c(H) & 0 & \beta_{p} k c(H) & 0 & 0 \\ 0 & 0 & 0 & 0 & 0 & 0 & 0 & 0 \\ 0 & 0 & 0 & 0 & 0 & 0 & 0 & 0 \\ 0 & 0 & 0 & 0 & 0 & 0 & 0 & 0 \\ 0 & \alpha \beta_{v} & 0 & 0 & 0 & \alpha \beta_{v} \delta & 0 & 0 \\ 0 & 0 & 0 & 0 & 0 & 0 & 0 & 0\end{array}\right)$

and

$V=\left(\begin{array}{cccccccc}D_{0} & 0 & 0 & 0 & 0 & 0 & 0 & 0 \\ -k_{m} & D_{1} & 0 & 0 & 0 & 0 & 0 & 0 \\ 0 & 0 & D_{2} & 0 & 0 & 0 & 0 & 0 \\ 0 & 0 & -k_{p} & D_{3} & 0 & 0 & 0 & 0 \\ 0 & 0 & 0 & 0 & D_{4} & 0 & 0 & 0 \\ 0 & 0 & 0 & 0 & -k_{m p} & D_{5} & 0 & 0 \\ 0 & 0 & 0 & 0 & 0 & 0 & \left(k_{v}+\mu_{v}\right) & 0 \\ 0 & 0 & 0 & 0 & 0 & 0 & -k_{v} & \mu_{v}\end{array}\right)$

with $D_{0}=\left(k_{m}+\pi+\mu_{h}\right), D_{1}=\left(\sigma_{m}+\pi+\mu_{h}\right), D_{2}=\left(k_{p}+\tau+V_{p}+\mu_{h}\right)$,

$D_{3}=\left(\sigma_{p}+\tau+\mu_{h}\right), D_{4}=\left(k_{m p}+\phi+\mu_{h}\right), D_{5}=\left(\sigma_{m}+\sigma_{p}+\sigma_{m p}+\phi+\mu_{h}\right)$

Since at the disease free $E_{m}=I_{m}=E_{p}=I_{p}=E_{m p}=I_{m p}=E_{v}=I_{v}=0 \rightarrow(0,0,0,0,0,0,0,0)$ with $S_{h} \leq N_{h}$ and $S_{v} \leq N_{v}$ as $t \rightarrow \infty$ in $\Gamma$. Thus,

$\left(\begin{array}{c}\frac{d E_{m}}{d t} \\ \frac{d I_{m}}{d t} \\ \frac{d E_{p}}{d t} \\ \frac{d I_{p}}{d t} \\ \frac{d E_{m p}}{d t} \\ \frac{d I_{m p}}{d t} \\ \frac{d E_{v}}{d t} \\ \frac{d I_{v}}{d t}\end{array}\right) \leq(F-V)\left(\begin{array}{c}E_{m} \\ I_{m} \\ E_{p} \\ I_{p} \\ E_{m p} \\ I_{m p} \\ E_{v} \\ I_{v}\end{array}\right)$

According to [35], all eigenvalues of the matrix $(F-V)$ have negative real parts i.e.

$|(F-V)-\lambda I|=0$

Equation (3.41) simplifies to give

$\left(-D_{4}-\lambda\right)\left(-D_{5}-\lambda\right)\left[\left(-D_{0}-\lambda\right)\left(-D_{1}-\lambda\right)\left(-\mu_{v}-\lambda\right)\left(-\left(k_{v}+\mu_{v}\right)-\lambda\right)-\right.$ $\left.\alpha^{2} \beta_{v} \beta(H) k_{m} k_{v}\right]\left[\left(-D_{2}-\lambda\right)\left(-D_{3}-\lambda\right)-k_{p} \beta_{p} c(H)\right]=0$

Clearly, $\lambda_{1}=-D_{4}, \lambda_{2}=-D_{5}$ and

$\left(-D_{0}-\lambda\right)\left(-D_{1}-\lambda\right)\left(-\mu_{v}-\lambda\right)\left(-\left(k_{v}+\mu_{v}\right)-\lambda\right)-\alpha^{2} \beta_{v} \beta_{m} k_{m} k_{v}=0$ 
$\lambda^{4}+\left(D_{0}+D_{1}+\mu_{v}+\left(k_{v}+\mu_{v}\right)\right) \lambda^{3}+\left(D_{0} D_{1}+D_{0} \mu_{v}+D_{0}\left(k_{v}+\mu_{v}\right)+D_{1} \mu_{v}+D_{1}\left(k_{v}+\mu_{v}\right)+\right.$ $\left.\mu_{v}\left(k_{v}+\mu_{v}\right)\right) \lambda^{2}+\left(D_{0} D_{1} \mu_{v}+D_{0} \mu_{v}\left(k_{v}+\mu_{v}\right)+D_{0} D_{1}\left(k_{v}+\mu_{v}\right)\right) \lambda+D_{0} D_{1} \mu_{v}\left(k_{v}+\mu_{v}\right)-$ $\alpha^{2} \beta_{v} \beta(H) k_{m} k_{v}=0$

Equation (3.37) will have all its eigenvalues to be real and negative (Descartes Rule of signs) if $R_{m}<1$ and $R_{p}<1$. Therefore, all eigenvalues of the linearized differential inequality are negative if $R_{p}<1$ and $R_{m}<1$. Consequently, $\left(E_{m}, I_{m}, E_{p}, I_{p}, E_{m p}, I_{m p}, E_{v}, I_{v}\right) \rightarrow$ $(0,0,0,0,0,0,0,0)$ as $t \rightarrow \infty$. Evaluating system (3.31) at $E_{m}=I_{m}=E_{p}=I_{p}=E_{m p}=I_{m p}=E_{v}=$ $I_{v}=0$ gives $S_{h} \rightarrow 1, S_{v} \rightarrow 1$ for $R_{p}<1$ and $R_{m}<1$. Hence, the malaria-pneumonia disease free equilibrium $\boldsymbol{P}_{\boldsymbol{m p f}}$ is globally asymptotically stable if $R_{p}<1$ and $R_{m}<1$. The result follows that the malaria-pneumonia disease free equilibrium $\boldsymbol{P}_{\boldsymbol{m p f}}$ is unstable if $R_{p}>1$ and $R_{m}>1$.

\subsubsection{Local Asymptotic Stability of Malaria-Pneumonia Endemic Equilibrium $\boldsymbol{P}_{\boldsymbol{m} p \boldsymbol{e}}$}

We establish the stability of the endemic equilibrium of the malaria - pneumonia model (3.31), using the Centre Manifold theory. The following result follows.

Theorem 3.6: The full malaria - pneumonia co-infection model of (3.31) has a unique endemic equilibrium which is locally asymptotically stable if $R_{m p}>1$ and unstable if $R_{m p}<1$ whenever item (d) of theorem A.1 in the appendix is satisfied.

\section{Proof:}

To apply the Centre Manifold theory, the following change of variables is made. Let $E_{m}=x_{1}$, $I_{m}=x_{2}, E_{p}=x_{3}, I_{p}=x_{4}, E_{m p}=x_{5}, I_{m p}=x_{6}, E_{v}=x_{7}, I_{v}=x_{8}$

Furthermore, introducing the vector $X=\left(x_{1}, x_{2}, x_{3}, x_{4}, x_{5}, x_{6}, x_{7}, x_{8}\right)^{T}$, and then the model in system (3.31) can now be written in the form $\frac{d X}{d t}=F(x)$, where $F=\left(f_{1}, f_{2}, f_{3}, f_{4}, f_{5}, f_{6}, f_{7}, f_{8}\right)^{T}$. It implies that system (3.31) can be written in term of the new variable as follow:

$$
\begin{aligned}
& \frac{d x_{1}}{d t}=f_{1}=\alpha \beta(H) x_{8}\left(1-x_{1}-x_{2}-x_{3}-x_{4}-x_{5}-x_{6}\right)-\left(k_{m}+\pi+\mu_{h}\right) x_{1} \\
& \frac{d x_{2}}{d t}=f_{2}=k_{m} x_{1}-\vartheta \beta_{p} c(H)\left(x_{4}+k x_{6}\right) x_{2}-\left(\sigma_{m}+\pi+\mu_{h}\right) x_{2} \\
& \frac{d x_{3}}{d t}=f_{3}=\beta_{p} c(H)\left(x_{4}+k x_{6}\right)\left(1-x_{1}-x_{2}-x_{3}-x_{4}-x_{5}-x_{6}\right)-\left(k_{p}+\tau+V_{p}+\mu_{h}\right) x_{3} \\
& \frac{d x_{4}}{d t}=f_{4}=k_{p} x_{3}-\varepsilon \alpha \beta(H) x_{8} x_{4}-\left(\sigma_{p}+\tau+\mu_{h}\right) x_{4} \\
& \frac{d x_{5}}{d t}=f_{5}=\varepsilon \alpha \beta(H) x_{8} x_{4}+\vartheta \beta_{p} c(H)\left(x_{4}+k x_{6}\right) x_{2}-\left(k_{m p}+\phi+\mu_{h}\right) x_{5} \\
& \frac{d x_{6}}{d t}=f_{6}=k_{m p} x_{5}-\left(\sigma_{m}+\sigma_{p}+\sigma_{m p}+\phi+\mu_{h}\right) x_{6} \\
& \frac{d x_{7}}{d t}=f_{7}=\alpha \beta_{v}\left(x_{2}+\delta x_{6}\right)\left(1-x_{7}-x_{8}\right)-\left(k_{v}+\mu_{v}\right) x_{7} \\
& \frac{d x_{8}}{d t}=f_{8}=k_{v} x_{7}-\mu_{v} x_{8}
\end{aligned}
$$

It can be shown that system (3.39) has the right and left eigenvectors given by

$w=\left(\frac{D_{1}}{k_{m}} w_{2}, w_{2}, 0,0,0,0, \frac{\alpha \beta_{v}}{\left(k_{v}+\mu_{v}\right)} w_{2}, \frac{\alpha \beta_{v} k_{v}}{\mu_{v}\left(k_{v}+\mu_{v}\right)} w_{2}\right)^{T}$

and

$$
\bar{v}=\left(\frac{k_{m}}{D_{0}} \bar{v}_{2}, \bar{v}_{2}, 0,0, \frac{\alpha^{2} \beta_{v} \delta \beta(H)^{*} k_{m} k_{v} k_{m p}}{\mu_{v}\left(k_{v}+\mu_{v}\right) D_{0} D_{4} D_{5}} \bar{v}_{2}, \frac{\alpha^{2} \beta_{v} \delta \beta(H)^{*} k_{m} k_{v}}{\mu_{v}\left(k_{v}+\mu_{v}\right) D_{0} D_{5}} \bar{v}_{2}, \frac{\alpha \beta(H)^{*} k_{m} k_{v}}{\mu_{v}\left(k_{v}+\mu_{v}\right) D_{0}} \bar{v}_{2}, \frac{\alpha \beta(H)^{*} k_{m}}{\mu_{v} D_{0}} \bar{v}_{2}\right)
$$

where $w_{2}>0$ and $\bar{v}_{2}>0$ are free right and left eigenvector.

\section{Computation of the coefficient $a$ and $b$}


For system (3.43), the associated non-zero partial derivatives of (3.38) at $P_{m p f}$ can be shown to be

$$
\frac{\partial^{2} f_{1}}{\partial x_{1} \partial x_{8}}=\frac{\partial^{2} f_{1}}{\partial x_{2} \partial x_{8}}=-\alpha \beta(H)^{*}, \frac{\partial^{2} f_{7}}{\partial x_{2} \partial x_{7}}=\frac{\partial^{2} f_{7}}{\partial x_{2} \partial x_{8}}=-\alpha \beta_{v}, \frac{\partial^{2} f_{1}}{\partial x_{8} \partial \beta(H)}=\alpha
$$

From (3.41), it follows that

$$
\begin{aligned}
& a=-2 \alpha\left(\bar{v}_{1} w_{1} w_{8} \beta(H)^{*}+\bar{v}_{1} w_{2} w_{8} \beta(H)^{*}+\bar{v}_{7} w_{2} w_{7} \beta_{v}+\bar{v}_{7} w_{2} w_{8} \beta_{v}\right)<0 \\
& b=\bar{v}_{1} w_{8} \alpha>0
\end{aligned}
$$

\subsection{The Effect of Sanitation on Disease Transmission Dynamics}

The aim and ultimate goal of a public health worker is to change the transmission dynamics of a disease in such a way that if an infected individual enters into a community he/she will not trigger an epidemic in the community. Mathematically, it is reasonable to assume that if

$R_{0}<1$, then

$\frac{d I}{d t}<0$

For malaria, intervention can be in the following ways: (i) Treatment using anti-malaria drugs

(ii) Reducing the rate of contact between susceptible individuals and infected mosquitoes and viceversa. The latter can be achieved through sleeping under the mosquito treated nets, clean

environment (maintaining high level of sanitation), etc. The system of equations in (2.3) introduces the functions $\beta(H)$ and $c(H)$ to describe the effect of sanitation on the malaria and pneumonia transmission respectively.

The functions $\beta(H)$ and $c(H)$ in equations (2.4) and (2.5) predicts that malaria and pneumonia transmissions are reduced proportionally to the improvement of sanitation conditions. Using the functions defined in (2.4) and (2.5), a required level of sanitation to prevent the outbreak and low transmission of malaria and pneumonia respectively are established as follows:

If $R_{m}<1$

$$
\begin{aligned}
& \sqrt{\frac{\alpha^{2} \beta_{v} \beta(H) k_{m} k_{v} \Lambda_{h} \Lambda_{v}}{\mu_{h} \mu_{v}^{2}\left(k_{v}+\mu_{v}\right)\left(k_{m}+\pi+\mu_{h}\right)\left(\sigma_{m}+\pi+\mu_{h}\right)}}<1 \\
& \beta(H)<\frac{\mu_{h} \mu_{v}^{2}\left(k_{v}+\mu_{v}\right)\left(k_{m}+\pi+\mu_{h}\right)\left(\sigma_{m}+\pi+\mu_{h}\right)}{\alpha^{2} \beta_{v} k_{m} k_{v} \Lambda_{h} \Lambda_{v}} \\
& \beta_{\text {max }}-\gamma H<\frac{\mu_{h} \mu_{v}{ }^{2}\left(k_{v}+\mu_{v}\right)\left(k_{m}+\pi+\mu_{h}\right)\left(\sigma_{m}+\pi+\mu_{h}\right)}{\alpha^{2} \beta_{v} k_{m} k_{v} \Lambda_{h} \Lambda_{v}}
\end{aligned}
$$

Thus, the level of sanitation required for malaria reduction / eradication is

$$
H>\frac{1}{\gamma}\left[\beta_{\max }-\frac{\mu_{h} \mu_{v}^{2}\left(k_{v}+\mu_{v}\right)\left(k_{m}+\pi+\mu_{h}\right)\left(\sigma_{m}+\pi+\mu_{h}\right)}{\alpha^{2} \beta_{v} k_{m} k_{v} \Lambda_{h} \Lambda_{v}}\right]
$$

Also, if $R_{p}<1$

$$
\begin{aligned}
& c(H)<\frac{\left(\sigma_{p}+\tau+\mu_{h}\right)\left(k_{p}+\tau+V_{p}+\mu_{h}\right) \Lambda_{h}}{\mu_{h} k_{p} \beta_{p}} \\
& c_{\text {max }}-\gamma H<\frac{\left(\sigma_{p}+\tau+\mu_{h}\right)\left(k_{p}+\tau+V_{p}+\mu_{h}\right) \Lambda_{h}}{\mu_{h} k_{p} \beta_{p}}
\end{aligned}
$$

Thus, the level of sanitation required pneumonia reduction / eradication is

$H>\frac{1}{\gamma}\left[c_{\max }-\frac{\left(\sigma_{p}+\tau+\mu_{h}\right)\left(k_{p}+\tau+V_{p}+\mu_{h}\right) \Lambda_{h}}{\mu_{h} k_{p} \beta_{p}}\right]$

\subsection{Application of Optimal Control to the Co-infection of Malaria-Pneumonia}


The main objective of this study is to suggest possible(s) optimal method of reducing/minimizing malaria and pneumonia transmission. Many mathematical model already exist describing malaria infection or pneumonia infection but the best control for malaria infection, pneumonia infection and the co-infection of both diseases still remain a subject of debate. Previous mathematical models have considered treatment, sleeping under mosquito treated bedside nets (MTBN) and the use of insecticide coil as controls. However, these have their limitations. The insecticides used for treating bedside nets is lethal to the mosquitoes, insects and also repels the mosquitoes, thus reducing the number of mosquitoes who attempt to feed on people in the sleeping areas with the nets [2]. However, the mosquitoes can still feed on humans outside these protective areas, hence; Agusto et al [2] included the spraying of insecticides in their model. The latter control by Agusto et al [2] will be more effective in a closed area, hence, the inclusion of sanitation as control in the present work.

The preventive and treatment control for pneumonia includes vaccination, environmental measures and appropriate treatment of other health problems [32]. It is believed that, if appropriate preventive measures were instituted globally, mortality among children could be reduced by 400,000; and, if proper treatment were universally available, childhood deaths could be decreased by another 600,000 [37].

\subsection{Formulation of Optimal Control Model for the Co-infection of Malaria-Pneumonia with Mass Action Incidence}

We now introduce into system (2.3) time dependent preventive measures $\left(u_{1}(t), u_{2}(t), u_{3}(t), u_{4}(t)\right)$ and treatment efforts $\left(u_{5}(t), u_{6}(t), u_{7}(t)\right)$ as controls to curtail the spread of malaria and pneumonia infection. Thus, system (2.3) becomes

$$
\left.\begin{array}{l}
\begin{array}{c}
S_{h}{ }^{\prime}(t)=\Lambda_{h}-\left(1-u_{1}-u_{2}-u_{3}\right) \alpha \beta(H) I_{v} S_{h} \\
-\left(1-u_{3}-u_{4}\right) \beta_{p} c(H)\left(I_{p}+k I_{m p}\right) S_{h}-\mu_{h} S_{h} \\
+u_{5} I_{m}+u_{5} E_{m}+u_{6} I_{p}+u_{6} E_{p}+u_{4} E_{p}+u_{7} I_{m p}+u_{7} E_{m p} \\
E_{m}{ }^{\prime}(t)=\left(1-u_{1}-u_{2}-u_{3}\right) \alpha \beta(H) I_{v} S_{h}-\left(k_{m}+u_{5}+\mu_{h}\right) E_{m}
\end{array} \\
I_{m}{ }^{\prime}(t)=k_{m} E_{m}-\left(1-u_{4}\right) \vartheta \beta_{p} c(H)\left(I_{p}+k I_{m p}\right) I_{m}-\left(\sigma_{m}+u_{5}+\mu_{h}\right) I_{m} \\
E_{p}{ }^{\prime}(t)=\left(1-u_{3}-u_{4}\right) \beta_{p} c(H)\left(I_{p}+k I_{m p}\right) S_{h}-\left(k_{p}+u_{4}+u_{6}+\mu_{h}\right) E_{p} \\
I_{p}{ }^{\prime}(t)=k_{p} E_{p}-\left(1-u_{1}-u_{2}-u_{3}\right) \varepsilon \alpha \beta(H) I_{v} I_{p}-\left(\sigma_{p}+u_{6}+\mu_{h}\right) I_{p} \\
E_{m p}{ }^{\prime}(t)=\left(1-u_{1}-u_{2}-u_{3}\right) \varepsilon \alpha \beta(H) I_{v} I_{p}+\left(1-u_{4}\right) \vartheta \beta_{p} c(H)\left(I_{p}+k I_{m p}\right) I_{m} \\
\quad-\left(k_{m p}+u_{7}+\mu_{h}\right) E_{m p}
\end{array}\right\} \text { (3.48) }
$$


The following cases are considered:

Case 1: The optimal control strategy for malaria transmission model

Case 2: The optimal control strategy for pneumonia transmission model

Case 3: The optimal control strategy for the co-infection of malaria and pneumonia disease transmission model

\subsection{The Optimal Control Strategy for Malaria Transmission Model Case 1}

Here, the formulation of the optimal control problem, analysis of the optimal control problem, adjoint conditions, optimality conditions and the optimality system for the malaria model are considered. Let $I_{p}(t)=I_{m p}(t)=E_{p}(t)=E_{m p}(t)=0$ in model system (3.48) gives the following system of equations

$$
\begin{aligned}
& S_{h}{ }^{\prime}(t)=\Lambda_{h}-\left(1-u_{1}-u_{2}-u_{3}\right) \alpha \beta(H) I_{v} S_{h}-\mu_{h} S_{h}+u_{5} I_{m}+u_{5} E_{m} \\
& E_{m}{ }^{\prime}(t)=\left(1-u_{1}-u_{2}-u_{3}\right) \alpha \beta(H) I_{v} S_{h}-\left(k_{m}+u_{5}+\mu_{h}\right) E_{m} \\
& I_{m}^{\prime}(t)=k_{m} E_{m}-\left(\sigma_{m}+u_{5}+\mu_{h}\right) I_{m} \\
& S_{v}^{\prime}(t)=\Lambda_{v}-\left(1-u_{1}-u_{2}-u_{3}\right) \alpha \beta_{v} I_{m} S_{v}-\left(u_{1}+u_{2}+u_{3}+\mu_{v}\right) S_{v} \\
& E_{v}^{\prime}(t)=\left(1-u_{1}-u_{2}-u_{3}\right) \alpha \beta_{v} I_{m} S_{v}-\left(k_{v}+u_{1}+u_{2}+u_{3}+\mu_{v}\right) E_{v} \\
& I_{v}^{\prime}(t)=k_{v} E_{v}-\left(u_{1}+u_{2}+u_{3}+\mu_{v}\right) I_{v}
\end{aligned}
$$

The controls $u$ in (3.49) is defined to be $u \in[0,1]$, where $u$ ranges from no control $(u=0)$ to maximum control $(u=1)$. Note that $u_{1}, u_{2}, u_{3}, u_{5} \in u$.

The main objective of this research is to find the optimal control strategy $u$ throughout the length of $0 \leq t \leq t_{f}$ such that the numbers of infected humans $I_{m}$ and infected vectors are minimized while minimizing the cost of control $u$. Thus, the objective function is

$J\left(u_{1}, u_{2}, u_{3}, u_{5}\right)=\int_{0}^{t f}\left(M I_{m}+N I_{v}+m_{1} u_{1}{ }^{2}+m_{2} u_{2}{ }^{2}+m_{3} u_{3}{ }^{2}+m_{5} u_{5}{ }^{2}\right) d t$

where coefficients $M, m_{1}, m_{2}, m_{3}$ and $m_{5}$ are positive weights to balance the factors. Thus, we seek an optimal control $u^{*}=\left\{u_{1}{ }^{*}, u_{2}{ }^{*}, u_{3}{ }^{*}, u_{5}{ }^{*}\right\}$ such that

$J\left(u_{1}{ }^{*}, u_{2}{ }^{*}, u_{3}{ }^{*}, u_{5}{ }^{*}\right)=\min _{u_{1}, u_{2}, u_{3}, u_{5}}\left\{\left.J\left(u_{1}, u_{2}, u_{3}, u_{5}\right)\right|_{u_{1}, u_{2}, u_{3}, u_{5}} \in u\right\}$

where

$u=\left\{\left.\left(u_{1}, u_{2}, u_{3}, u_{5}\right)\right|_{u_{1}, u_{2}, u_{3}, u_{5}}:\left[0, t_{f}\right] \rightarrow[0,1]\right\}$

is Lebesgue measurable and convex on $u$, then there exist an optimal control $u$ satisfying the conditions in Appendix A.2

Since there exist an optimal control for minimizing the functional (3.50) subject to system of equations (3.49), the Pontryagins' Maximum Principle [16] is used to derive necessary conditions for this optimal control. The Hamiltonian is defined as follows:

$\bar{H}=M I_{m}+N I_{v}+m_{1} u_{1}^{2}+m_{2} u_{2}^{2}+m_{3} u_{3}^{2}+m_{5} u_{5}^{2}+\lambda_{1}\left[\Lambda_{h}-\left(1-u_{1}-u_{2}-\right.\right.$ $\left.\left.u_{3}\right) \alpha \beta(H) I_{v} S_{h}-\mu_{h} S_{h}+u_{5} I_{m}+u_{5} E_{m}\right]+\lambda_{2}\left[\left(1-u_{1}-u_{2}-u_{3}\right) \alpha \beta(H) I_{v} S_{h}-\left(k_{m}+u_{5}+\right.\right.$ $\left.\left.\mu_{h}\right) E_{m}\right]+\lambda_{3}\left[k_{m} E_{m}-\left(\sigma_{m}+u_{5}+\mu_{h}\right) I_{m}\right]+\lambda_{4}\left[\Lambda_{v}-\left(1-u_{1}-u_{2}-u_{3}\right) \alpha \beta_{v} I_{m} S_{v}-\right.$ $\left.\left(u_{1}+u_{2}+u_{3}+\mu_{v}\right) S_{v}\right]+\lambda_{5}\left[\left(1-u_{1}-u_{2}-u_{3}\right) \alpha \beta_{v} I_{m} S_{v}-\left(k_{v}+u_{1}+u_{2}+u_{3}+\mu_{v}\right) E_{v}\right]+$ $\lambda_{6}\left[k_{v} E_{v}-\left(u_{1}+u_{2}+u_{3}+\mu_{v}\right) I_{v}\right]$

where $\lambda_{1}, \lambda_{2}, \lambda_{3}, \lambda_{4}, \lambda_{5}$ and $\lambda_{6}$ are the adjoint variables or co-state variables.

In order to attach the system of ordinary differential equation in (3.49) on to the objective function in (3.50), the adjoint functions (or co-state variables) were used. The Pontryagins' Maximum Principle gives the necessary conditions that the adjoint functions must satisfy. Thus, the differential equations satisfied by system (3.49) are:

$\frac{d \lambda_{1}}{d t}=-\frac{\partial \bar{H}}{\partial S_{h}}=\left(\lambda_{1}-\lambda_{2}\right)\left(1-u_{1}-u_{2}-u_{3}\right) \alpha \beta(H) I_{v}+\lambda_{1} \mu_{h}$
$\frac{d \lambda_{2}}{d t}=-\frac{\partial \bar{H}}{\partial E_{m}}=\lambda_{2}\left(k_{m}+u_{5}+\mu_{h}\right)-\lambda_{1} u_{5}-\lambda_{3} k_{m}$ 


$$
\begin{aligned}
& \frac{d \lambda_{3}}{d t}=-\frac{\partial \bar{H}}{\partial I_{m}}=\left(\lambda_{4}-\lambda_{5}\right)\left(1-u_{1}-u_{2}-u_{3}\right) \alpha \beta_{v} S_{v}+\lambda_{3}\left(\sigma_{m}+u_{5}+\mu_{h}\right)-\lambda_{1} u_{5}-M \\
& \frac{d \lambda_{4}}{d t}=-\frac{\partial \bar{H}}{\partial S_{v}}=\left(\lambda_{4}-\lambda_{5}\right)\left(1-u_{1}-u_{2}-u_{3}\right) \alpha \beta_{v} I_{m}+\lambda_{4}\left(u_{1}+u_{2}+u_{3}+\mu_{v}\right) \\
& \frac{d \lambda_{5}}{d t}=-\frac{\partial \bar{H}}{\partial E_{v}}=\lambda_{5}\left(k_{v}+u_{1}+u_{2}+u_{3}+\mu_{v}\right)-\lambda_{6} k_{v} \\
& \frac{d \lambda_{6}}{d t}=-\frac{\partial \bar{H}}{\partial I_{v}}=\left(\lambda_{1}-\lambda_{2}\right)\left(1-u_{1}-u_{2}-u_{3}\right) \alpha \beta(H) S_{h}+\lambda_{6}\left(u_{1}+u_{2}+u_{3}+\mu_{v}\right)-N
\end{aligned}
$$

with the boundary conditions (or Transversality conditions) at the final time, $t_{f}$ :

$\lambda_{1}\left(t_{f}\right)=0, \lambda_{2}\left(t_{f}\right)=0, \lambda_{3}\left(t_{f}\right)=0, \lambda_{4}\left(t_{f}\right)=0, \lambda_{5}\left(t_{f}\right)=0, \lambda_{6}\left(t_{f}\right)=0$

The Hamiltonian in (3.53) is minimized with respect to the controls $u_{1}, u_{2}, u_{3}$ and $u_{5}$ separately in order to obtain the optimal value of $u_{1}{ }^{*}, u_{2}{ }^{*}, u_{3}{ }^{*}, u_{5}{ }^{*}$. At these controls values, the maximum Hamiltonian is obtained. The derivative of the Hamiltonian with respect to $u_{1}, u_{2}, u_{3}$ and $u_{5}$ is thus zero, since at the absolute minimum or maximum the slope of a function is zero. Thus,

$u_{1}=\frac{\left(\lambda_{2}-\lambda_{1}\right) \alpha \beta(H) I_{v} S_{h}+\left(\lambda_{5}-\lambda_{4}\right) \alpha \beta_{v} I_{m} S_{v}+\lambda_{5} E_{v}+\lambda_{6} I_{v}+\lambda_{4} S_{v}}{2 m_{1}}$

$u_{2}=\frac{\left(\lambda_{2}-\lambda_{1}\right) \alpha \beta(H) I_{v} S_{h}+\left(\lambda_{5}-\lambda_{4}\right) \alpha \beta_{v} I_{m} S_{v}+\lambda_{5} E_{v}+\lambda_{6} I_{v}+\lambda_{4} S_{v}}{2 m_{2}}$

$u_{3}=\frac{\left(\lambda_{2}-\lambda_{1}\right) \alpha \beta(H) I_{v} S_{h}+\left(\lambda_{5}-\lambda_{4}\right) \alpha \beta_{v} I_{m} S_{v}+\lambda_{5} E_{v}+\lambda_{6} I_{v}+\lambda_{4} S_{v}}{2 m_{3}}$

$u_{5}=\frac{\left(\lambda_{3}-\lambda_{1}\right) I_{m}+\left(\lambda_{2}-\lambda_{1}\right) E_{m}}{2 m_{5}}$

At the absolute minimum $u=u^{*}$, therefore the optimality conditions are

$$
\left.\begin{array}{l}
u_{1}{ }^{*}=\min \left\{1, \max \left(0, u_{1}\right)\right\} \\
u_{2}{ }^{*}=\min \left\{1, \max \left(0, u_{2}\right)\right\} \\
u_{3}{ }^{*}=\min \left\{1, \max \left(0, u_{3}\right)\right\} \\
u_{5}{ }^{*}=\min \left\{1, \max \left(0, u_{5}\right)\right\}
\end{array}\right\}
$$

\subsection{The Optimal Control Strategy for Pneumonia Transmission Model Case 2}

If $E_{m}(t)=I_{m}(t)=E_{m p}(t)=I_{m p}(t)=E_{v}(t)=I_{v}(t)=0$ in model system (3.48) gives the following system of equations

$$
\left.\begin{array}{l}
S_{h}{ }^{\prime}(t)=\Lambda_{h}-\left(1-u_{3}-u_{4}\right) \beta_{p} c(H) I_{p} S_{h}-\mu_{h} S_{h}+u_{6} E_{p}+u_{6} I_{p}+u_{4} E_{p} \\
E_{p}{ }^{\prime}(t)=\left(1-u_{3}-u_{4}\right) \beta_{p} c(H) I_{p} S_{h}-\left(k_{p}+u_{6}+u_{4}+\mu_{h}\right) E_{p} \\
I_{p}{ }^{\prime}(t)=k_{p} E_{p}-\left(\sigma_{p}+u_{6}+\mu_{h}\right) I_{p} \\
S_{v}{ }^{\prime}(t)=\Lambda_{v}-\left(u_{1}+u_{2}+u_{3}+\mu_{v}\right) S_{v}
\end{array}\right\}
$$

Observe that the fourth differential equation in (3.61) is independent of the first three differential equations; hence, system (3.67) can then be written as

$$
\left.\begin{array}{l}
S_{h}{ }^{\prime}(t)=\Lambda_{h}-\left(1-u_{3}-u_{4}\right) \beta_{p} c(H) I_{p} S_{h}-\mu_{h} S_{h}+u_{6} E_{p}+u_{6} I_{p}+u_{4} E_{p} \\
E_{p}{ }^{\prime}(t)=\left(1-u_{3}-u_{4}\right) \beta_{p} c(H) I_{p} S_{h}-\left(k_{p}+u_{6}+u_{4}+\mu_{h}\right) E_{p} \\
I_{p}{ }^{\prime}(t)=k_{p} E_{p}-\left(\sigma_{p}+u_{6}+\mu_{h}\right) I_{p}
\end{array}\right\}
$$

where the controls $u_{3}, u_{4}$ and $u_{6}$ retain their original meaning as defined in system (3.48) The controls $u$ in (3.62) is defined to be $u \in[0,1]$, for $u_{3}, u_{4}, u_{6} \in u$

The objective is such that the numbers of infected humans with pneumonia $I_{p}$ is minimized while minimizing the cost of control $u$. Hence 
$J\left(u_{3}, u_{4}, u_{6}\right)=\int_{0}^{t f}\left(D I_{p}+m_{3} u_{3}^{2}+m_{4} u_{4}^{2}+m_{6} u_{6}^{2}\right) d t$

where coefficients $D, m_{3}, m_{4}$ and $m_{6}$ are positive weights to balance the factors.

Thus, we seek an optimal control $u^{*}=\left\{u_{3}{ }^{*}, u_{4}{ }^{*}, u_{6}{ }^{*}\right\}$ such that

$J\left(u_{3}{ }^{*}, u_{4}{ }^{*}, u_{6}{ }^{*}\right)=\min _{u_{2}, u_{4}, u_{6}}\left\{\left.J\left(u_{2}, u_{4}, u_{6}\right)\right|_{u_{2}, u_{4}, u_{6}} \in u\right\}$

where

$u=\left\{\left.\left(u_{2}, u_{4}, u_{6}\right)\right|_{u_{2}, u_{4}, u_{6}}:\left[0, t_{f}\right] \rightarrow[0,1]\right\}$

is Lebesgue measurable and convex on $u$, then there exist an optimal control $u$ satisfying the conditions in Appendix A.2. We define an Hamiltonian as follows:

$\bar{H}=D I_{p}+m_{3} u_{3}^{2}+m_{4} u_{4}^{2}+m_{6} u_{6}^{2}+\lambda_{1}\left[\Lambda_{h}-\left(1-u_{3}-u_{4}\right) \beta_{p} c(H) I_{p} S_{h}-\mu_{h} S_{h}+u_{6} E_{p}+\right.$ $\left.u_{6} I_{p}+u_{4} E_{p}\right]+\lambda_{2}\left[\left(1-u_{3}-u_{4}\right) \beta_{p} c(H) I_{p} S_{h}-\left(k_{p}+u_{6}+u_{4}+\mu_{h}\right) E_{p}\right]+\lambda_{3}\left[k_{p} E_{p}-\right.$ $\left.\left(\sigma_{p}+u_{6}+\mu_{h}\right) I_{p}\right]$

with $\lambda_{1}, \lambda_{2}, \lambda_{3}$ defined as the adjoint variables or co-state variables and obtained as

$$
\begin{aligned}
\frac{d \lambda_{1}}{d t} & =-\frac{\partial \bar{H}}{\partial S_{h}}=\left(\lambda_{1}-\lambda_{2}\right)\left(1-u_{3}-u_{4}\right) \beta_{p} c(H) I_{p}+\lambda_{1} \mu_{h} \\
\frac{d \lambda_{2}}{d t} & =-\frac{\partial \bar{H}}{\partial E_{p}}=\lambda_{2}\left(k_{p}+u_{4}+u_{6}+\mu_{h}\right)-\left(u_{4}+u_{6}\right) \lambda_{1}-\lambda_{3} k_{p} \\
\frac{d \lambda_{3}}{d t} & =-\frac{\partial \bar{H}}{\partial I_{p}}=\left(\lambda_{1}-\lambda_{2}\right)\left(1-u_{3}-u_{4}\right) \beta_{p} c(H) S_{h}+\lambda_{3}\left(\sigma_{p}+u_{6}+\mu_{h}\right)-D
\end{aligned}
$$

with the boundary conditions (or Transversality conditions) at the final time, $t_{f}$ :

$\lambda_{1}\left(t_{f}\right)=0, \lambda_{2}\left(t_{f}\right)=0, \lambda_{3}\left(t_{f}\right)=0$

The Hamiltonian in (3.72) is minimized with respect to the controls $u_{3}, u_{4}$ and $u_{6}$ separately in order to obtain the optimal value of $u_{3}{ }^{*}, u_{4}{ }^{*}, u_{6}{ }^{*}$. Thus,

$u_{3}=\frac{\left(\lambda_{2}-\lambda_{1}\right) \beta_{p} c(H) I_{p} S_{h}}{2 m_{3}}$

$u_{4}=\frac{\left(\lambda_{2}-\lambda_{1}\right) \beta_{p} c(H) I_{p} S_{h}+\left(\lambda_{2}-\lambda_{1}\right) E_{p}}{2 m_{4}}$

$u_{6}=\frac{\left(\lambda_{2}-\lambda_{1}\right) E_{p}+\left(\lambda_{3}-\lambda_{1}\right) I_{p}}{2 m_{6}}$

The optimality conditions in this case are

$$
\left.\begin{array}{rl}
u_{3}{ }^{*} & =\min \left\{1, \max \left(0, u_{3}\right)\right\} \\
u_{4}{ }^{*} & =\min \left\{1, \max \left(0, u_{4}\right)\right\} \\
u_{6}{ }^{*} & =\min \left\{1, \max \left(0, u_{6}\right)\right\}
\end{array}\right\}
$$

\subsection{The Optimal Control Model for the Co-infection of Malaria-Pneumonia with Mass Action Incidence Model Case 3}


Recall the system of equations in (3.48) written below as

$$
\begin{gathered}
S_{h}{ }^{\prime}(t)=\Lambda_{h}-\left(1-u_{1}-u_{2}-u_{3}\right) \alpha \beta(H) I_{v} S_{h} \\
\quad-\left(1-u_{3}-u_{4}\right) \beta_{p} c(H)\left(I_{p}+k I_{m p}\right) S_{h}-\mu_{h} S_{h} \\
\quad+u_{5} I_{m}+u_{5} E_{m}+u_{6} I_{p}+u_{6} E_{p}+u_{4} E_{p}+u_{7} I_{m p}+u_{7} E_{m p} \\
E_{m}{ }^{\prime}(t)=\left(1-u_{1}-u_{2}-u_{3}\right) \alpha \beta(H) I_{v} S_{h}-\left(k_{m}+u_{5}+\mu_{h}\right) E_{m} \\
I_{m}{ }^{\prime}(t)=k_{m} E_{m}-\left(1-u_{4}\right) \vartheta \beta_{p} c(H)\left(I_{p}+k I_{m p}\right) I_{m}-\left(\sigma_{m}+u_{5}+\mu_{h}\right) I_{m} \\
E_{p}{ }^{\prime}(t)=\left(1-u_{3}-u_{4}\right) \beta_{p} c(H)\left(I_{p}+k I_{m p}\right) S_{h}-\left(k_{p}+u_{4}+u_{6}+\mu_{h}\right) E_{p} \\
I_{p}{ }^{\prime}(t)=k_{p} E_{p}-\left(1-u_{1}-u_{2}-u_{3}\right) \varepsilon \alpha \beta(H) I_{v} I_{p}-\left(\sigma_{p}+u_{6}+\mu_{h}\right) I_{p} \\
E_{m p}{ }^{\prime}(t)=\left(1-u_{1}-u_{2}-u_{3}\right) \varepsilon \alpha \beta(H) I_{v} I_{p}+\left(1-u_{4}\right) \vartheta \beta_{p} c(H)\left(I_{p}+k I_{m p}\right) I_{m} \\
\quad-\left(k_{m p}+u_{7}+\mu_{h}\right) E_{m p} \\
I_{m p}{ }^{\prime}(t)=k_{m p} E_{m p}-\left(\sigma_{m}+\sigma_{p}+\sigma_{m p}+u_{7}+\mu_{h}\right) I_{m p} \\
S_{v}{ }^{\prime}(t)=\Lambda_{v}-\left(1-u_{1}-u_{2}-u_{3}\right) \alpha \beta_{v}\left(I_{m}+\delta I_{m p}\right) S_{v} \\
\quad-\left(u_{1}+u_{2}+u_{3}+\mu_{v}\right) S_{v} \\
E_{v}{ }^{\prime}(t)=\left(1-u_{1}-u_{2}-u_{3}\right) \alpha \beta_{v}\left(I_{m}+\delta I_{m p}\right) S_{v}-\left(k_{v}+u_{1}+u_{2}+u_{3}+\mu_{v}\right) E_{v} \\
I_{v}{ }^{\prime}(t)=k_{v} E_{v}-\left(u_{1}+u_{2}+u_{3}+\mu_{v}\right) I_{v}
\end{gathered}
$$

where the controls $u_{1}, u_{2}, u_{3}, u_{4}, u_{5}, u_{6}$ and $u_{7}$ retain their original meaning as defined in system (3.48). The objective functional is

$J\left(u_{1}, u_{2}, u_{3}, u_{5}\right)=\int_{0}^{t f}\left(M I_{m}+D I_{p}+Q I_{m p}+N I_{v}+m_{1} u_{1}^{2}+m_{2} u_{2}{ }^{2}+m_{3} u_{3}^{2}+m_{4} u_{4}^{2}+\right.$ $\left.m_{5} u_{5}{ }^{2}+m_{6} u_{6}{ }^{2}+m_{7} u_{7}^{2}\right) d t$

where the coefficients $D, M, N, Q, m_{1}, m_{2}, m_{3}, m_{4}, m_{5}, m_{6}$ and $m_{7}$ are positive weights to balance the factors. Thus, an optimal control $u^{*}=\left\{u_{1}{ }^{*}, u_{2}{ }^{*}, u_{3}{ }^{*}, u_{4}{ }^{*}, u_{5}{ }^{*}, u_{6}{ }^{*}, u_{7}{ }^{*}\right\}$ is sought such that

$J\left(u_{1}{ }^{*}, u_{2}{ }^{*}, u_{3}{ }^{*}, u_{4}{ }^{*}, u_{5}{ }^{*}, u_{6}{ }^{*}, u_{7}{ }^{*}\right)=\min _{u_{1}, u_{2}, u_{3}, u_{5}}\left\{\left.J\left(u_{1}, u_{2}, u_{3}, u_{4}, u_{5}, u_{6}, u_{7}\right)\right|_{u_{1}, u_{2}, u_{3}, u_{5}} \in u\right\}$

where

$u=\left\{\left.\left(u_{1}, u_{2}, u_{3}, u_{4}, u_{5}, u_{6}, u_{7}\right)\right|_{u_{1}, u_{2}, u_{3}, u_{4}, u_{5}, u_{6}, u_{7}}:\left[0, t_{f}\right] \rightarrow[0,1]\right\}$

is Lebesgue measurable and convex on $u$, then there exist an optimal control $u$ satisfying the conditions in section 2.7. The Hamiltonian is defined as

$\bar{H}=M I_{m}+D I_{p}+Q I_{m p}+N I_{v}+m_{1} u_{1}{ }^{2}+m_{2} u_{2}{ }^{2}+m_{3} u_{3}{ }^{2}+m_{4} u_{4}{ }^{2}+m_{5} u_{5}{ }^{2}+m_{6} u_{6}{ }^{2}+$ $m_{7} u_{7}^{2}+\lambda_{1}\left[\Lambda_{h}-\left(1-u_{1}-u_{2}-u_{3}\right) \alpha \beta(H) I_{v} S_{h}-\left(1-u_{3}-u_{4}\right) \beta_{p} c(H)\left(I_{p}+k I_{m p}\right) S_{h}-\right.$ $\left.\mu_{h} S_{h}+u_{5} I_{m}+u_{5} E_{m}+u_{6} I_{p}+u_{6} E_{p}+u_{4} E_{p}+u_{7} I_{m p}+u_{7} E_{m p}\right]+\lambda_{2}\left[\left(1-u_{1}-u_{2}-\right.\right.$ $\left.\left.u_{3}\right) \alpha \beta(H) I_{v} S_{h}-\left(k_{m}+u_{5}+\mu_{h}\right) E_{m}\right]+\lambda_{3}\left[k_{m} E_{m}-\left(1-u_{4}\right) \vartheta \beta_{p} c(H)\left(I_{p}+k I_{m p}\right) I_{m}-\right.$ $\left.\left(\sigma_{m}+u_{5}+\mu_{h}\right) I_{m}\right]+\lambda_{4}\left[\left(1-u_{3}-u_{4}\right) \beta_{p} c(H)\left(I_{p}+k I_{m p}\right) S_{h}-\left(k_{p}+u_{4}+u_{6}+\mu_{h}\right) E_{p}\right]+$ $\lambda_{5}\left[k_{p} E_{p}-\left(1-u_{1}-u_{2}-u_{3}\right) \varepsilon \alpha \beta(H) I_{v} I_{p}-\left(\sigma_{p}+u_{6}+\mu_{h}\right) I_{p}\right]+\lambda_{6}\left[\left(1-u_{1}-u_{2}-\right.\right.$ $\left.\left.u_{3}\right) \varepsilon \alpha \beta(H) I_{v} I_{p}+\left(1-u_{4}\right) \vartheta \beta_{p} c(H)\left(I_{p}+k I_{m p}\right) I_{m}-\left(k_{m p}+u_{7}+\mu_{h}\right) E_{m p}\right]+\lambda_{7}\left[k_{m p} E_{m p}-\right.$ $\left.\left(\sigma_{m}+\sigma_{p}+\sigma_{m p}+u_{7}+\mu_{h}\right) I_{m p}\right]+\lambda_{8}\left[\Lambda_{v}-\left(1-u_{1}-u_{2}-u_{3}\right) \alpha \beta_{v}\left(I_{m}+\delta I_{m p}\right) S_{v}-\right.$ $\left.\left(u_{1}+u_{2}+u_{3}+\mu_{v}\right) S_{v}\right]+\lambda_{9}\left[\left(1-u_{1}-u_{2}-u_{3}\right) \alpha \beta_{v}\left(I_{m}+\delta I_{m p}\right) S_{v}-\left(k_{v}+u_{1}+u_{2}+u_{3}+\right.\right.$ $\left.\left.\mu_{v}\right) E_{v}\right]+\lambda_{10}\left[k_{v} E_{v}-\left(u_{1}+u_{2}+u_{3}+\mu_{v}\right) I_{v}\right]$

with the following adjoint variables (or co-state variables) satisfying the Pontryagin Maximum Principle condition: 


$$
\begin{aligned}
& \left.\frac{d \lambda_{1}}{d t}=-\frac{\partial \bar{H}}{\partial S_{h}}=\left(\lambda_{1}-\lambda_{2}\right)\left(1-u_{1}-u_{2}-u_{3}\right) \alpha \beta(H) I_{v}+\left(\lambda_{1}-\lambda_{4}\right)\left(1-u_{3}-u_{4}\right) \beta_{p} c(H)\left(I_{p}+k I_{m p}\right)+\lambda_{1} \mu_{h}\right) \\
& \frac{d \lambda_{2}}{d t}=-\frac{\partial \bar{H}}{\partial E_{m}}=\lambda_{2}\left(k_{m}+u_{5}+\mu_{h}\right)-\lambda_{1} u_{5}-\lambda_{3} k_{m} \\
& \frac{d \lambda_{3}}{d t}=-\frac{\partial \bar{H}}{\partial I_{m}}=\left(\lambda_{3}-\lambda_{6}\right)\left(1-u_{4}\right) \vartheta \beta_{p} c(H)\left(I_{p}+k I_{m p}\right)+\left(\lambda_{8}-\lambda_{9}\right)\left(1-u_{1}-u_{2}-u_{3}\right) \alpha \beta_{v} S_{v} \\
& +\lambda_{3}\left(\sigma_{m}+u_{5}+\mu_{h}\right)-\lambda_{1} u_{5}-M \\
& \frac{d \lambda_{4}}{d t}=-\frac{\partial \bar{H}}{\partial E_{p}}=\lambda_{4}\left(k_{p}+u_{4}+u_{6}+\mu_{h}\right)-\lambda_{1}\left(u_{4}+u_{6}\right)-\lambda_{5} k_{p} \\
& \frac{d \lambda_{5}}{d t}=-\frac{\partial \bar{H}}{\partial I_{p}}=\left(\lambda_{3}-\lambda_{6}\right)\left(1-u_{4}\right) \vartheta \beta_{p} c(H) I_{m}+\left(\lambda_{5}-\lambda_{6}\right)\left(1-u_{1}-u_{2}-u_{3}\right) \varepsilon \alpha \beta(H) I_{v} \\
& +\left(\lambda_{1}-\lambda_{4}\right)\left(1-u_{3}-u_{4}\right) \beta_{p} c(H) S_{h}+\lambda_{3}\left(\sigma_{p}+u_{6}+\mu_{h}\right)-\lambda_{1} u_{6}-D \\
& \frac{d \lambda_{6}}{d t}=-\frac{\partial \bar{H}}{\partial E_{m p}}=\lambda_{6}\left(k_{m p}+u_{7}+\mu_{h}\right)-\lambda_{1} u_{7}-\lambda_{7} k_{m p} \\
& \frac{d \lambda_{7}}{d t}=-\frac{\partial \bar{H}}{\partial I_{m p}}=\left(\lambda_{1}-\lambda_{4}\right)\left(1-u_{3}-u_{4}\right) \beta_{p} c(H) k S_{h}+\left(\lambda_{3}-\lambda_{6}\right)\left(1-u_{4}\right) \vartheta \beta_{p} k c(H) I_{m} \\
& +\left(\lambda_{8}-\lambda_{9}\right)\left(1-u_{1}-u_{2}-u_{3}\right) \alpha \beta_{v} \delta S_{v}+\lambda_{7}\left(\sigma_{m}+\sigma_{p}+\sigma_{m p}+u_{7}+\mu_{h}\right)-\lambda_{1} u_{7}-Q \\
& \frac{d \lambda_{8}}{d t}=-\frac{\partial \bar{H}}{\partial S_{v}}=\left(\lambda_{8}-\lambda_{9}\right)\left(1-u_{1}-u_{2}-u_{3}\right) \alpha \beta_{v}\left(I_{m}+\delta I_{m p}\right)+\lambda_{8}\left(u_{1}+u_{2}+u_{3}+\mu_{v}\right) \\
& \frac{d \lambda_{9}}{d t}=-\frac{\partial \bar{H}}{\partial E_{v}}=\lambda_{9}\left(k_{v}+u_{1}+u_{2}+u_{3}+\mu_{v}\right)-\lambda_{10} k_{v} \\
& \frac{d \lambda_{10}}{d t}=-\frac{\partial \bar{H}}{\partial I_{v}}=\left(\lambda_{1}-\lambda_{2}\right)\left(1-u_{1}-u_{2}-u_{3}\right) \alpha \beta(H) S_{h}+\left(\lambda_{5}-\lambda_{6}\right)\left(1-u_{1}-u_{2}-u_{3}\right) \varepsilon \beta(H) I_{p} \\
& +\lambda_{10}\left(u_{1}+u_{2}+u_{3}+\mu_{v}\right)-N
\end{aligned}
$$

with boundary conditions

$\lambda_{1}\left(t_{f}\right)=\lambda_{2}\left(t_{f}\right)=\lambda_{3}\left(t_{f}\right)=\lambda_{4}\left(t_{f}\right)=\lambda_{5}\left(t_{f}\right)=\lambda_{6}\left(t_{f}\right)=\lambda_{7}\left(t_{f}\right)=\lambda_{8}\left(t_{f}\right)=\lambda_{9}\left(t_{f}\right)=$ $\lambda_{10}\left(t_{f}\right)=0$

The derivative of the Hamiltonian with respect to $u_{1}, u_{2}, u_{3}, u_{4}, u_{5}, u_{6}$ and $u_{7}$ is thus zero, since at the absolute minimum or maximum the slope of a function is zero. Then

$u_{1}$

$=\frac{\left(\lambda_{2}-\lambda_{1}\right) \alpha \beta(H) I_{v} S_{h}+\left(\lambda_{6}-\lambda_{5}\right) \varepsilon \alpha \beta(H) I_{v} I_{p}+\left(\lambda_{9}-\lambda_{8}\right) \alpha \beta_{v}\left(I_{m}+\delta I_{m p}\right) S_{v}+\lambda_{8} S_{v}+\lambda_{9} E_{v}+\lambda_{10} I_{v}}{2 m_{1}}$

$u_{2}$

$=\frac{\left(\lambda_{2}-\lambda_{1}\right) \alpha \beta(H) I_{v} S_{h}+\left(\lambda_{6}-\lambda_{5}\right) \varepsilon \alpha \beta(H) I_{v} I_{p}+\left(\lambda_{9}-\lambda_{8}\right) \alpha \beta_{v}\left(I_{m}+\delta I_{m p}\right) S_{v}+\lambda_{8} S_{v}+\lambda_{9} E_{v}+\lambda_{10} I_{v}}{2 m_{2}}$

$u_{3}$

$=\frac{\left(\lambda_{2}-\lambda_{1}\right) \alpha \beta(H) I_{v} S_{h}+\left(\lambda_{4}-\lambda_{1}\right) \beta_{p} C(H)\left(I_{p}+k I_{m p}\right) S_{h}+\left(\lambda_{6}-\lambda_{5}\right) \varepsilon \alpha \beta(H) I_{v} I_{p}+\left(\lambda_{9}-\lambda_{8}\right) \alpha \beta_{v}\left(I_{m}+\delta I_{m p}\right) S_{v}+\lambda_{8} S_{v}+\lambda_{9} E_{v}+\lambda_{10} I_{v}}{2 m_{3}}$

$u_{4}=\frac{\left(\lambda_{4}-\lambda_{1}\right) \beta_{p} c(H)\left(I_{p}+k I_{m p}\right) S_{h}+\left(\lambda_{4}-\lambda_{1}\right) E_{p}+\left(\lambda_{6}-\lambda_{3}\right) \vartheta \beta_{p} c(H)\left(I_{p}+k I_{m p}\right) I_{m}}{2 m_{4}}$

$u_{5}=\frac{\left(\lambda_{3}-\lambda_{1}\right) I_{m}+\left(\lambda_{2}-\lambda_{1}\right) E_{m}}{2 m_{5}}$

$u_{6}=\frac{\left(\lambda_{5}-\lambda_{1}\right) I_{p}+\left(\lambda_{4}-\lambda_{1}\right) E_{p}}{2 m_{6}}$ 
$u_{7}=\frac{\left(\lambda_{7}-\lambda_{1}\right) I_{m p}+\left(\lambda_{6}-\lambda_{1}\right) E_{m p}}{2 m_{7}}$

Therefore the optimality conditions are

$u_{1}^{*}=\min \left\{1, \max \left(0, u_{1}\right)\right\}$

$u_{2}{ }^{*}=\min \left\{1, \max \left(0, u_{2}\right)\right\}$

$u_{3}{ }^{*}=\min \left\{1, \max \left(0, u_{3}\right)\right\}$

$\left.u_{4}{ }^{*}=\min \left\{1, \max \left(0, u_{4}\right)\right\}\right\}$

$u_{5}{ }^{*}=\min \left\{1, \max \left(0, u_{5}\right)\right\}$

$u_{6}{ }^{*}=\min \left\{1, \max \left(0, u_{6}\right)\right\}$

$u_{7}{ }^{*}=\min \left\{1, \max \left(0, u_{7}\right)\right\}$

\subsection{Numerical Results and Discussion}

In this section, we study numerically for the purpose of illustration the malaria only model, pneumonia only model, the malaria-pneumonia co-infection model and their corresponding optimal control models. The numerical computations were performed using MAPEL 18 program with computation times of 5.0s on a windows 7 operating system core i5. The optimal control was obtained by solving the optimality systems for malaria only, pneumonia only and malariapneumonia co-infection models respectively. An iterative forward and backward finite difference scheme are used to solve the optimality system; the forward finite difference was used to solve the state equations and the backward finite difference scheme was used to solve the co-state (adjoint) equations using the current iterations solutions of the state equations because of the transversality conditions. Then the controls are updated by using a convex combination of the previous controls and the value from the characterization (3.60), (3.72) and (3.78). Thus, the process is repeated and the iterations are stopped at the final time $t_{f}$.

The analytical results of the study are illustrated by simulating the model systems using values from other literature. The scarcity of data on the malaria-pneumonia co-infection limits our ability to scale our analytical results, however, for the purpose of illustration, other parameter values are assumed to vary within realistic means as given in Table 1

Table 1 Values of Parameters for the Malaria, Pneumonia and Co-infection Model

\section{Parameters}

$k_{m}$

$\beta_{\max }$

$\gamma$

$\beta_{v}$

$\mu_{h}$

$\mu_{v}$

$k_{v}$

$\Lambda_{h}$

$\alpha$

$\sigma_{m}$

$\Lambda_{v}$

$\pi$

$k_{p}$

$c_{\max }$

$\beta_{p}$
Value

$\frac{1}{17}$

0.003

0.009

0.001

0.0000457

0.04

$\frac{1}{18}$

0.00011

0.5

0.01

0.071

0.5

0.5

0.005

0.5
Sources

Assumed

Assumed

[5]

[39]

[5]

Assumed

[4]

Assumed

Assumed

Assumed

Assumed 


$\begin{array}{lll}V_{p} & 0.5 & \text { Assumed } \\ \tau & 0.5 & \text { Assumed } \\ \vartheta & 0.1 & \text { Assumed } \\ \varepsilon & 0.1 & \text { Assumed } \\ \delta & 0.5 & \text { Assumed } \\ \sigma_{m p} & 0.1 & \text { Assumed } \\ \sigma_{p} & 0.01 & \text { Assumed } \\ k & 0.5 & \text { Assumed } \\ k_{m p} & 0.5 & \text { Assumed }\end{array}$

It was observed that as the biting rate, $\boldsymbol{\alpha}$ of the infected mosquitoes increase, there is a rapid decline in the number of susceptible human population as shown in Figure 1. This is expected from the result of the sensitivity analysis on the malaria model in case 1 that the biting rates of the mosquitoes, $\boldsymbol{\alpha}$, is the most sensitive of all the parameters in the malaria model being considered. This explains the rapid reduction in the number of susceptible individuals in the population as the rate of mosquito biting rates $\boldsymbol{\alpha}$ increases. This result is also in perfect agreement with the result obtained in Tumwiine et al [34] that reduction in contact between infected mosquitoes and humans was necessary in bringing the basic reproduction number below unity. The effect of treatment rate $\pi$ using anti-malaria drugs on infected individual with malaria were also considered and it can be seen from Figure 2 that as treatment rate $\pi$ of malaria infected individuals increases, the proportion of infected humans decreases because treated individual leave the infected class and move to the susceptible class upon recovery from the malaria disease, a result which is in agreement with those obtained in [34] that there was need for effective drugs to reduce the spread of malaria disease.

Figure 3 is the backward bifurcation diagram for the malaria model where an endemic equilibrium co-exist (dotted lines) with the disease-free equilibrium. The backward bifurcation phenomenon explains that for total malaria eradication in the population $\boldsymbol{R}_{\boldsymbol{m}}<\mathbf{1}$ is not sufficient rather $\boldsymbol{R}_{\boldsymbol{m}}$ must be brought below certain threshold $\boldsymbol{R}_{\boldsymbol{c}}$ such that $\boldsymbol{R}_{\boldsymbol{m}}<\boldsymbol{R}_{\boldsymbol{c}}<\mathbf{1} . \boldsymbol{R}_{\boldsymbol{m}}$ is defined here as the basic reproduction number for malaria. The impact of backward bifurcation on malaria transmission is to ensure that proper diagnosis are carried out on patients rather than treating patients based on symptoms and individuals who had being diagnosed to have malaria should go through complete treatment. From Figure 4, it was observed that if the contact rate $c=0$ between susceptible and an infected individual with pneumonia, it was noticed that the number of susceptible human population increases considerably, however, as the rate of contact $c$ increases, there was a sharp decline in the number of susceptible human population into the exposed human to pneumonia and subsequently progress to infected pneumonia class. This affirms the result of the sensitivity analysis that the contact rates $c$ between susceptible individuals and infected individual is the most sensitive parameter. This claim was also supported by Mandell et al [25]. Therefore, reduction in effective contacts between susceptible and an infected individual is recommended through good sanitation among other measures.

The effect of treatment rate $\tau$ was considered in Figure 5. It was observed that the number of treated individuals with pneumonia decreases as the treatment rate $\tau$ using anti - pneumonia drugs on the infected individuals in the population increases. Figure 6 explains the role vaccination played in individuals exposed to pneumonia disease. The anti-pneumonia vaccines prevent exposed individuals from progressing to the infectious class. Increase in the vaccination rate, $\boldsymbol{V}_{\boldsymbol{p}}$, result in decrease in the number of exposed individuals. This result is supported by the claim of ([30], [32]).

The pneumonia model discussed in case 2 exhibited a forward bifurcation which suggest that it is sufficient to reduce $\boldsymbol{R}_{\boldsymbol{p}}$ below unity to guarantee total eradication of pneumonia disease in 
the population while the disease persist in the population if $\boldsymbol{R}_{\boldsymbol{p}}$ is greater than unity as shown in Figure 7. As established in the malaria model and pneumonia model of this study, that increase in treatment rate of malaria and pneumonia diseases reduce the transmission rates of the two diseases, hence, Figure 8 also establish that using the combination of anti-malaria and anti-pneumonia drugs reduces the co-infection of malaria - pneumonia diseases. The result of [22] affirm our assertion that individual with malaria - pneumonia symptoms overlap are treated with both anti malaria and anti - biotics. The bifurcation diagram for the malaria - pneumonia co - infection is presented in Figure 9. The diagram above is in perfect agreement with our analytical result; that where malaria and pneumonia co - exist the condition $\boldsymbol{R}_{\boldsymbol{m}}<\boldsymbol{R}_{c}<\boldsymbol{R}_{\boldsymbol{p}}<\mathbf{1}$ is required for malaria and pneumonia to be eradicated in the population. Note that $\boldsymbol{R}_{\boldsymbol{m} \boldsymbol{p}}=\boldsymbol{\operatorname { m a x }}\left\{\boldsymbol{R}_{\boldsymbol{m}}, \boldsymbol{R}_{\boldsymbol{p}}\right\}$, where $\boldsymbol{R}_{\boldsymbol{m} \boldsymbol{p}}$ is defined to be the basic reproduction number for co - infection of mlaria and pneumonia.

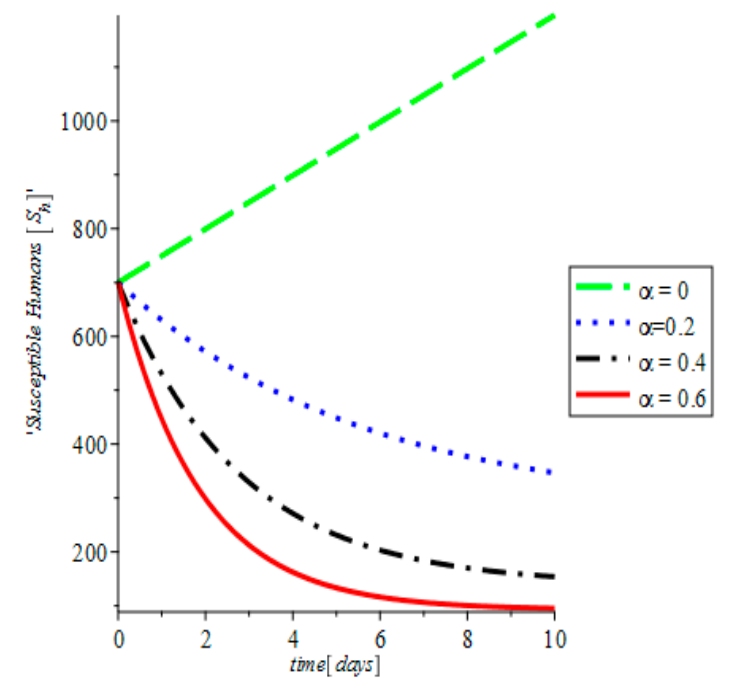

Figure 1: The variation of proportion of Susceptible Human population for different values of $\alpha$

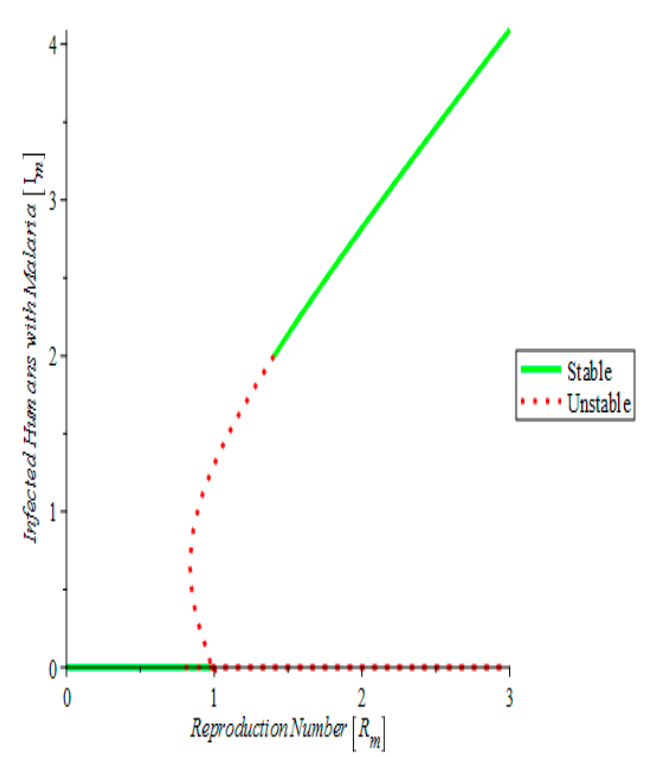

Figure 3: The backward bifurcation diagram for malaria infection

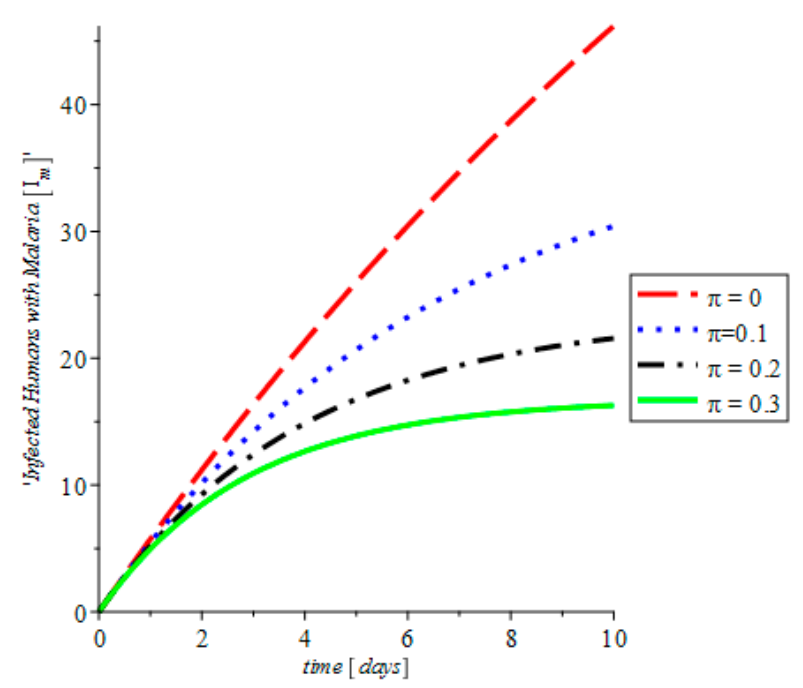

Figure 2: The variation of proportion of malaria infected humanpopulation for different values of $\pi$

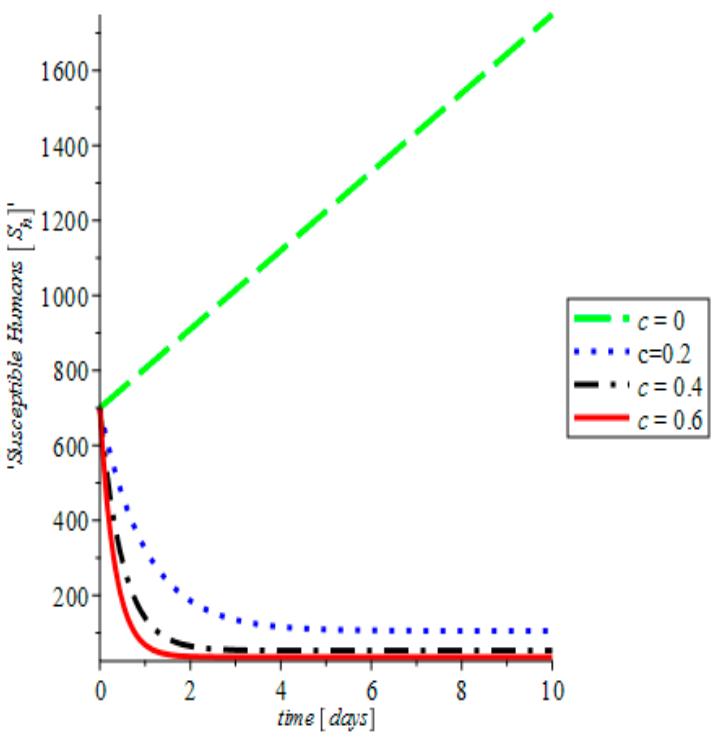

Figure 4: The variation of proportion of susceptible human population for different values of $c$ 


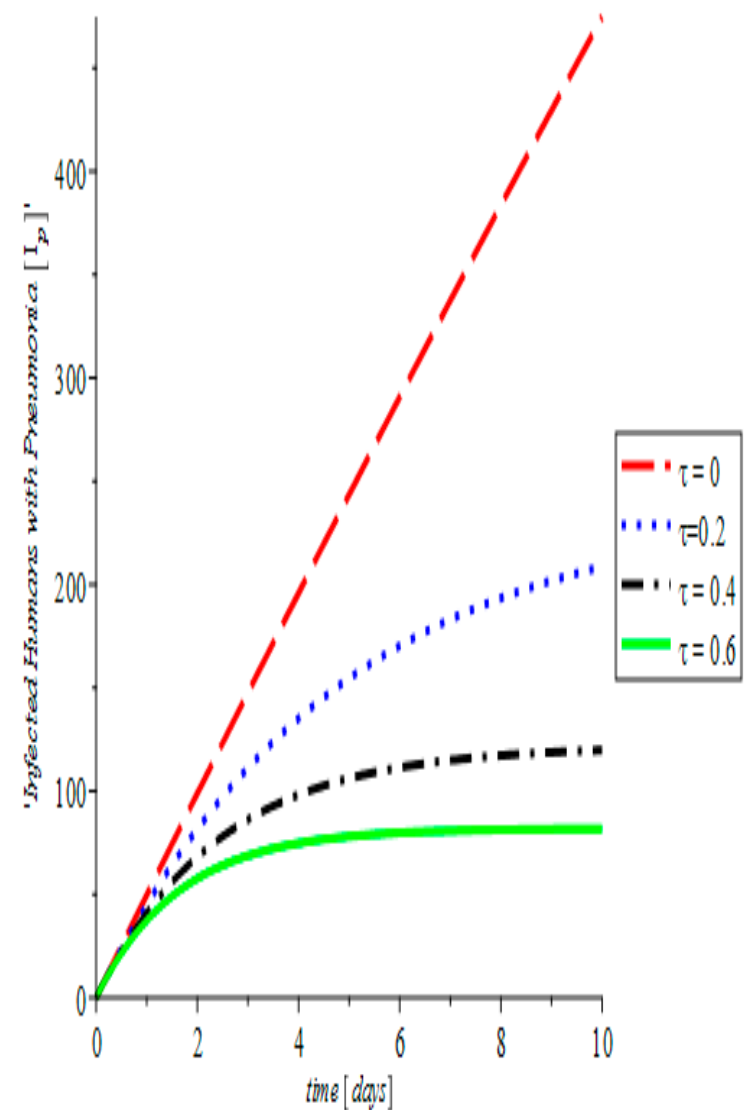

Figure 5: The variation of proportion of susceptible human population for different values of $\tau$

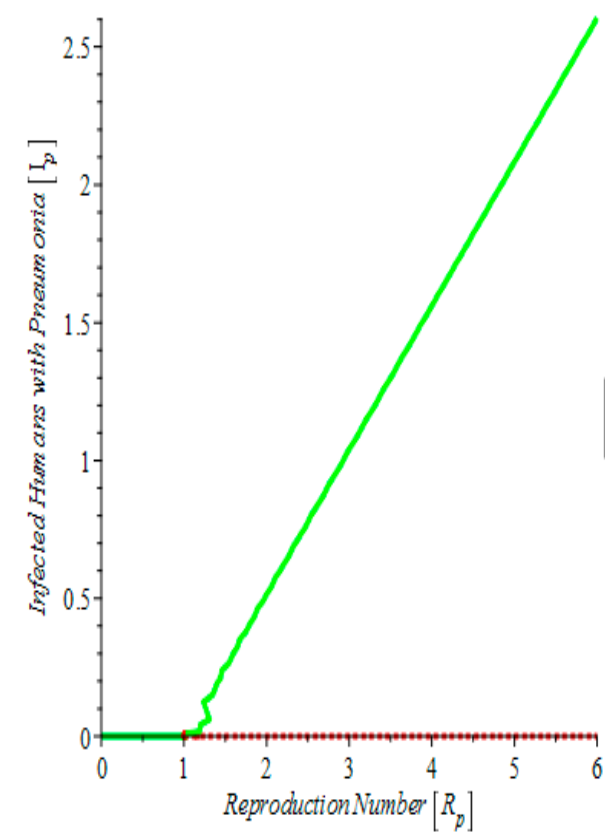

Figure 7: The forward bifurcation diagram for pneumonia infection

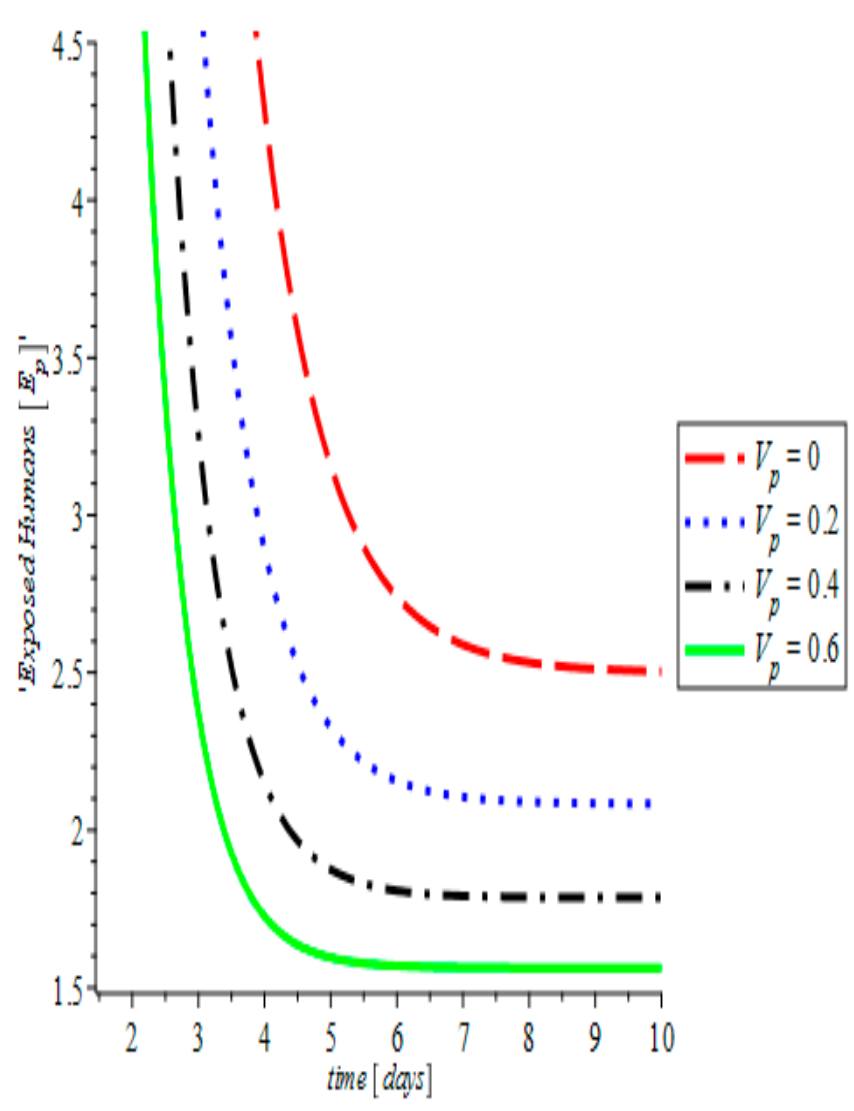

Figure 6: The variation of proportion of exposed human population to pneumonia for different values of vaccination rates $\left(V_{p}\right)$

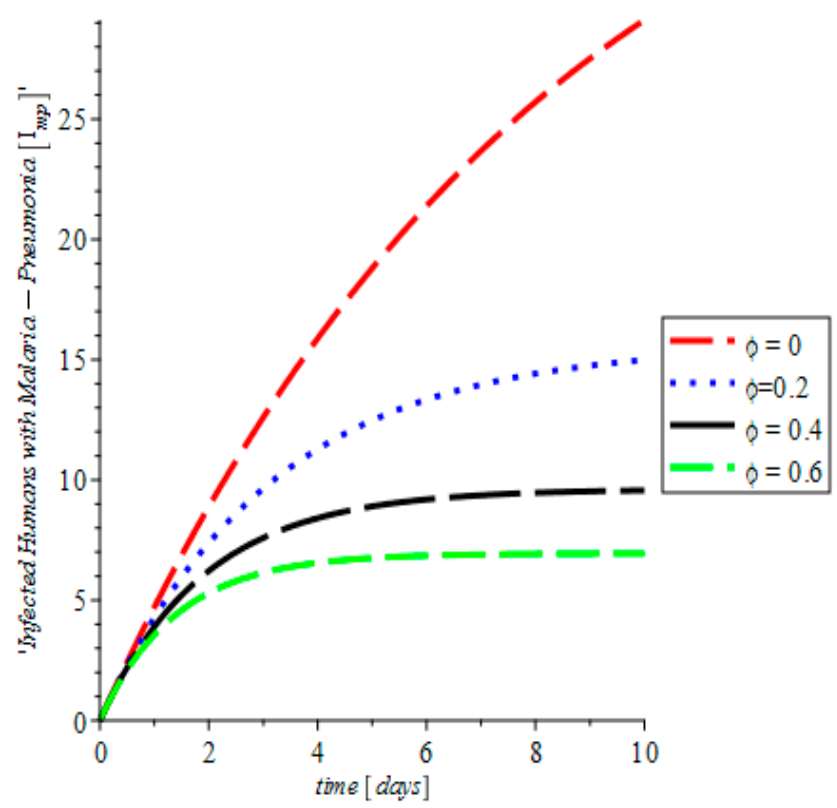

Figure 8: The variation of proportion of malariapneumonia infected human population for different values of $\phi$ 


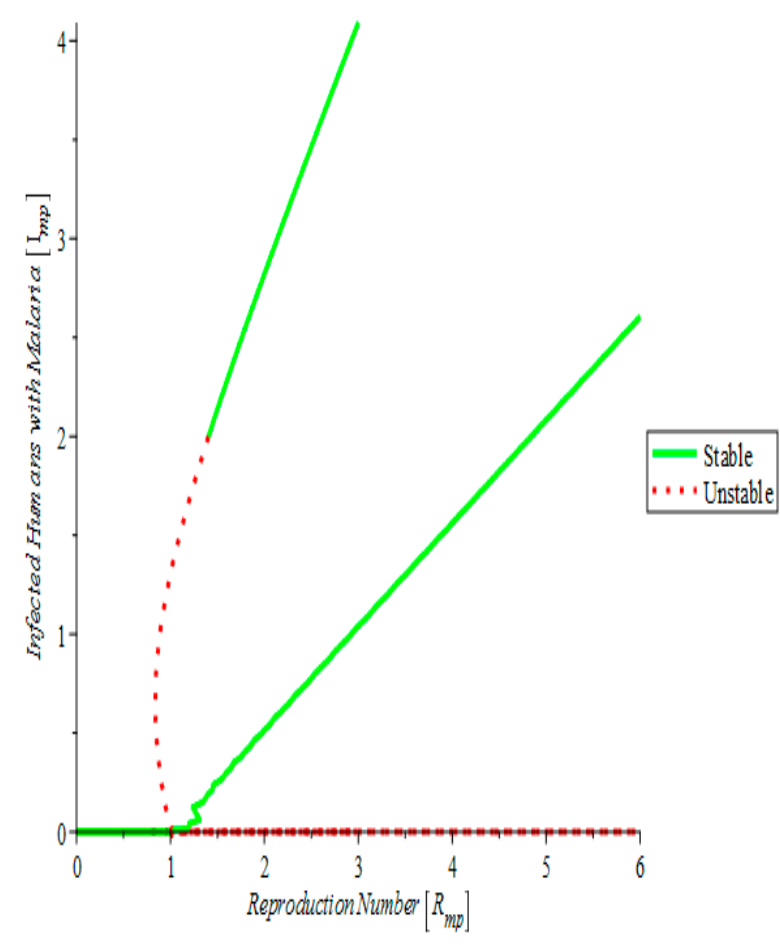

Figure 9: The co-infection bifurcation diagram for malaria and pneumonia infection

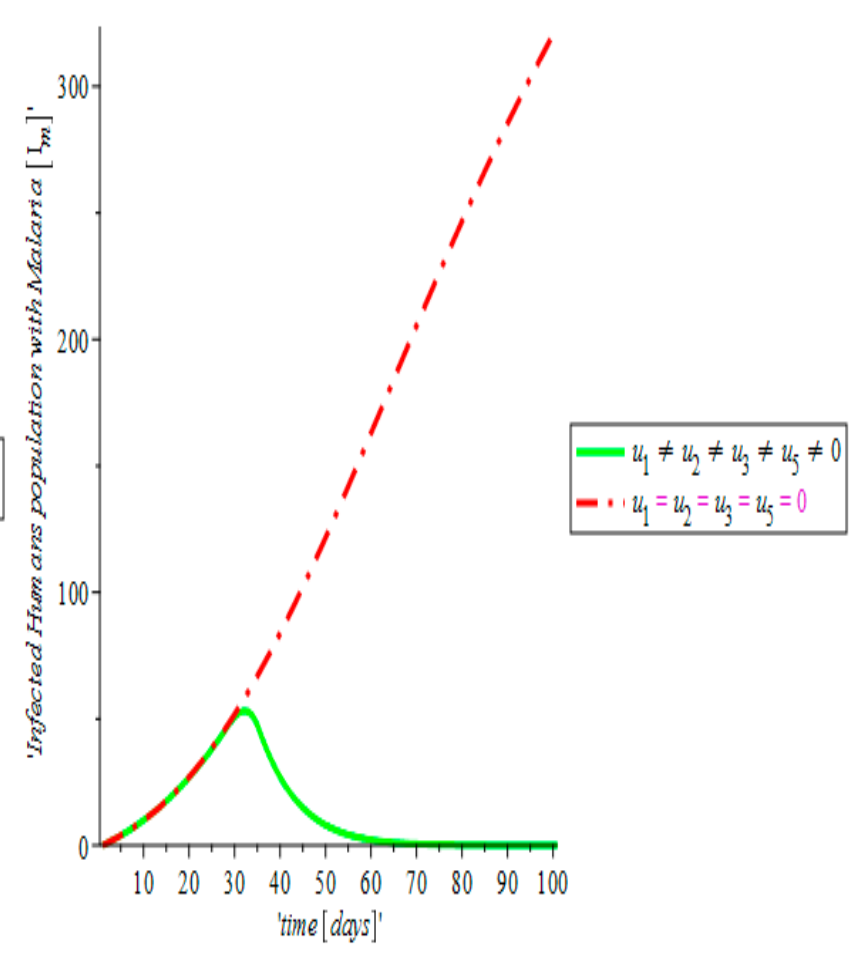

Figure 10: The variation of proportion of malaria infected population with and without control

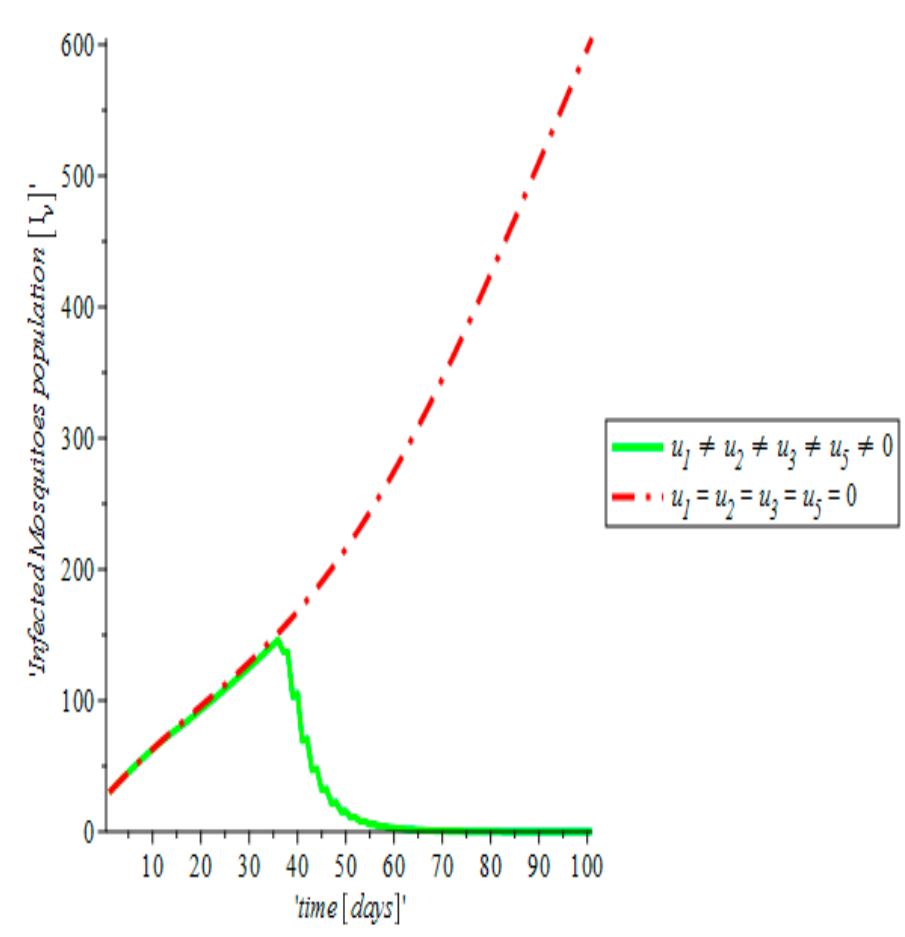

Figure 11: The variation of proportion of mosquito infected population with and without control

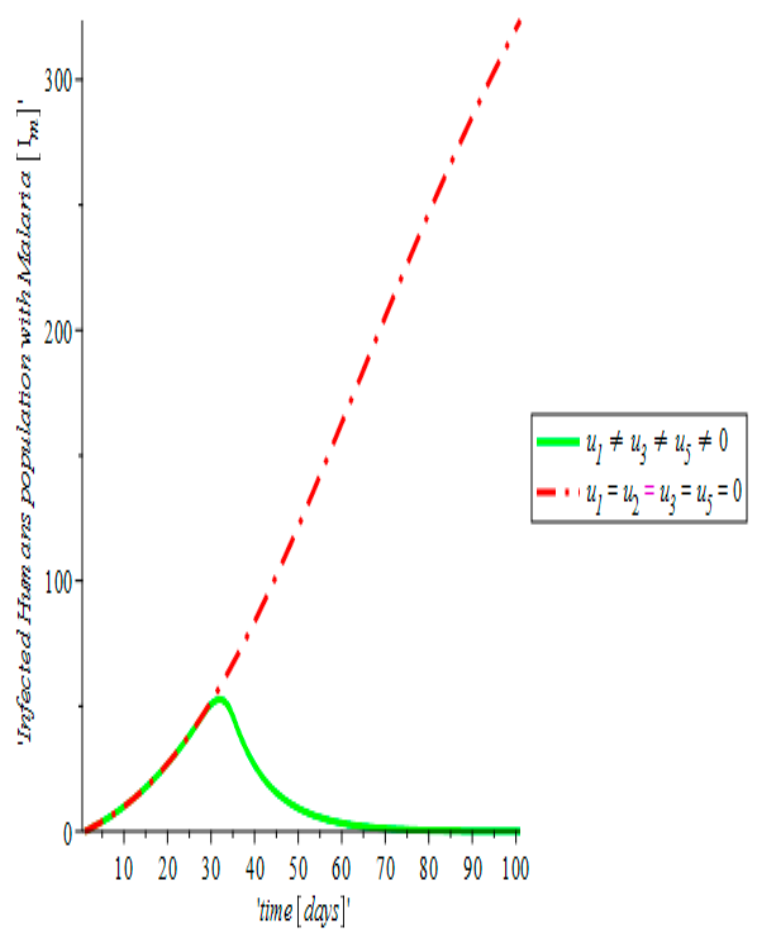

Figure 12: The variation of proportion of malaria infected population with and without control 


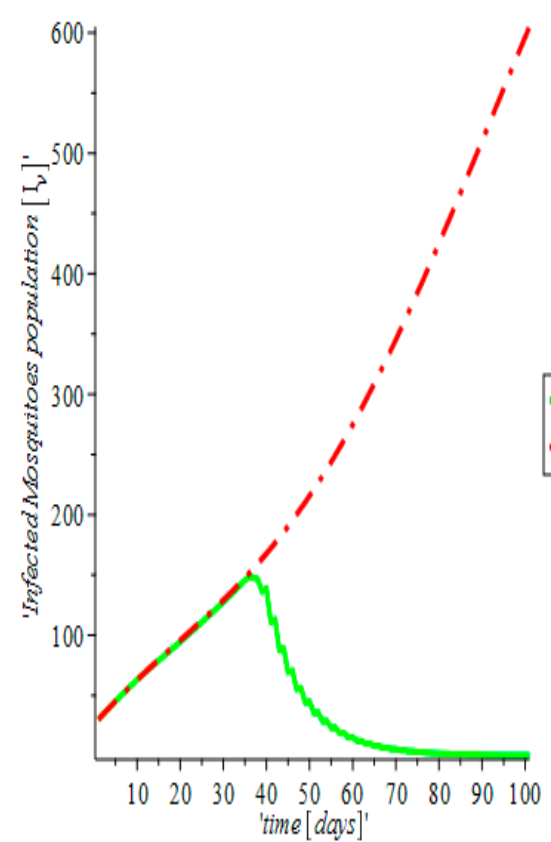

Figure 13: The variation of proportion of mosquito infected population with and without control

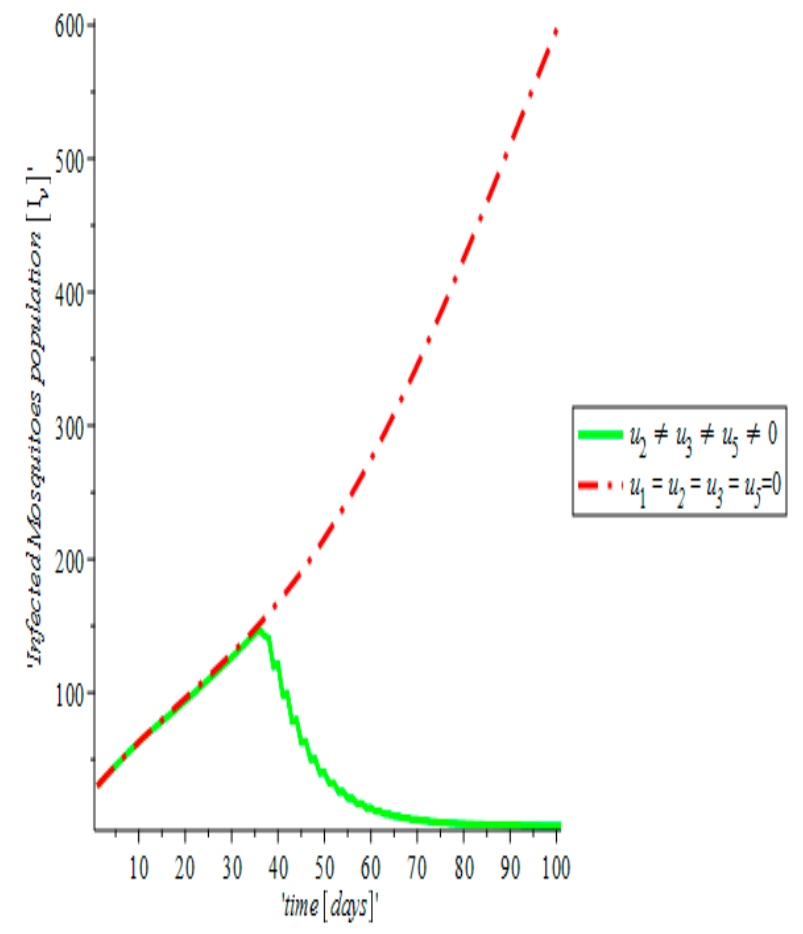

Figure 15: The variation of proportion of mosquito infected population with and without control

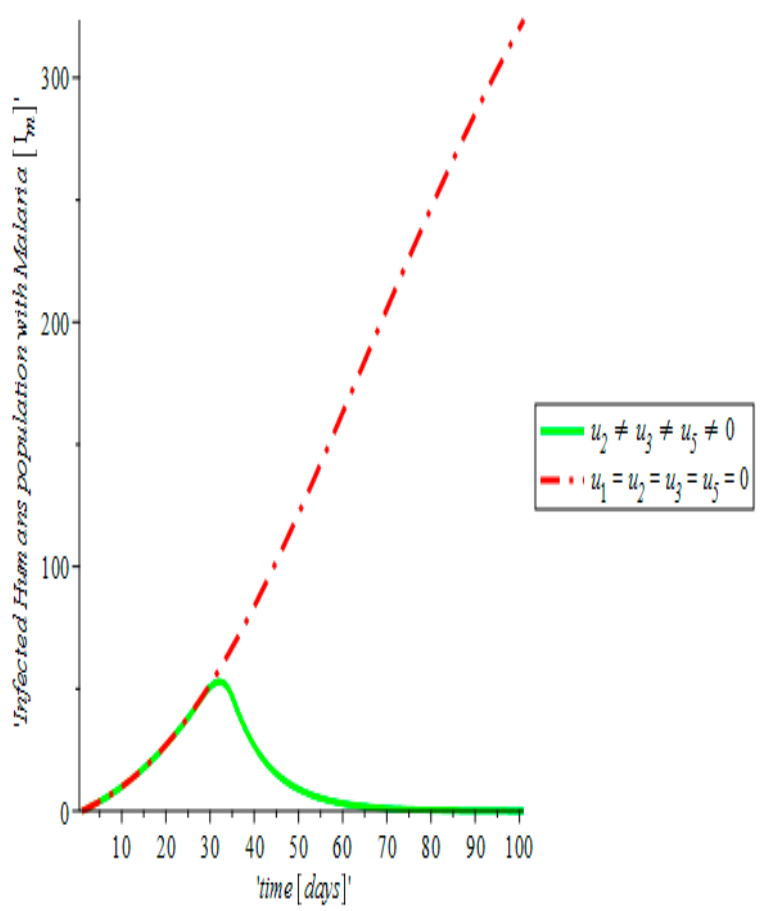

Figure 14: The variation of proportion of malaria infected population with and without control

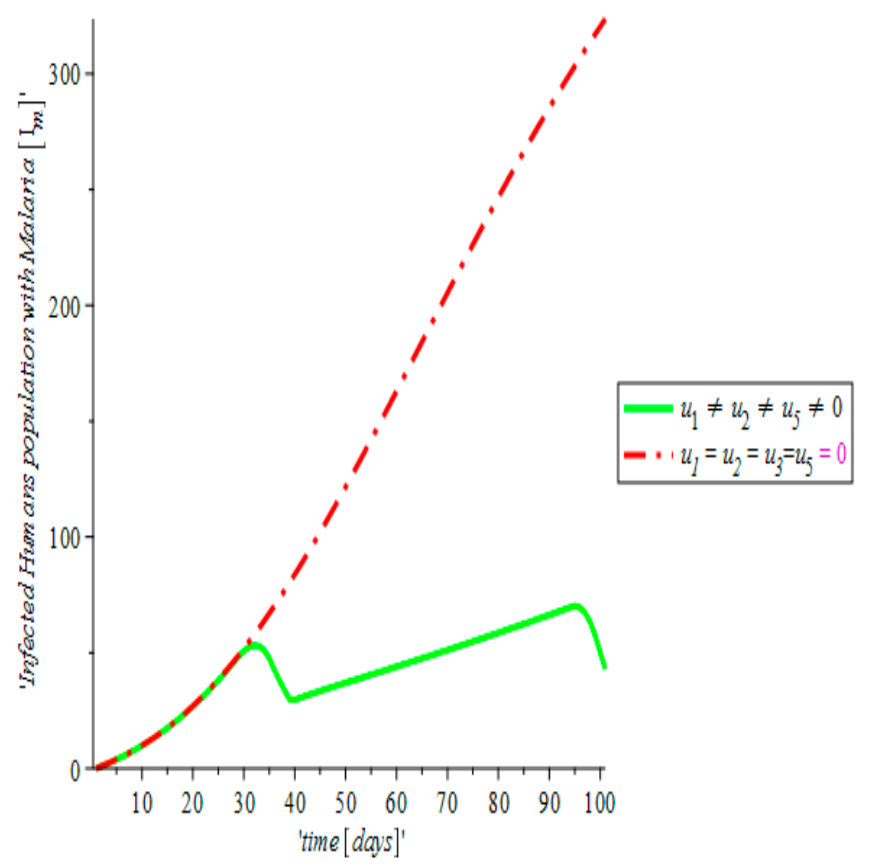

Figure 16: The variation of proportion of malaria infected population with and without control 


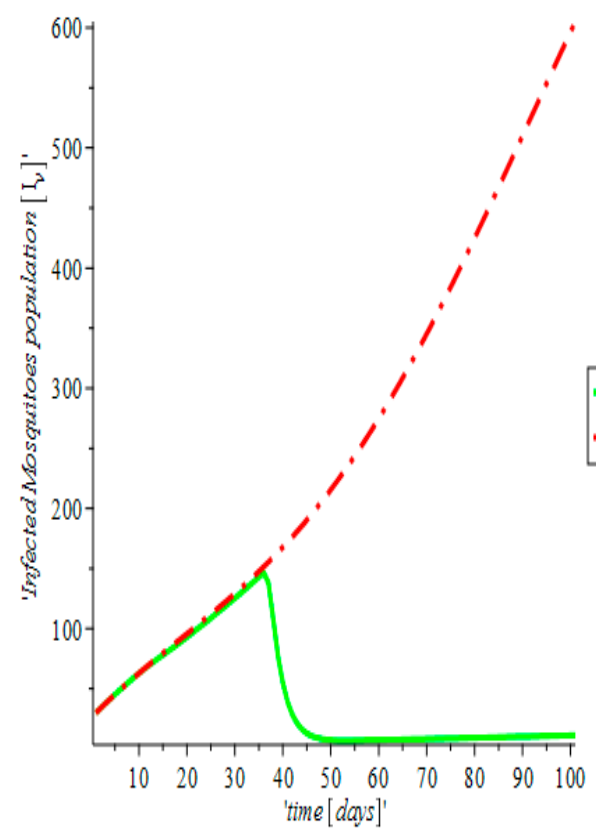

Figure 17: The variation of proportion of mosquitoes infected population with and without control

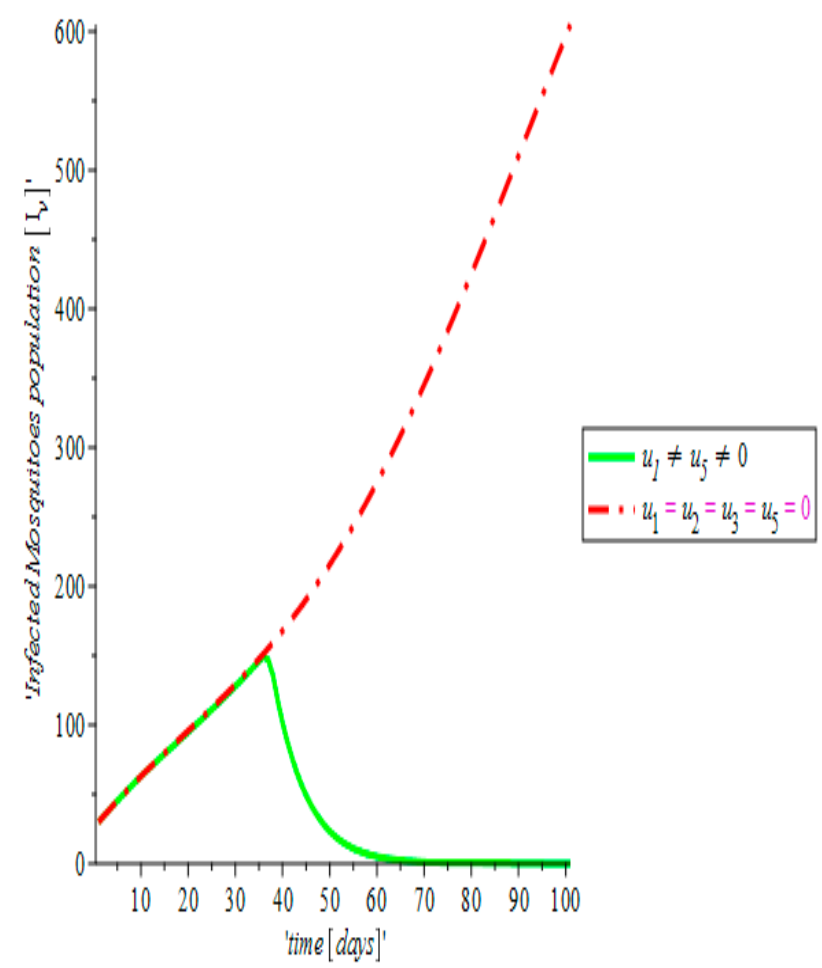

Figure 19: The variation of proportion of mosquito infected population with and without control

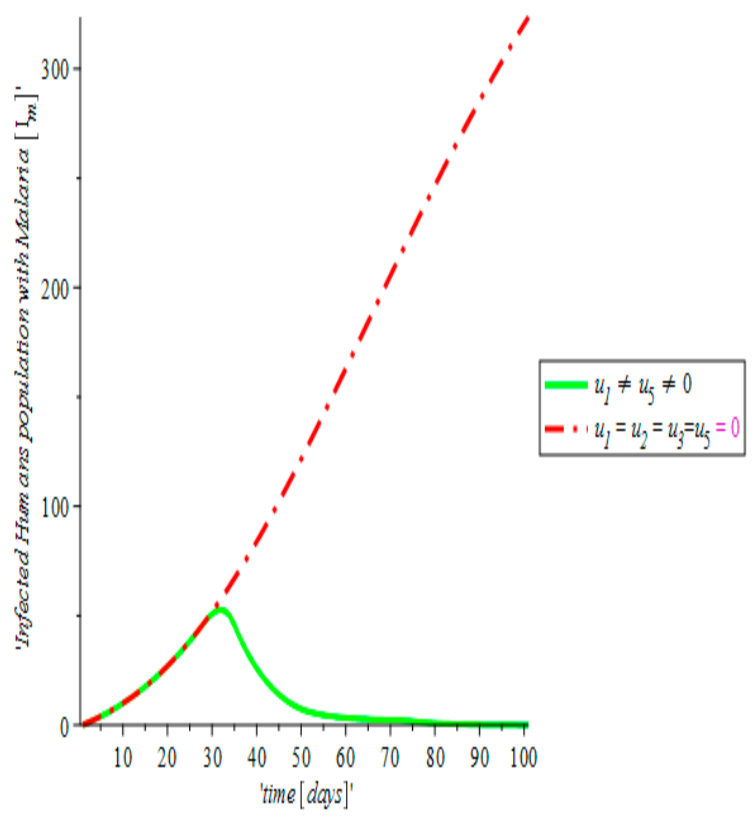

Figure 18: The variation of proportion of malaria infected population with and without control

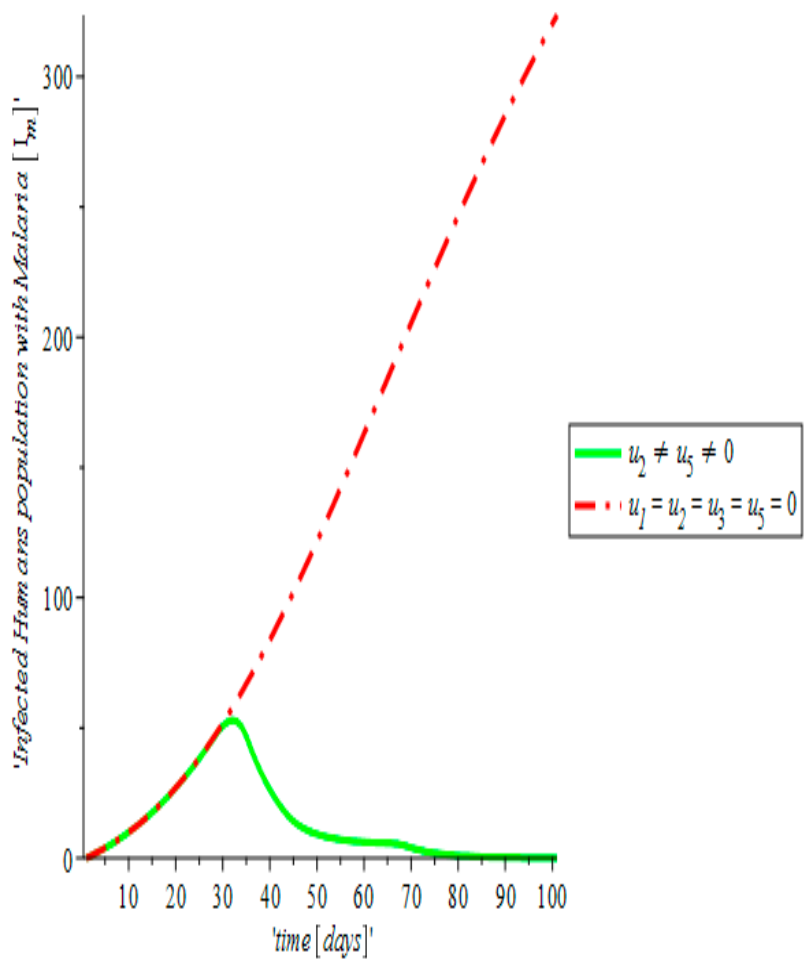

Figure 20: The variation of proportion of malaria infected population with and without control 

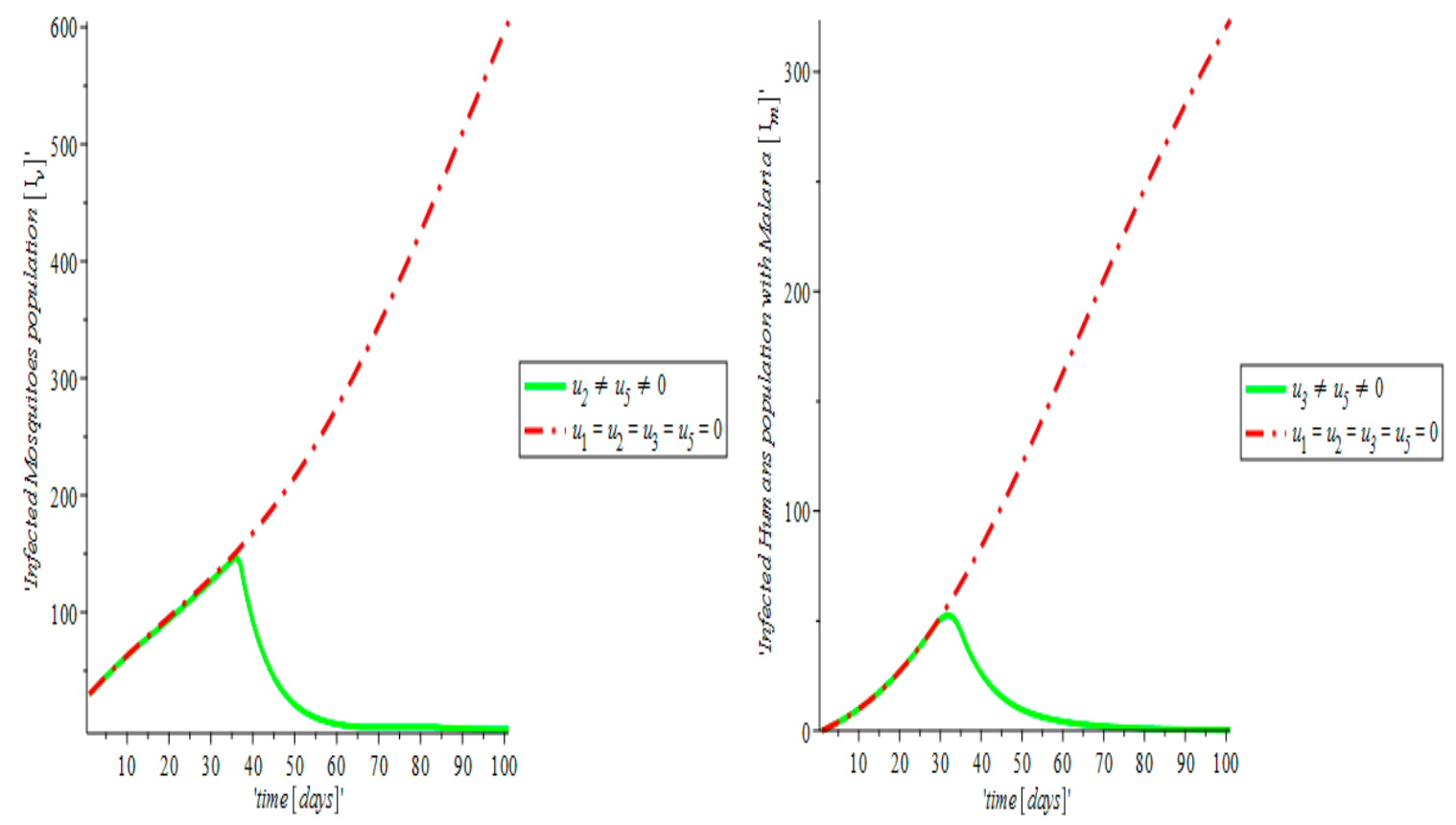

Figure 4.21: The variation of proportion Of mosquito infected population with and without control

Figure 4.22: The variation of proportion of malaria infected population with and without control
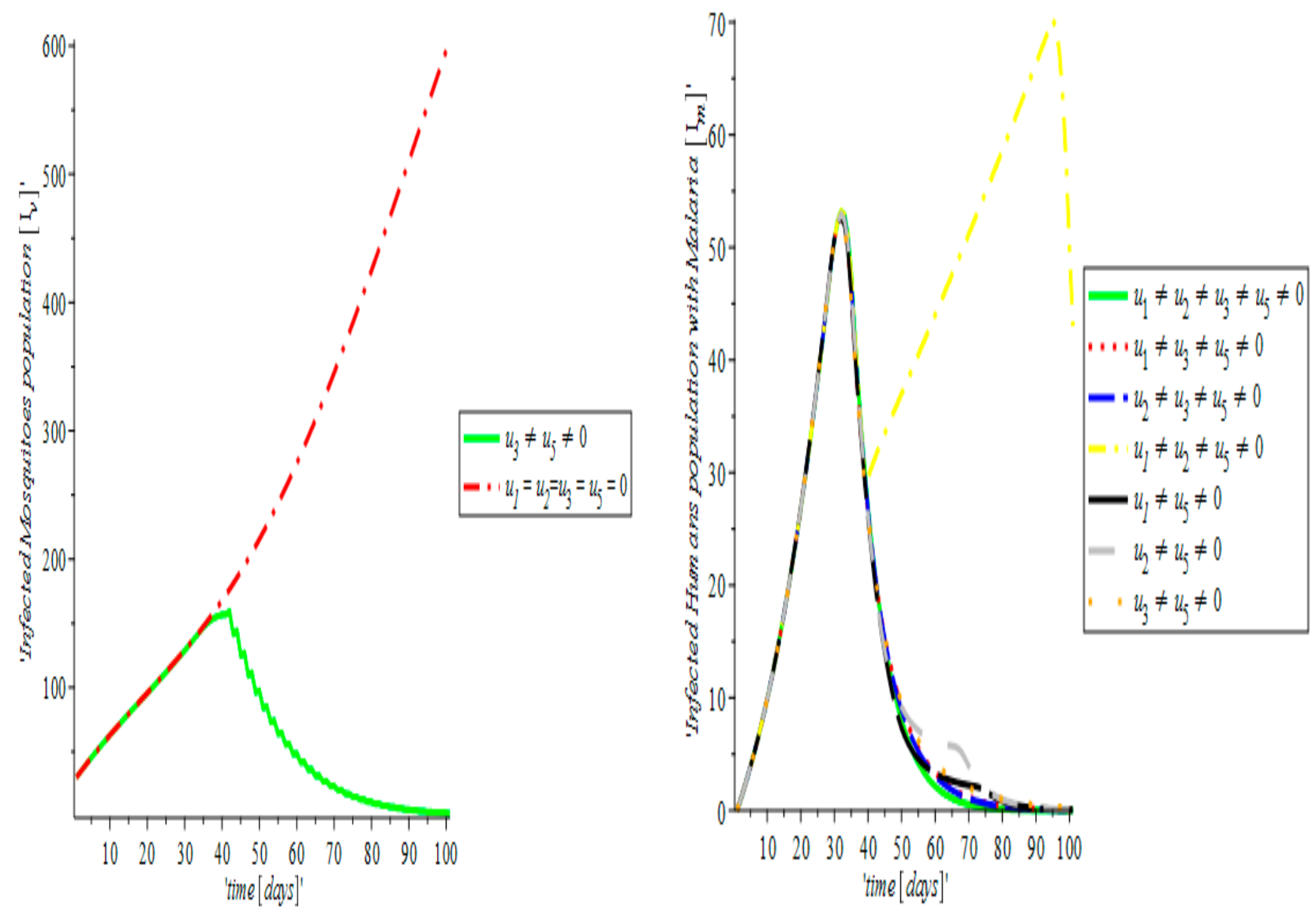

102030405060708090100 time $[\text { davs }]^{\prime}$

Figure 23: The variation of proportion of mosquito infected population with and without control

Figure 24: The comparison of the effect of the different control strategies on infected humans with malaria for the malaria model 


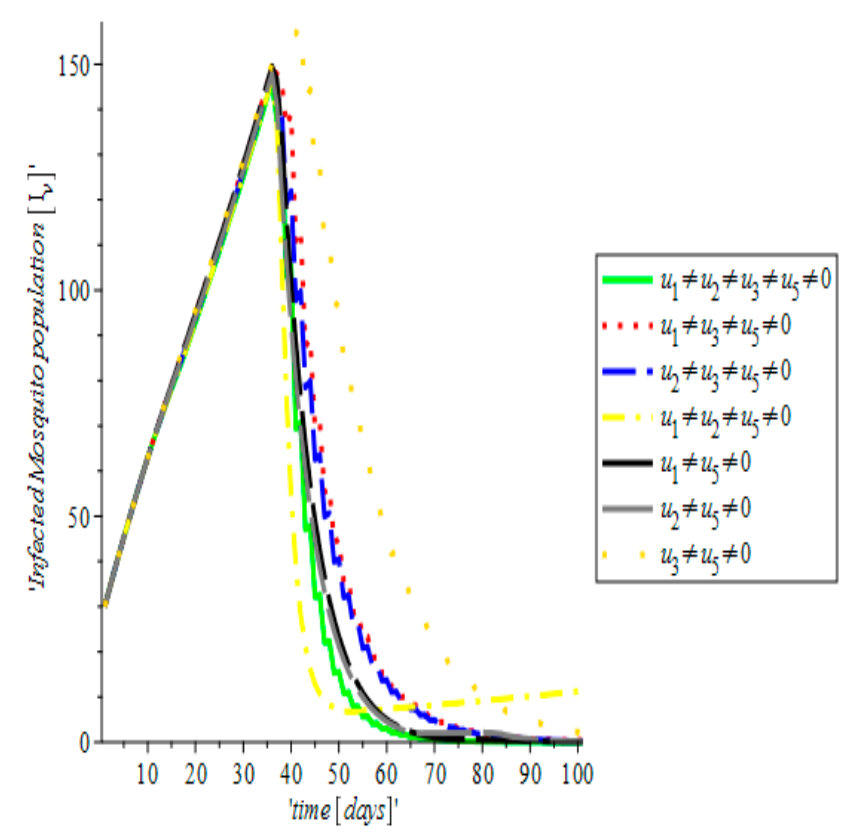

Figure 25: The comparison of the effect Of the different control strategies on infected mosquitoes for the malaria model

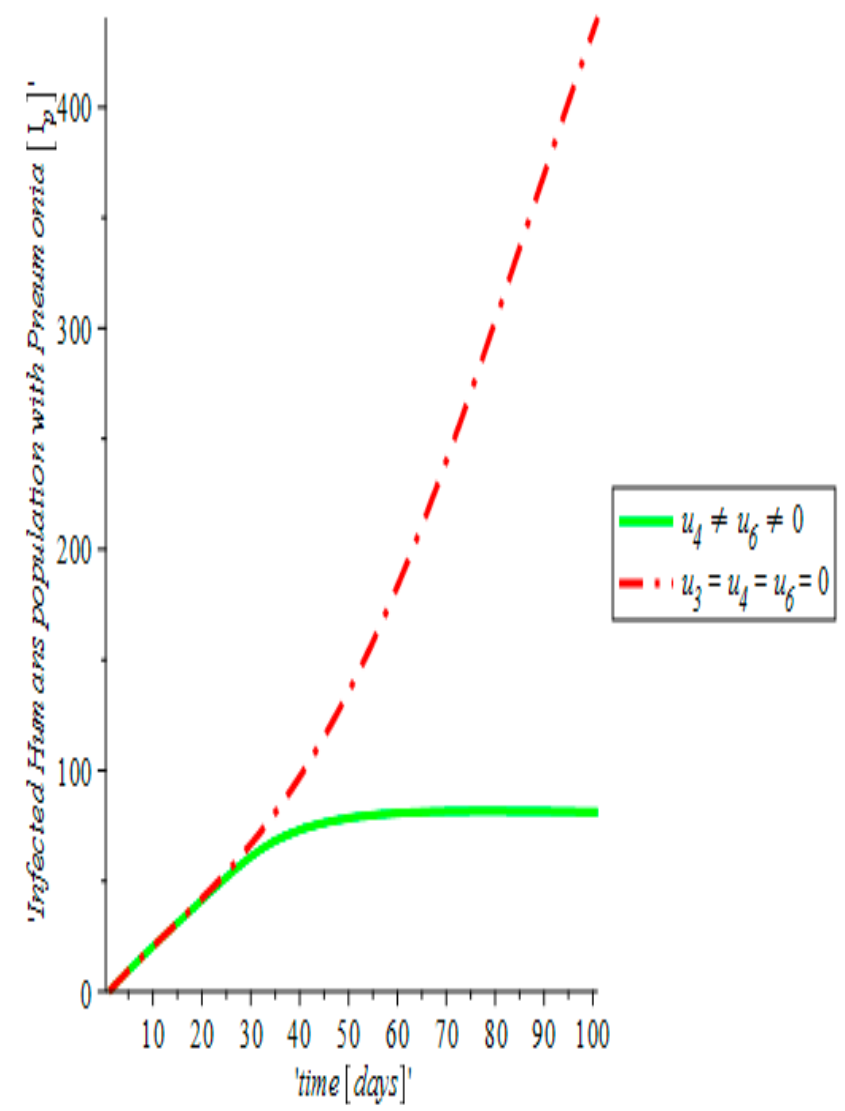

Figure 27: The variation of proportion of pneumonia infected population with and without control

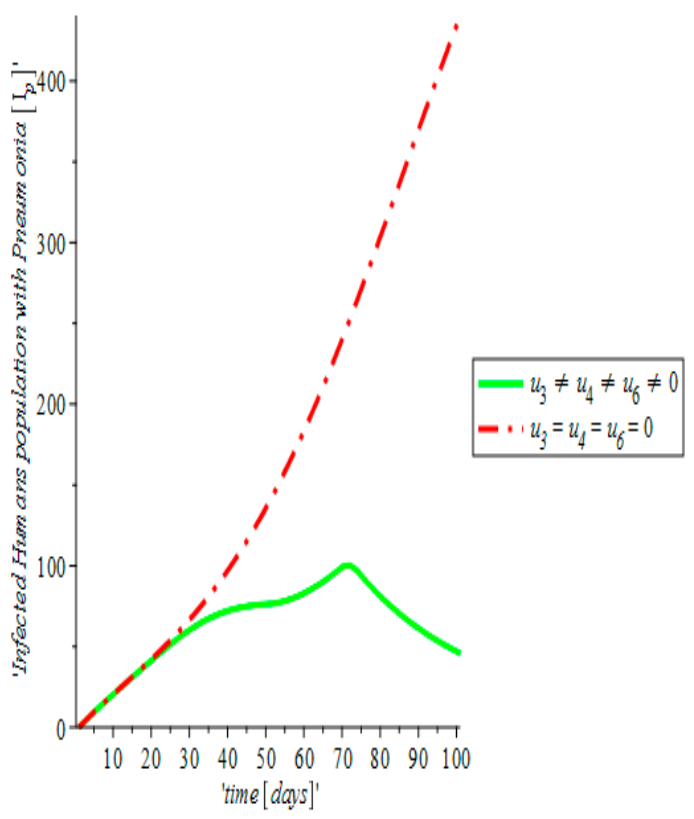

Figure 4.26: The variation of proportion of pneumonia infected population with and without control

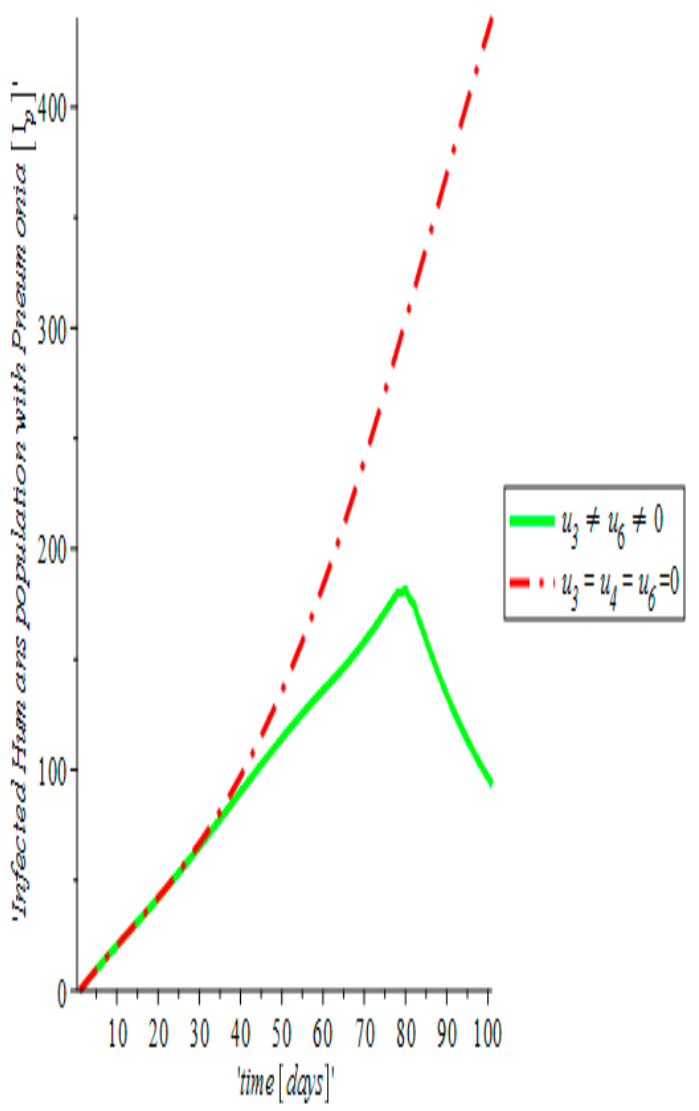

Figure 28: The variation of proportion of pneumonia infected population with and without control 


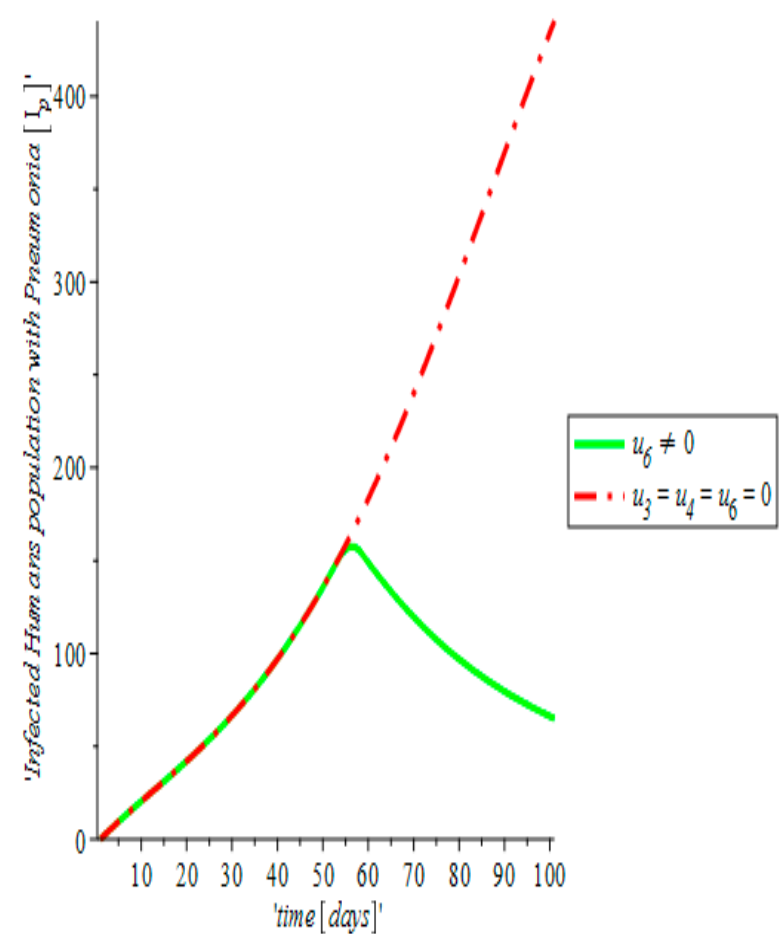

Figure 29: The variation of proportion of pneumonia infected population with and without control

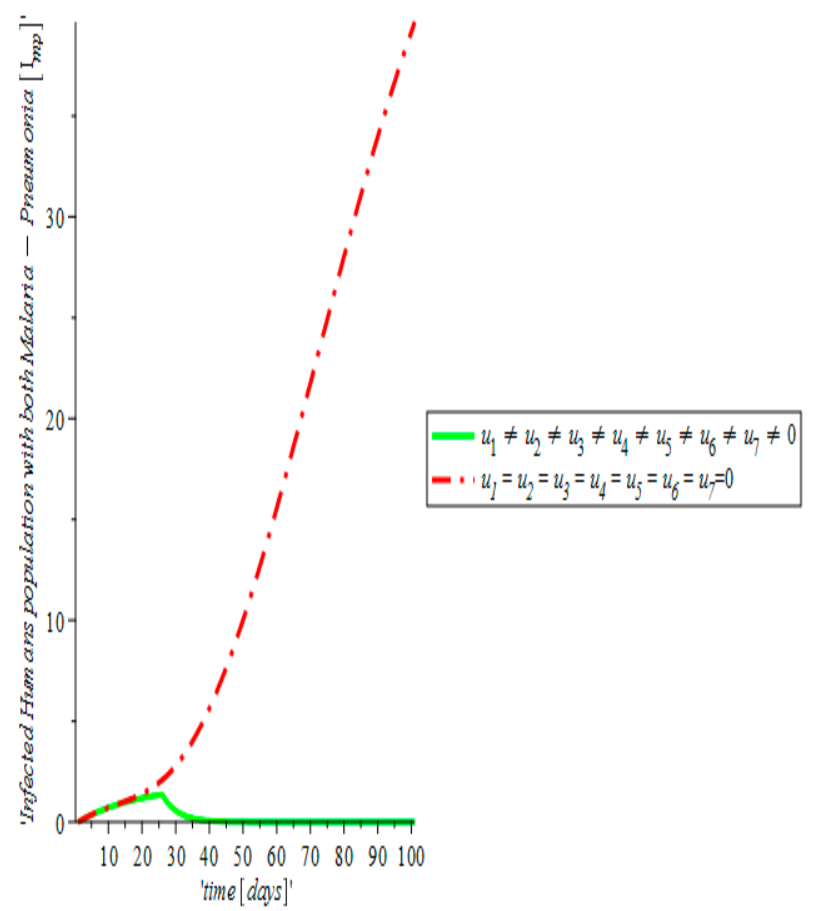

Figure 31: The variation of proportion of malaria-pneumonia infected population with and without control

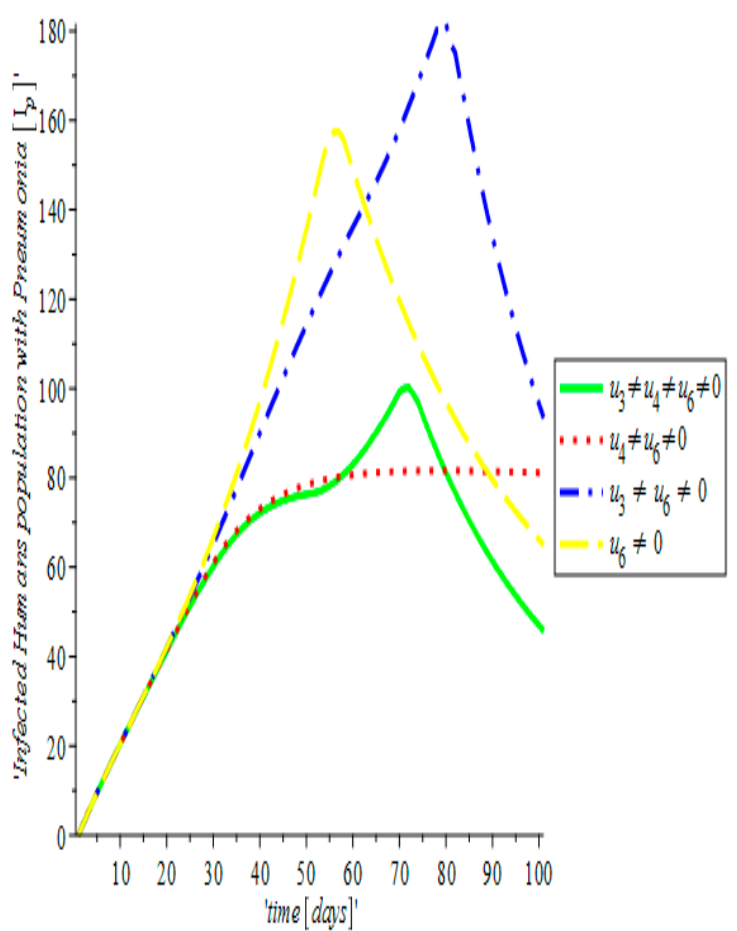

Figure 30: The comparison of the effect of the different control strategies on infected humans with pneumonia for the pneumonia model

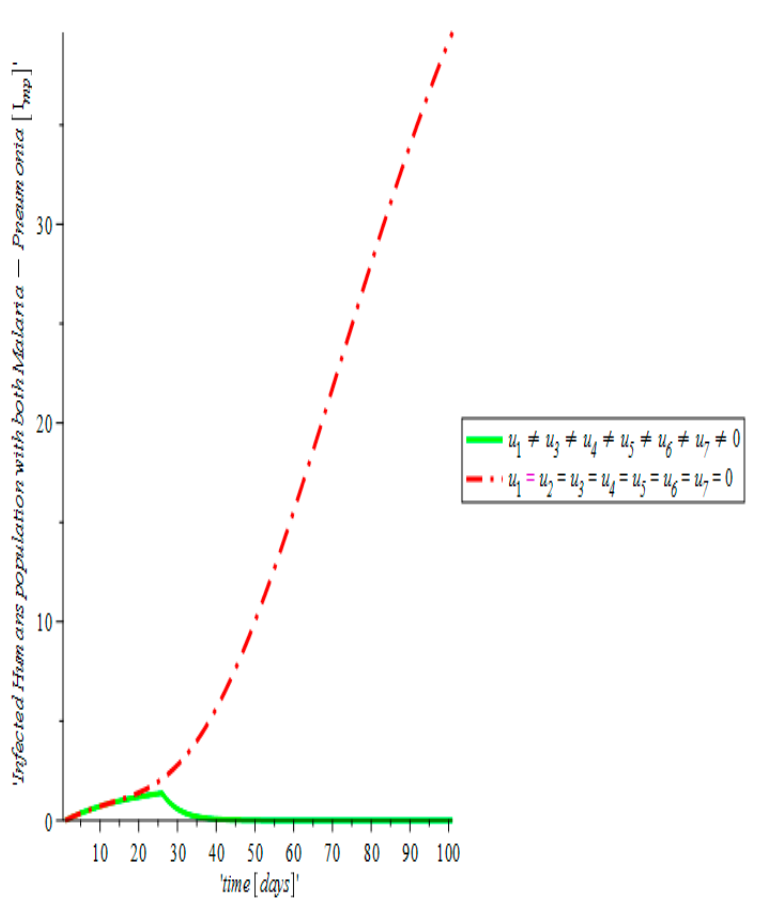

Figure 32: The variation of proportion of malaria-pneumonia infected population with and without control 


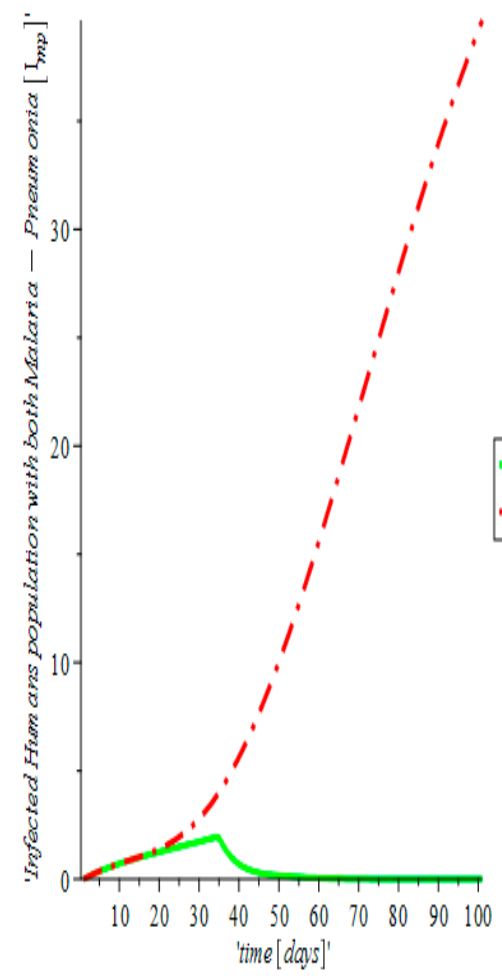

Figure 33: The variation of proportion of malaria-pneumonia infected population with and without control

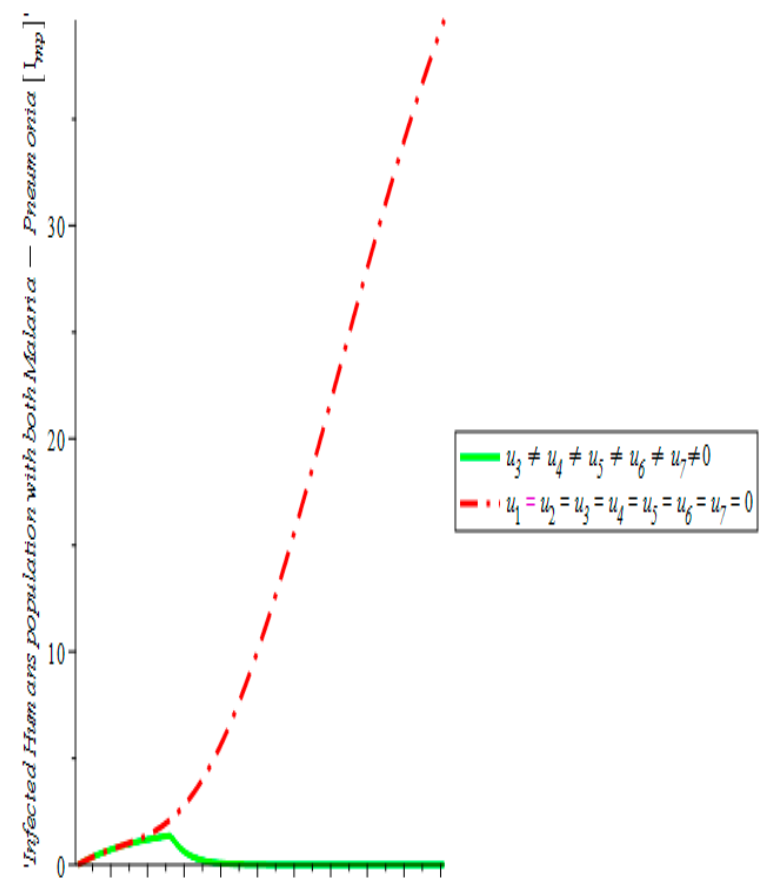

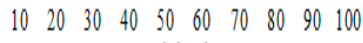
'time [doys]'

Figure 35: The variation of Proportion of malaria-pneumonia infected population with and without control

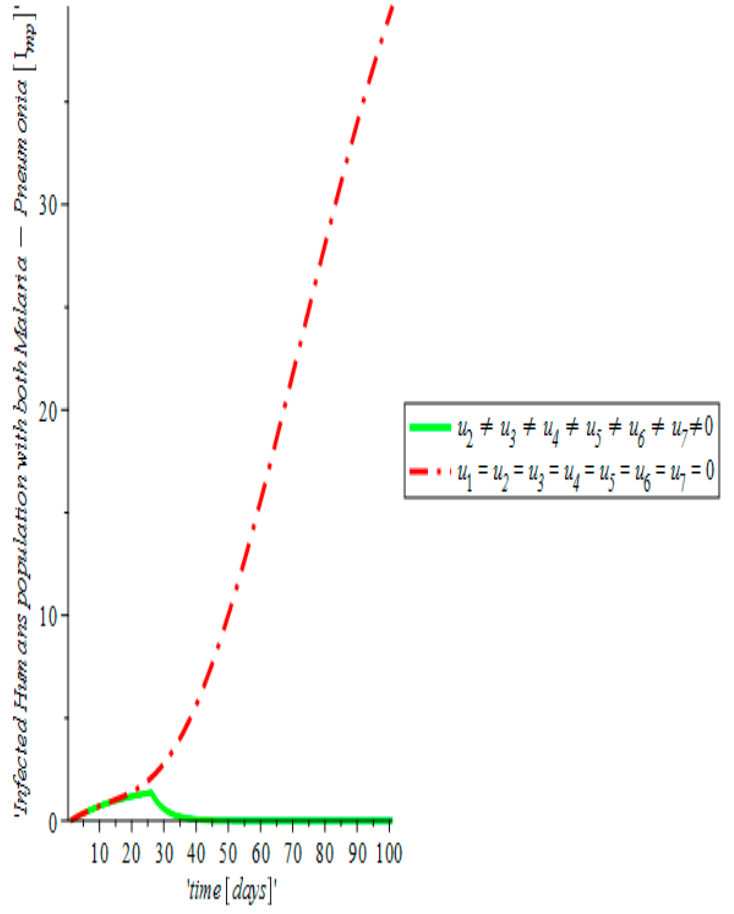

Figure 34: The variation of proportion of malaria -pneumonia infected population with and without contro

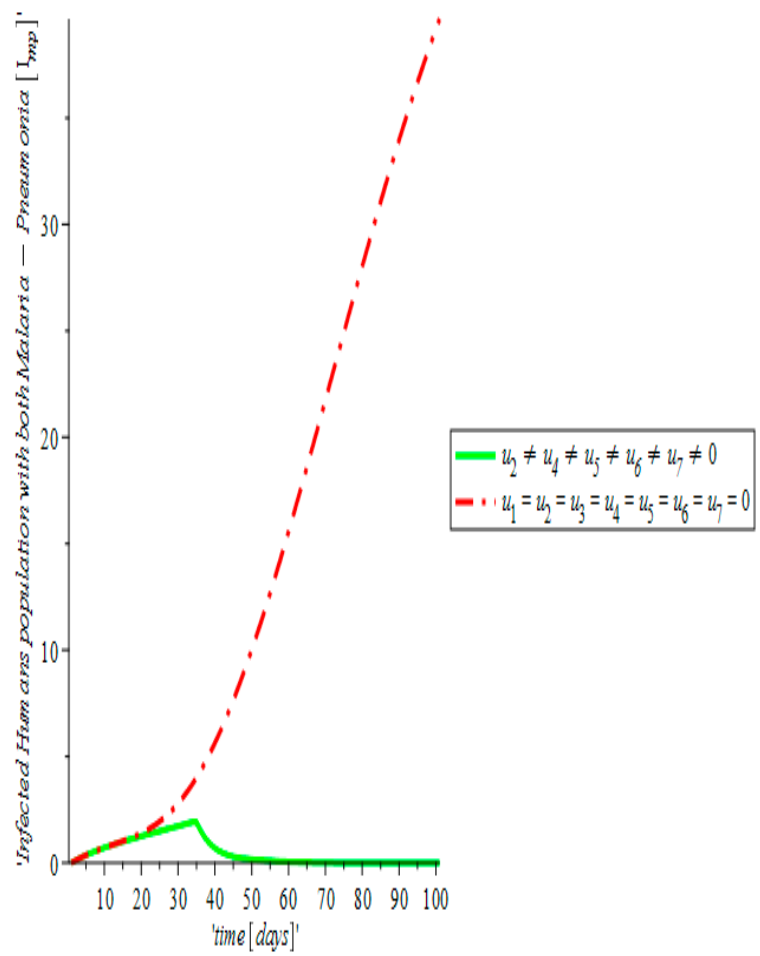

Figure 36: The variation of proportion of malariapneumonia infected population with and without control 


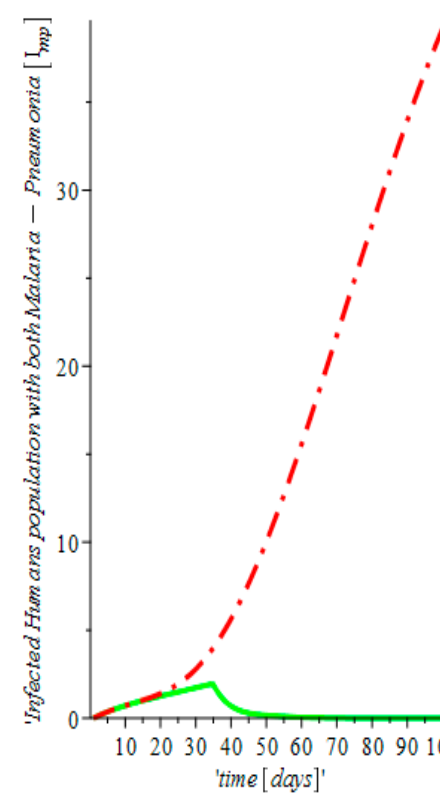

Figure 37: The variation of proportion of malaria-pneumonia infected population with and without control

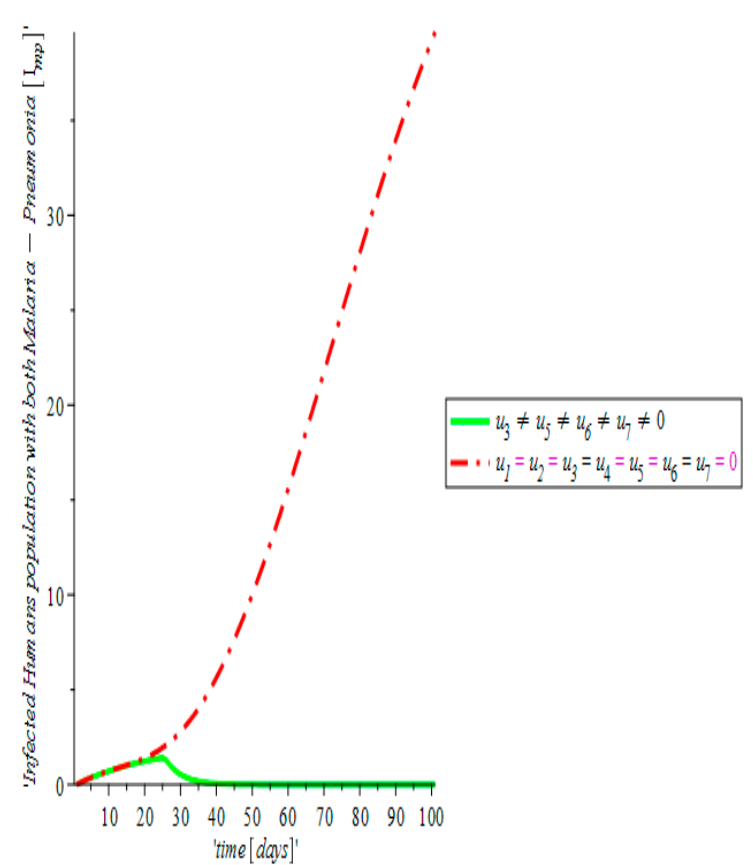

Figure 39: The variation of proportion of malaria-pneumonia infected population with and without control

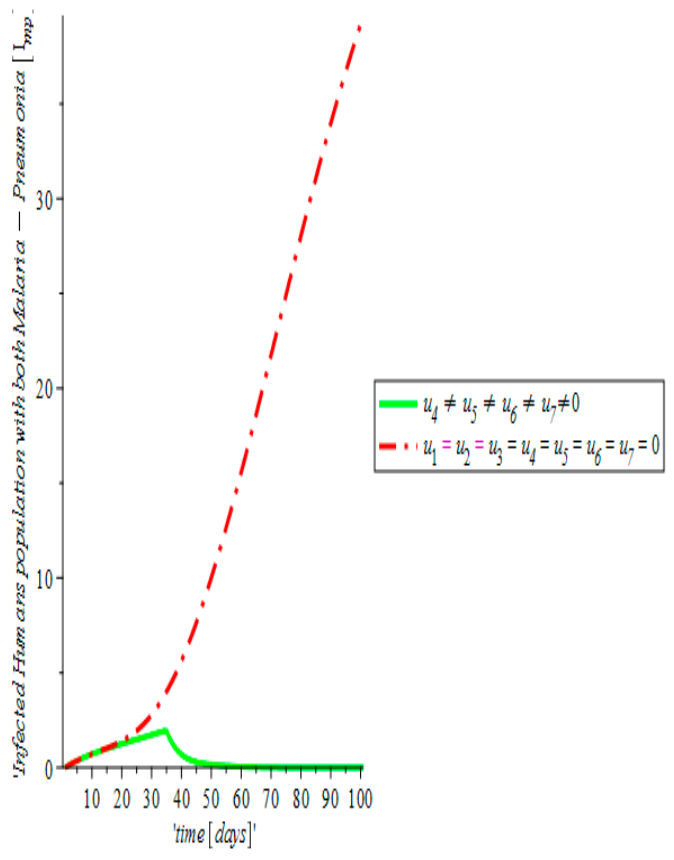

Figure 38: The variation of proportion of malaria-pneumonia infected population with and without control

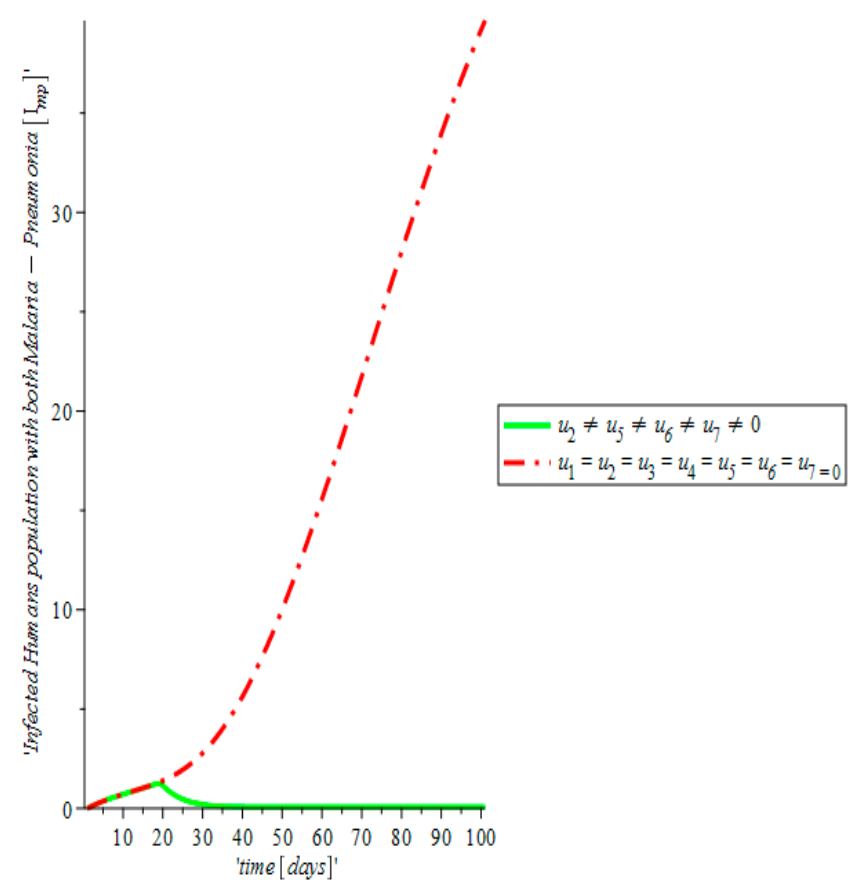

Figure 40: The variation of proportion of malariapneumonia infected population with and without control 

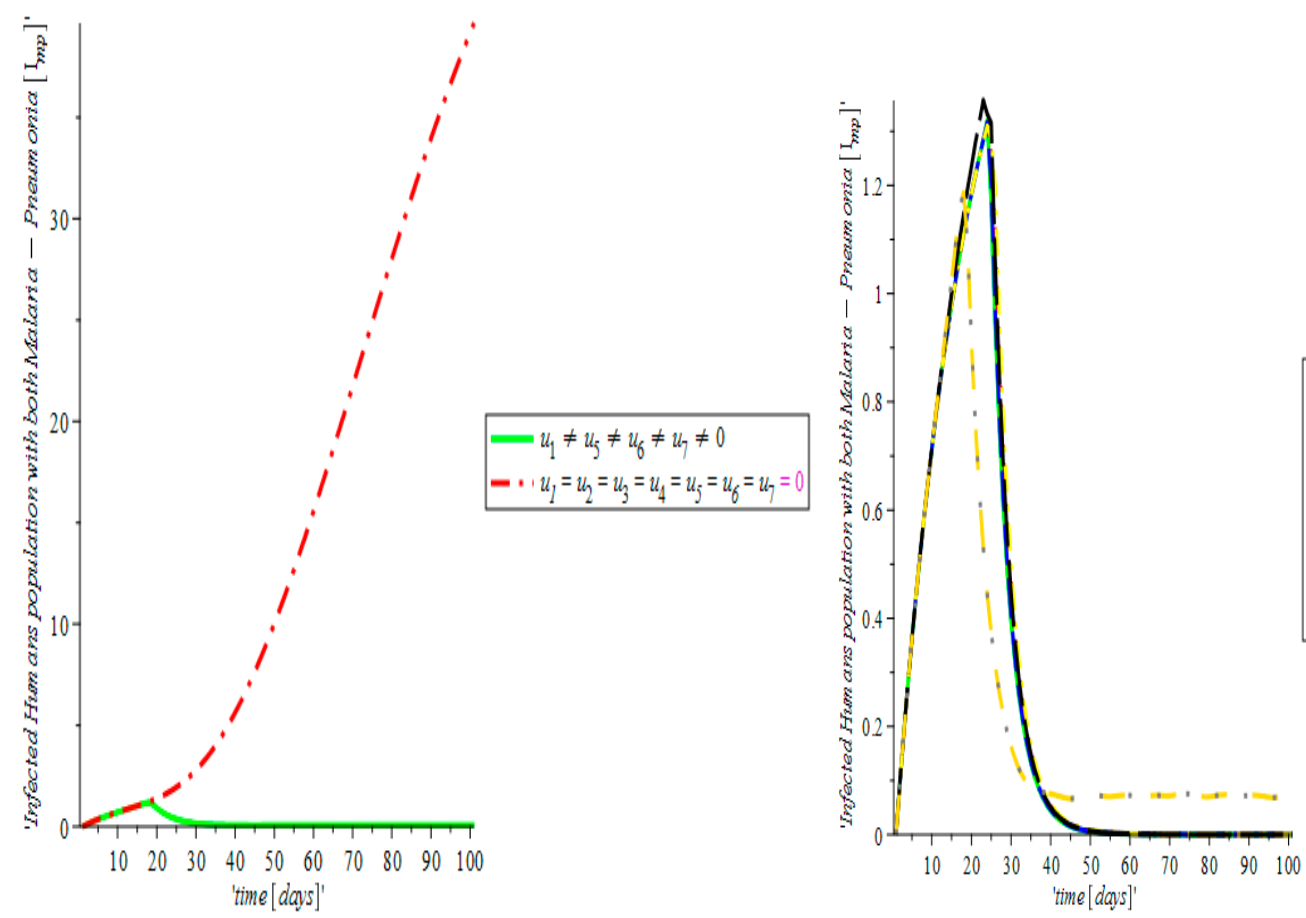

Figure 4.41: The variation of proportion of malaria-pneumonia infected population with and without control

Figure 4.42: The comparison of the effect of the different control strategies on infected humans with malaria-pneumonia for the malariapneumonia model

We further explore an optimal control model with time preventive (sleeping under treated mosquito nets $u_{1}(t)$, Insecticide spray $u_{2}(t)$ and sanitation $u_{3}(t)$ ) and treatment (use of antimalaria drug $\left.u_{5}(t)\right)$ strategies as control measures on the transmission of malaria disease. Various combinations of the controls are considered as follows:

(a) Optimal control using: sleeping under treated mosquito nets $u_{1}(t)$, insecticide spray $u_{2}(t)$, sanitation $u_{3}(t)$ and treatment $u_{5}(t)$.

Here, all the four controls are used to optimize the objective function $J$. It was observed in Figure 10 and Figure 11 that the combination of the four controls resulted in significant decrease in both the number of infected humans with malaria $\left(I_{m}\right)$ and infected mosquitoes $\left(I_{v}\right)$ (represented by the green solid line) as against the increased number of infected humans $\left(I_{m}\right)$ and infected mosquitoes $\left(I_{v}\right)$ (denoted by the red dash dot line) in the uncontrolled case.

(b) Optimal control using: sleeping under treated mosquito nets $u_{1}(t)$, sanitation $u_{3}(t)$ and treatment $u_{5}(t)$.

In this case, the control on spraying insecticide $u_{2}(t)$ is set to zero while the controls on sleeping under treated mosquito nets $u_{1}(t)$, good sanitation $u_{3}(t)$ and treatment $u_{5}(t)$ are used to optimize the objective function $J$. Using this strategy, it was observed in Figure 12 and Figure 13 that the number of infected humans with malaria $\left(I_{m}\right)$ and infected mosquitoes $\left(I_{v}\right)$ decreases as against the increase observed in the uncontrolled case.

(c) Optimal control using: insecticide spray $u_{2}(t)$, sanitation $u_{3}(t)$ and treatment $u_{5}(t)$. Here, the controls on spraying insecticide $u_{2}(t)$, good sanitation $u_{3}(t)$ and treatment $u_{5}(t)$ are used to optimize the objective function $J$ while the control on sleeping under mosquito treated nets $u_{1}(t)$ is set to zero. Considering this strategy, it was observed in Figure 14 and Figure 15 that the number of infected humans with malaria $\left(I_{m}\right)$ and infected mosquitoes $\left(I_{v}\right)$ decreases as against the increase observed in the uncontrolled case respectively. 
(d) Optimal control using: sleeping under treated mosquito nets $u_{1}(t)$, insecticide spray $u_{2}(t)$ and treatment $u_{5}(t)$.

The control on sleeping under mosquito treated nets $u_{1}(t)$, insecticide spray $u_{2}(t)$ and treatment $u_{5}(t)$ are used to optimize the objective function $J$ while the control on sanitation was set to zero. It was observed in Figure 16 that the control measure employed here decreases the number of infected humans with malaria $\left(I_{m}\right)$ but not to the level of the controls involving good sanitation as against the increase observed in the uncontrolled case. Although, a decrease in the number of infected mosquitoes $\left(I_{v}\right)$ was observed as against the uncontrolled case in Figure $\mathbf{1 7}$ but due to poor or no sanitation, the number of infected mosquitoes $\left(I_{v}\right)$ began to rise.

(e) Optimal control using: sleeping under treated mosquito nets $u_{1}(t)$ and treatment $u_{5}(t)$.

With this strategy, the controls sleeping under treated mosquito nets $u_{1}(t)$ and treatment $u_{5}(t)$ are used to optimize the objective function $J$ with the control on insecticide spray $u_{2}(t)$ and good sanitation $u_{3}(t)$ all set to zero. For this strategy, it was shown in Figure 18 and Figure 19 that the number of infected humans with malaria $\left(I_{m}\right)$ and infected mosquitoes $\left(I_{v}\right)$ reduces considerably from the uncontrolled case

(f) Optimal control using: insecticide spray $u_{2}(t)$ and treatment $u_{5}(t)$

With this strategy, the controls insecticide spray $u_{2}(t)$ and treatment $u_{5}(t)$ are used to optimize the objective function $J$, while the control on sleeping under mosquito treated nets $u_{1}(t)$ and good sanitation $u_{3}(t)$ all set to zero. The result in Figure 20 and Figure 21 showed a significant difference in the number of infected humans with malaria $\left(I_{m}\right)$ and infected mosquitoes $\left(I_{v}\right)$ with optimal strategy compared to $\left(I_{m}\right)$ and $\left(I_{v}\right)$ without control. It was observed in Figure 20 that the control strategies resulted in a decrease in the number of infected humans with malaria (solid green line) as against an increase in the uncontrolled case (red dash dot line). Also, in Figure 21, the uncontrolled case resulted in increased number of infected mosquitoes $\left(I_{v}\right)$, while the control strategy lead to a drastic decrease in the number of infected mosquitoes $\left(I_{v}\right)$.

(g) Optimal control using: sanitation $u_{3}(t)$ and treatment $u_{5}(t)$.

The objective function $J$ are optimized in this case using the control strategy on good sanitation $u_{3}(t)$ and treatment $u_{5}(t)$, while the control on sleeping treated mosquito nets $u_{1}(t)$ and insecticide spray $u_{2}(t)$ are set to zero. It was observed in Figure 22 that the control strategies resulted in a decrease in the number of infected humans $\left(I_{m}\right)$ with malaria as against increase in the uncontrolled case. Similarly, in Figure 23, the uncontrolled case resulted in increased number of infected mosquitoes $\left(I_{v}\right)$, while the control strategy lead to a decrease in the number of infected mosquitoes.

(h) Comparing all the control strategies

A comparison of all the control strategies for our malaria model case 4 was considered on both infected humans $\left(I_{m}\right)$ and mosquitoes $\left(I_{v}\right)$ in Figure 24 and Figure 25 respectively. It was observed that all the four controls led to a decrease in the number of infected humans $\left(I_{m}\right)$ and mosquitoes $\left(I_{v}\right)$ respectively. The control strategies $\left(u_{1}(t), u_{3}(t), u_{5}(t)\right)$ and $\left(u_{2}(t), u_{3}(t), u_{5}(t)\right)$ also led to a decrease in the number of infected humans and mosquitoes respectively. The strategy $\left(u_{1}(t), u_{2}(t), u_{5}(t)\right)$ yield a poorer result on both the infected humans and mosquitoes. This result showed that with individuals sleeping under mosquito treated nets, spraying of insecticide in the sleeping area and have access to treatment may not be sufficient to control the spread of the disease in the community if poor sanitation is encouraged. The optimal strategies involving two controls namely: $\left(u_{1}(t), u_{5}(t)\right),\left(u_{2}(t), u_{5}(t)\right)$ and $\left(u_{3}(t), u_{5}(t)\right)$ decreases the number of infected humans $\left(I_{m}\right)$ and mosquitoes $\left(I_{v}\right)$. From Figure 24 and Figure 25, it was also observed that the optimal control strategies involving good sanitation gave a better result in terms of reducing the number of infected humans $\left(I_{m}\right)$ and mosquitoes $\left(I_{v}\right)$. 
The result of the optimal control for pneumonia is discussed using time dependent preventive and treatment controls to investigate the effect of these controls on the transmission dynamics of pneumonia disease. The following scenarios of the controls are considered:

(a) Optimal control using: sanitation $u_{3}(t)$, vaccination $u_{4}(t)$ and treatment $u_{6}(t)$ The objective function $J$ is optimized here using all the three controls sanitation $u_{3}(t)$, vaccination $u_{4}(t)$ and treatment $u_{6}(t)$. We observed from Figure 26 that the combination of the three controls resulted in significant reduction in the number of infected humans with pneumonia $\left(I_{p}\right)$ (represented by solid green line) as against the increased number of infected humans with pneumonia disease $\left(I_{p}\right)$ (denoted by red dash dot line) in the uncontrolled case.

(b) Optimal control using: vaccination $u_{4}(t)$ and treatment $u_{6}(t)$

In this case, the control on using vaccination $u_{4}(t)$ and treatment $u_{6}(t)$ are used to optimize the objective function $J$ while the control on sanitation $u_{3}(t)$ is set to zero. For this strategy, it was observed in Figure 27 that the number of infected human with pneumonia reduce considerably and maintain a constant level over time while the number of infected humns with pneumonia increases in the uncontrolled case.

(c) Optimal control using: sanitation $u_{3}(t)$ and treatment $u_{6}(t)$

Here, the control on using sanitation $u_{3}(t)$ and treatment $u_{6}(t)$ are used to optimize the objective function $J$ with the control on vaccination $u_{4}(t)$ set to zero. The number of infected humans with pneumonia was observed to be decreasing as shown in Figure 28 while the number of infected humans with pneumonia increases considerably in the uncontrolled case.

(d) Optimal control using: treatment $\boldsymbol{u}_{6}(t)$

With this strategy, the control on using only treatment $u_{6}(t)$ was used to optimize the objective function $J$ while the controls on sanitation $u_{3}(t)$ and vaccination $u_{4}(t)$ are set to zero. The result in Figure 29 revealed that for the controlled case, the number of infected humans with pneumonia reduces (green line) while the number of infected human with pneumonia increases for the uncontrolled case (red dash dot line).

(e) Comparing all the control strategies

A comparison of all the control strategies for the pneumonia model case 5 was considered on the infected humans $\left(I_{p}\right)$ Figure 30. It was observed that all the four controls led to a decrease in the number of infected humans $\left(I_{p}\right)$ this was closely followed by the strategy of using anti-pneumonia as treatment. The strategy involving vaccination and treatment also gave a good result in reducing the number of infected humans $\left(I_{p}\right)$ and also ensure that exposed humans do not progress to the infected class. Lastly, the strategy involving the use of good sanitation and treatment also gave a good result in decreasing the number of infected humans $\left(I_{p}\right)$.

The malaria-pneumonia model with time dependent preventive and treatment control strategies is considered in this section. The following scenarios are considered as follows:

(a) Optimal control using: sleeping under mosquito treated nets $u_{1}(t)$, insecticide spray $u_{2}(t)$, sanitation $u_{3}(t)$, vaccination $u_{4}(t)$, treatment for malaria $u_{5}(t)$, treatment for pneumonia $u_{6}(t)$, treatment for both malaria and pneumonia $u_{7}(t)$

The objective function $J$ for malaria-pneumonia model is optimized in this case using all the seven controls. It was seen in Figure 31 that the combinations of the seven controls resulted in significant decrease in the number of infected humans with malaria-pneumonia $\left(I_{m p}\right)$ (solid green line) as against the increase observed in the number of infected humans $\left(I_{m p}\right)$ (dash dot red line) in the uncontrolled case.

(b) Optimal control using: sleeping under mosquito treated nets $u_{1}(t)$, sanitation $u_{3}(t)$, vaccination $u_{4}(t)$, treatment for malaria $u_{5}(t)$, treatment for pneumonia $u_{6}(t)$, treatment for both malaria and pneumonia $u_{7}(t)$ 
In this case, the control on insecticide spray $u_{2}(t)$ is set to zero while the controls on sleeping under mosquito treated nets $u_{1}(t)$, sanitation $u_{3}(t)$, vaccination $u_{4}(t)$, treatment for malaria $u_{5}(t)$, treatment for pneumonia $u_{6}(t)$, treatment for both malaria and pneumonia $u_{7}(t)$ are used to optimize the objective function $J$. Using this strategy, it was observed in Figure 32 that the number of infected humans with malaria-pneumonia $\left(I_{m p}\right)$ decreases as against the increase observed in the number of infected humans $\left(I_{m p}\right)$ in the uncontrolled case.

(c) Optimal control using: sleeping under mosquito treated nets $u_{1}(t)$, insecticide spray $u_{2}(t)$, vaccination $u_{4}(t)$, treatment for malaria $u_{5}(t)$, treatment for pneumonia $u_{6}(t)$, treatment for both malaria and pneumonia $u_{7}(t)$

Here, the control on sleeping under mosquito treated nets $u_{1}(t)$, insecticide spray $u_{2}(t)$, vaccination $u_{4}(t)$, treatment for malaria $u_{5}(t)$, treatment for pneumonia $u_{6}(t)$, treatment for both malaria and pneumonia $u_{7}(t)$ are used to optimize the objective function $J$ while sanitation $u_{3}(t)$ is set to zero. For this strategy, it was observed in Figure 33 that the number of infected humans with malaria-pneumonia $\left(I_{m p}\right)$ decreases as against the increase observed in the number of infected humans $\left(I_{m p}\right)$ in the uncontrolled case.

(d) Optimal control using: insecticide spray $u_{2}(t)$, sanitation $u_{3}(t)$, vaccination $u_{4}(t)$, treatment for malaria $u_{5}(t)$, treatment for pneumonia $u_{6}(t)$, treatment for both malaria and pneumonia $u_{7}(t)$

The control on sleeping under mosquito treated nets $u_{1}(t)$ is set to zero while the controls on insecticide spray $u_{2}(t)$, sanitation $u_{3}(t)$, vaccination $u_{4}(t)$, treatment for malaria $u_{5}(t)$, treatment for pneumonia $u_{6}(t)$, treatment for both malaria and pneumonia $u_{7}(t)$ are used to optimize the objective function $J$. Using this strategy, it was observed in Figure 34 that the number of infected humans with malaria-pneumonia $\left(I_{m p}\right)$ decreases as against the increase observed in the number of infected humans $\left(I_{m p}\right)$ in the uncontrolled case.

(e) Optimal control using: sanitation $u_{3}(t)$, vaccination $u_{4}(t)$, treatment for malaria $u_{5}(t)$, treatment for pneumonia $u_{6}(t)$, treatment for both malaria and pneumonia $u_{7}(t)$

For this strategy, the controls on sanitation $u_{3}(t)$, vaccination $u_{4}(t)$, treatment for malaria $u_{5}(t)$, treatment for pneumonia $u_{6}(t)$, treatment for both malaria and pneumonia $u_{7}(t)$ are used to optimize the objective function $J$ while the controls on sleeping under mosquito treated nets $u_{1}(t)$ and insecticide spray $u_{2}(t)$ are set to zero. It was observed as shown in Figure 35 that this strategy decreases the number of infected humans with malaria-pneumonia $\left(I_{m p}\right)$ while the number of infected humans with malaria-pneumonia $\left(I_{m p}\right)$ increases for the uncontrolled case.

(f) Optimal control using: insecticide spray $u_{2}(t)$, vaccination $u_{4}(t)$, treatment for malaria $u_{5}(t)$, treatment for pneumonia $u_{6}(t)$, treatment for both malaria and pneumonia $u_{7}(t)$

The control on insecticide spray $u_{2}(t)$, vaccination $u_{4}(t)$, treatment for malaria $u_{5}(t)$, treatment for pneumonia $u_{6}(t)$, treatment for both malaria and pneumonia $u_{7}(t)$ optimizes the objective function $J$ with the controls on sleeping under mosquito treated nets $u_{1}(t)$ and good sanitation $u_{3}(t)$ set to zero. For this control measure, it was shown in Figure 36 that the number of infected humans with malaria-pneumonia $\left(I_{m p}\right)$ reduces significantly from the uncontrolled case.

(g) Optimal control using: sleeping under mosquito treated nets $u_{1}(t)$, vaccination $u_{4}(t)$, treatment for malaria $u_{5}(t)$, treatment for pneumonia $u_{6}(t)$, treatment for both malaria and pneumonia $u_{7}(t)$

With this strategy, the control on sleeping under mosquito treated nets $u_{1}(t)$, vaccination $u_{4}(t)$, treatment for malaria $u_{5}(t)$, treatment for pneumonia $u_{6}(t)$, treatment for both malaria and pneumonia $u_{7}(t)$ are used to optimize the objective function $J$, while the control on insecticide spray $u_{2}(t)$ and sanitation $u_{3}(t)$ are all set to zero. The results in Figure 37 revealed a significant difference in the number of infected humans with malaria-pneumonia $\left(I_{m p}\right)$ compared to infected 
humans with malaria-pneumonia $\left(I_{m p}\right)$ without control. It was observed in Figure 37 that the control strategies resulted in a decrease in the number of infected humans $\left(I_{m p}\right)$ as against an increase in the uncontrolled case.

(h) Optimal control using: vaccination $u_{4}(t)$, treatment for malaria $u_{5}(t)$, treatment for pneumonia $u_{6}(t)$, treatment for both malaria and pneumonia $u_{7}(t)$

The objective function $J$ is optimized in this case using the control strategy on vaccination $u_{4}(t)$, treatment for malaria $u_{5}(t)$, treatment for pneumonia $u_{6}(t)$, treatment for both malaria and pneumonia $u_{7}(t)$, while the controls on sleeping under mosquito treated nets $u_{1}(t)$, insecticide spray $u_{2}(t)$ and sanitation $u_{3}(t)$ are all set to zero. We observed in Figure 38 that the control strategies resulted in a decrease in the number of infected humans with malaria-pneumonia $\left(I_{m p}\right)$. Similarly, From Figure 38, the uncontrolled case resulted in increased number of infected humans with malaria-pneumonia $\left(I_{m p}\right)$.

(i) Optimal control using: sanitation $u_{3}(t)$, treatment for malaria $u_{5}(t)$, treatment for pneumonia $u_{6}(t)$, treatment for both malaria and pneumonia $u_{7}(t)$

Using this strategy, the control on using sleeping under mosquito treated nets $u_{1}(t)$, insecticide spray $u_{2}(t)$ and vaccination $u_{4}(t)$ are all set to zero, while the control on sanitation $u_{3}(t)$, treatment for malaria $u_{5}(t)$, treatment for pneumonia $u_{6}(t)$, treatment for both malaria and pneumonia $u_{7}(t)$ are used to optimize the objective function $J$. The number of infected humans with malaria-pneumonia $\left(I_{m p}\right)$ was observed to be increasing for the uncontrolled case (dash dot red line) as shown in Figure 39 while the number of infected humans with malaria-pneumonia $\left(I_{m p}\right)$ decreases for the controlled case (solid green line).

(j) Optimal control using: insecticide spray $u_{2}(t)$, treatment for malaria $u_{5}(t)$, treatment for pneumonia $u_{6}(t)$, treatment for both malaria and pneumonia $u_{7}(t)$

The objective function $J$ here is optimized using insecticide spray $u_{2}(t)$, treatment for malaria $u_{5}(t)$, treatment for pneumonia $u_{6}(t)$, treatment for both malaria and pneumonia $u_{7}(t)$ as controls while the controls on sleeping under mosquito treated nets $u_{1}(t)$, sanitation $u_{3}(t)$ and vaccination $u_{4}(t)$ are all set to zero. It was observed in Figure 40 that this strategy resulted in significant decrease in the number of infected humans with malaria-pneumonia $\left(I_{m p}\right)$ while an increase was observed in the uncontrolled case.

(k) Optimal control using: sleeping under mosquito treated nets $u_{1}(t)$, treatment for malaria $u_{5}(t)$, treatment for pneumonia $u_{6}(t)$, treatment for both malaria and pneumonia $u_{7}(t)$

For this strategy, the control on insecticide spray $u_{2}(t)$, sanitation $u_{3}(t)$ and vaccination $u_{4}(t)$ are set to zero while the controls on sleeping under mosquito treated nets $u_{1}(t)$, treatment for malaria $u_{5}(t)$, treatment for pneumonia $u_{6}(t)$, treatment for both malaria and pneumonia $u_{7}(t)$ was used to optimize the objective function $J$. It was shown in Figure 41 that the controlled strategies resulted in a decrease in the number of infected humans with malaria-pneumonia $\left(I_{m p}\right)$ as against an increase observed in the uncontrolled case.

(l) Comparing all the control strategies

A comparison of all the control strategies for the malaria-pneumonia model case 3 was considered on infected humans with malaria-pneumonia $\left(I_{m p}\right)$ in Figure 42. It was observed that all the seven controls, followed by the combinations of six controls $\left(\left(u_{1}(t), u_{3}(t), u_{4}(t), u_{5}(t), u_{6}(t), u_{7}(t)\right)\right.$ and $\left(u_{2}(t), u_{3}(t), u_{4}(t), u_{5}(t), u_{6}(t), u_{7}(t)\right)$, five controls $\left(u_{3}(t), u_{4}(t), u_{5}(t), u_{6}(t), u_{7}(t)\right)$ and four controls $\left(u_{3}(t), u_{5}(t), u_{6}(t), u_{7}(t)\right)$ all led to a significant decrease in the number of infected humans with malaria-pneumonia $\left(I_{m p}\right)$. The strategies $\left(u_{1}(t), u_{5}(t), u_{6}(t), u_{7}(t)\right)$ and $\left(u_{2}(t), u_{5}(t), u_{6}(t), u_{7}(t)\right)$ yield a poorer result on the infected humans with malaria-pneumonia $\left(I_{m p}\right)$ because of poor or no sanitation. This result showed that with individuals sleeping under mosquito treated nets, spraying of insecticide in the sleeping area, vaccination and access to 
treatment may not be sufficient to control the spread of the disease in the community if poor or no sanitation is encouraged.

\subsection{Conclusion}

A nonlinear mathematical model has been developed and analyzed to study the dynamics of the co-infection of malaria-pneumonia using the mass action incidence. Here we incorporated the dynamics of sanitation by means of a linearly decreasing function both in terms of the transmission rate for malaria and contracting rate for pneumonia. The analysis of the model systems were performed using stability theory. Qualitative analysis of the model showed that the disease free equilibrium is locally asymptotically stable at threshold parameter less than unity and unstable at threshold greater than unity. Global stability of the model disease free equilibrium points were established using the comparison approach. Furthermore, the local stability of the model endemic equilibrium points were investigated by using Centre Manifold theory which showed that the malaria, pneumonia and the full malaria-pneumonia models endemic equilibriums are locally asymptotically stable whenever the associated basic reproduction number is greater than unity.

Sensitivity analysis of the model showed that the biting rate of mosquitoes, $\alpha$, contact rate $c$ and transmission rate $\beta_{p}$ of pneumonia are the most sensitive parameters in the present study, which suggest that more attention should be focused on these parameters. As a result, an optimal control strategy to curtail the spread of malaria, pneumonia and their co-infection was further studied in this work by incorporating treatments, sleeping under insecticide nets, spraying of insecticides and good sanitation as controls measures. The optimal analysis was carried out using the Pontryagin's Maximum Principle.

Finally, numerical results are provided to validate the analytical results.

\section{FUNDING}

This research receive no external funding

\section{CONFLICT OF INTEREST}

.The authors declare no conflict of interest.

\section{DATA AVAILABILITY STATEMENT}

The authors declare that all relevant data supporting the findings of this study are available within the article.

\section{APPENDIX A}

\section{Centre Manifold Theorem [9]}

Consider a general system of ODEs with a parameter $\boldsymbol{\phi}$

$\dot{x}=f(x, \phi) ; f: R^{n} \times R \rightarrow R^{n} ; f \in C^{2}\left(R^{n} \times R\right)$

Without loss of generality, assume that $x=0$ is equilibrium for system (A.1).

\section{Theorem A.1}

Assume:

(i) $A=D_{x} f(0,0)$ is the linearization matrix of system (2.1) around the equilibrium $x=0$ with $\phi$ evaluated at $O$. Zero is a simple eigenvalue of $A$ and all other eigenvalues of $A$ have negative real parts.

(ii) Matrix $A$ has a (nonnegative) right eigenvector $w$ and a left eigenvector $v$ corresponding 
to the zero eigenvalue. Let $f_{m}$ denote the $m$ th complements of

$$
f \text { and } a=\sum_{m, j, i=1}^{n} v_{m} w_{i} w_{j} \frac{\partial^{2} f_{m}(0,0)}{\partial x_{i} \partial x_{j}} ; \quad b=\sum_{m, j, i=1}^{n} v_{m} w_{i} \frac{\partial^{2} f_{m}(0,0)}{\partial x_{i} \partial \phi}
$$

Then the local dynamics of system (2.1) around $x=0$ are totally determined by $a$ and $b$

(iii) $a>0, b>0$. When $\phi<0$, with $|\phi| \ll 1, x=0$ is locally asymptotically stable and there exist a positive unstable equilibrium; when $0<\phi \ll 1, x=0$ is unstable and there exist a negative and locally asymptotically stable equilibrium

(iv) $a<0, b<0$. When $\phi<0$, with $|\phi| \ll 1, x=0$ is unstable; when $0<\phi \ll 1, x=0$ is locally asymptotically stable and there exist a positive unstable equilibrium.

(v) $a>0, b<0$. When $\phi<0$, with $|\phi| \ll 1, x=0$ is unstable and there exist a locally asymptotically stable negative equilibrium; when $0<\phi \ll 1, x=0$ is stable and a positive unstable equilibrium appears

(vi) $a<0, b>0$. When $\phi$ changes from negative to positive, $x=0$ changes its stability from stable to unstable. Correspondingly, a unstable equilibrium becomes positive and locally asymptotically stable.

The proof of theorem A.1 is found in [12].

\section{Pontryagin's Maximum Principle}

Theorem A.2: The necessary conditions that $\left(x_{0}{ }^{*}, u^{*}(t)\right)$ be an optimal initial condition and optimal control for the optimal control problem are the existence of a non-zero $k$-dimensional vector $\lambda$ with $\lambda_{1} \leq 0$ and an $n$-dimensional vector function $P(t)$ such that for $t \in\left[t_{0}, t_{1}\right]$ :

(i) $P(t)^{\prime}=-P(t)^{\prime} f_{x}\left(t, x^{*}(t), u^{*}(t)\right)$; for $t \in\left(t_{0}, t_{1}\right)$ and $u \in U$

(ii) $P(t)^{\prime}\left[f\left(t, x^{*}(t), u\right)-f\left(t, x^{*}(t), u^{*}(t)\right)\right] \leq 0$;

(iii) $P\left(t_{1}\right)^{\prime}=\lambda^{\prime} \phi_{x_{1}}(e)$;

(iv) $P\left(t_{0}\right)^{\prime}=-\lambda^{\prime} \phi_{x_{0}}(e)$;

(v) $P\left(t_{1}\right)^{\prime} f\left(t_{1}, x^{*}\left(t_{1}\right), u^{*}\left(t_{1}\right)\right)=-\lambda^{\prime} \phi_{t_{1}}(e)$;

(vi) $P\left(t_{0}\right)^{\prime} f\left(t_{0}, x^{*}\left(t_{0}\right), u^{*}\left(t_{0}\right)\right)=\lambda^{\prime} \phi_{t_{0}}(e)$;

If $f(t, x, u)$ has a continuous partial derivative $f_{t}(t, x, u)$, then the condition

(vii) $P(t)^{\prime} f\left(t, x^{*}(t), u^{*}(t)\right)=\lambda^{\prime} \phi_{t_{0}}\left(t_{0}, t_{1}, x^{*}\left(t_{0}\right), x^{*}\left(t_{1}\right)\right)+\int_{t_{0}}^{t} P(s)^{\prime} f_{t}\left(s, x^{*}(s), u^{*}(s)\right) d s$

holds for each $t \in\left[t_{0}, t_{1}\right]$.

The prove of theorem A.2 can be found in [15]

\section{REFERENCE}

[1] Adeniyi M.O (2018). "Optimal Control Model for the Transmission Dynamics of Malaria-Pneumonia Co-infection with Mass Action Incidence" Abstract submitted to 3rd International Conference on Infection, Disease Control and Prevention, June 25-26, 2018 Vancouver, Canada

[2] Agusto F. B, Marcus N, Okosun K. O. (2012). Application of optimal control to the epidemiology of malaria. Electronic Journal of Differential Equations, Vol. 2012, No. 81, pp. 1-22

[3] Allman E.S. and Rhodes J.A., (2004): Mathematical models in Biology an introduction. New York: Cambridge University press.

[4] Anderson Rm and May RM (1991). Infectious Diseases of Humans Dynamics and Control, Oxford University Press, Oxford

[5] Ariey F; Robert V (2003). The puzzling links between malaria transmission and drug 
resistance, Trends in Parasitology,19,158-160

[6] Arriola L., Hyman J, (2005): Lecture notes, forward and adjoint sensitivity analysis: with Application in Dynamical Systems, Linear Algebra and Optimization Mathematical and Theoretical Biology Institute, summer, 2005

[7] Blayneh K.W, Cao Y, Kwon H.D (2009)." Optimal control of vector-borne diseases: treatment and Prevention".DCDS Ser. B.;Vol. 11 , No. 3, pp587-611

[8] Brown, C. W, Kahoui, M. E. 1, Novotni, D. (2006); Weber, A.“Algorithmic methods for investigating equilibria in epidemic modelling", Journal of Symbolic Computation, 41, 1157-1173.

[9] Buonomo B. and Lacitignola D., (2011): On the backward bifurcation of a vaccination model with nonlinear incidence. Nonlinear Analysis: Modelling and Control, Vol.16, No.1, pp 30-46

[10] Burke A.C., (2010): Infectious diseases in Critical care Medicine. New York. Informal Health care U.S.A. Inc.

[11] Cai Li-Ming, Lashari Abid Ali, Jung II Hyo, Kazeem Oare Okosun and Young II Seo, (2013): Mathematical Analysis of Malaria Model with Partial Immunity to Reinfection. Journal of Abstract and Applied Analysis, Hindawi Publishing Corporation

[12] Chavez-Castillo and Song, B., (2004): Dynamical Models of Tuberculosis and their Applications. Mathematical Biosciences and Engineering, 1 (2): p p 361404.

[13] Chiyaka C, Tchuenche J.M, Garira W, Dube S (2008a).” A mathematical analysis of the effects of control strategies on the transmission dynamics of malaria". App. Math. Comput. Vol. 195, pp 641-662

[14] Collins W.E (2012). "Plasmodium knowlesi: A malaria parasite of monkeys and humans". Annual Review of Entomology Vol. 57, pp. 107-121

[15] Diekmann O, J.A.P Heesterbeek, (2000): Mathematical Epidemiology of Infectious Diseases. John Wiley 171 \& Son, Ltd

[16] Fleming W.H, Rishell R.W. Deterministic and Stochastic Optimal Control. Springer Verlag, New York, 1975.

[17] Gove S (1997). Integrated Management of childhood illness by outpatient health workers: technical basis and overview. Bull. WHO Vol. 75, (Supp 1), pp. 7-24

[18] Greenhalgh, D. (1992); Some results for an SEIR epidemic model with density dependent in the death rate. IMA, Journal Math. Appl. Med. Bio. Vol.9, pp. 67-106.

[19] Kallander K, Nsungwa-Sabiti J, Peterson S (2004). Symptoms overlap of malaria and Pneumonia-Policy implications for home management strategies. Acta Tropica Vol. 90, pp. 211-214

[20] Kermack, W. O.; McKendrick, A. G. (1991); Contributions to the Mathematical theory of epidemics- II. The Problem of endemicity. Bulletin of Mathematical Biolooy, Vol. 53, No. (1/2), pp. 57-87

[21] Kingsley N.U, Olufemi B.A, Ademola A.T (2011)." Clinical overlap between malaria and pneumonia: Can malaria rapid diagnostic test play a role?" Journal of Infection in Developing Countries. Vol. 5, No. 3, pp. 199-203

[22] Koram K.A, Molyneux M.E (2007), The Different Burden of Malaria Infection, Malaria Disease and Malaria-like Illnesses. Am. J. Trop.Med. Hyg., Vol. 77, Suppl. 6, pp. $1-5$

[23] Lakshmkantham V, Leela S. and Martynyuk A.A., (1989): Stability Analysis of Nonlinear Systems, Marcel Dekker, New York. ISBN 0-8247-8067-1. Pure and Applied Mathematics: A series of Monographs and Textbooks, Vol. 125.

[24] Lawi G.O, Mugisha J.Y.T and Omolo-Ongati N., (2013): Modelling Co-infection of 
Paediatric Malaria and Pneumonia. Int. Journal of Math. Analysis, Vol. 7, no. 9, pp. 413-424

[25] Mandell L.A., Wunderink R.G., Anzueto A. (2007): Infectious Diseases Society of America / American Thoracic Society Consensus Guidelines on the Management of Community Acquired Pneumonia in Adults. Clinical Infectious Diseases, 44: S27-S72

[26] McLuckie, A., (2009): Respiratory Disease and its Management. New York. Springer, pp: 51.

[27] Mushayabasa S., Tchuenche J.M.,Bhunu C.P., Ngarakana-Gwasira E., (2011): Modelling Gonorrhoea and HIV Co-interaction. Journal of Biological and Information Processing Sciences Vol. 103, pp. 27-37

[28] Okosun KO, Ouifki R, Marcus N (2011). Optimal control analysis of a malaria disease transmission model that includes treatment and vaccination with waning immunity. Biosystems Vol. 106, Issue 2-3, pp 136-145.

[29] Oluyo T.O. and Adeniyi M.O. (2014): Mathematical Analysis of Malaria-Pneumonia Model with Mass Action. International Journal of Applied Mathematics, ISSN: 2051-5227, Vol.29, Issue. 2

[30] Ranganathan, S.C; Sonnappa, S (February 2009). "Pneumonia and other respiratory infections". Pediatric clinics of North America Vol. 56, No. 1, pp. 135-56

[31] Rudan I, Boschi-Pinto C, Biloglav Z, Mulholland K, Campbell H (2008). Epidemiology and Etiology of childhood pneumonia. Bull.WHO Vol. 86, pp. 408-416

[32] Singh, V., Aneja, S., (2011): Pneumonia - Management in the Developing World. Paediatric Respiratory Reviews, Vol. 12, No. 1, pp. 52-59

[33] Snow R.W; Omumbo J (2006). Malaria, in Diseases and Mortality in Sub-Saharan Africa, D.T et al Jamison,ed.,The World Bank:195-213

[34] Tumwiine J., Mugisha J.Y.T and Luboobi L.S. (2007): Computational and Mathematical Methods in Medicine, Vol. 8, No. 3, 191-203

[35] Van den Driessche, P. and Watmough, J., (2002): Reproduction numbers and Subthreshold Endemic Equilibria for Compartmental models of Disease Transmission. Mathematical Bio-sciences, V o 1. 180, pp. 29-48

[36] WHO, (2009). Global Malaria Programme: Position Statement on Insecticide Treated Nets (ITNs).

[37] WHO, (2005). Technical Basis for the WHO Recommendations on the Management of Pneumonia in children at first-level health facilities. WHO/ARI/91-20

[38] WHO (2012): Pneumonia fact sheet

[39] Yang H. M (2001). A mathematical model for malaria transmission relating global warming and local socioeconomic conditions. Rev. Saude Publica, 35(3), 224-231.

[40] U.S. Bureau (2007), International database 